\title{
Two-dimensional metallicity distribution of the ionized gas in NGC 628 and NGC 6946*
}

\author{
B. Cedrés ${ }^{1,2}$, J. Cepa ${ }^{1,2}$, Á. Bongiovanni ${ }^{1,2}$, H. Castañeda ${ }^{3}$, M. Sánchez-Portal ${ }^{4}$, and A. Tomita ${ }^{5}$ \\ ${ }^{1}$ Instituto de Astrofísica de Canarias (IAC), 38200 La Laguna, Tenerife, Spain \\ e-mail: bce@iac.es \\ 2 Departamento de Astrofísica, Universidad de La Laguna (ULL), 38205 La Laguna, Tenerife, Spain \\ 3 Departamento de Física, Escuela Superior de Física y Matemáticas, IPN, 07738 México D.F., México \\ ${ }^{4}$ Herschel Science Centre, INSA/ESAC, 28691 Madrid, Spain \\ 5 Faculty of Education, Wakayama University, 640-8510 Wakayama, Japan
}

Received 9 May 2012 / Accepted 3 July 2012

\begin{abstract}
Aims. We present here two H II region catalogues with azimuthal resolution for the two grand design galaxies NGC 628 and NGC 6946. With the help of these catalogues, we study several properties of the star-forming processes occurring in spiral galaxies. Methods. We obtained direct imaging in the narrow-band filters centred at $\mathrm{H} \alpha, \mathrm{H} \beta$, [O II] $\lambda 3727$, and [O III] $\lambda \lambda 4959,5007$ and their respective continua. After the calibration and correction of the data, we obtained for each $\mathrm{HII}$ region the de-reddened fluxes in the aforementioned lines, the size, the $\mathrm{H} \alpha$ equivalent width, and, using two different empirical calibrations, the metallicity. Employing a method based on the Delaunay triangulation, a two-dimensional (2D) representation of the metallicity was obtained.

Results. Data for 209 H II regions of NGC 628 and 226 H II regions of NGC 6946 are obtained. The radial behaviours of the H $\alpha$ equivalent width, the excitation, and the oxygen abundance are derived. Two-dimensional representations of the metallicity and the excitation are calculated for the galaxies in the study. The two empirical calibrations of the metallicity are compared.

Conclusions. The behaviours of the extinction and the $\mathrm{H} \alpha$ equivalent width are similar to those presented in the literature. The oxygen abundance gradients obtained in this study agree with previously published values. However, more regions were examined than in previous studies. We find a difference of about 0.6 dex between the two empirical calibrations employed. Finally, the $2 \mathrm{D}$ representations of the metallicity reveal high metallicity knots in NGC 628, and for NGC 6946 a high metallicity azimuthal structure is discovered. These high metallicity regions seem to be linked to the arms of the galaxies and are probably produced by an increase in the temperature of the ionizing clusters in the H II regions, which may be linked to variations in the initial mass functions of the galaxies between the arm and interarm regions.
\end{abstract}

Key words. galaxies: spiral - HII regions - galaxies: abundances - galaxies: individual: NGC 628 - galaxies: individual: NGC 6946

\section{Introduction}

$\mathrm{H}$ II regions are excellent probes of the physical processes that drive star formation in the arms of grand design galaxies. It is therefore mandatory to determine the parameters that govern the H II state. Since the pioneering works of Searle (1971) and Hodge (1976), H II regions have been used to estimate the oxygen abundance in galaxies (e.g. Smith 1975; Rosa 1981; McCall et al. 1985; Kennicutt et al. 2003; Moustakas et al. 2010), to calculate the star formation rate (SFR) and star formation history, as proposed by Kennicutt (1998), or even variations in the initial mass function (IMF) associated with the presence of density waves (Cedrés et al. 2005).

Studies with data from H II regions in spiral galaxies at several wavelengths have indeed proven useful for the development of further studies (for example Hodge 1976; McCall et al. 1985; Belley \& Roy 1992; Cedrés \& Cepa 2002; Rozas 2008; Moustakas et al. 2010). Moreover, imaging studies give twodimensional (2D) information that is absent in long-slit works, and that may be important in order to understand the physical

* Full Tables 4-9 are available in electronic form at http://www. aanda.org processes that take place in the galaxy (Cedrés et al. 2005; Rosales-Ortega et al. 2011; Cedrés et al., in prep.).

The grand design galaxy NGC 628, with arm class 9 according to the Elmegreen \& Elmegreen (1987) classification scheme, has a low inclination (6.5 degrees, according to Kamphuis \& Briggs 1992) and a large number of bright H II regions (Table 1). It has been a principal target for the study of H II regions since the early works of Hodge (1976) and Kennicutt \& Hodge $(1976,1980)$. It was first reported to have an abundance gradient by Talent (1983). Other spectroscopic works that include this galaxy are McCall et al. (1985), Ferguson et al. (1998), Bresolin et al. (1999), and Castellanos et al. (2002) and others. The first narrow-band study of this galaxy was done by Belley \& Roy (1992; hereafter B\&R92), who obtained data for up to $130 \mathrm{H}$ II regions in several emission lines, including $\mathrm{H} \alpha, \mathrm{H} \beta$, [OIII], and [NII]. A more detailed study, using integral field spectroscopy, for a number of emission lines was carried out by Sánchez et al. (2011) and Rosales-Ortega et al. (2011; hereafter RO11) with data for $108 \mathrm{H}$ II regions. They were also the first to do a proper $2 \mathrm{D}$ spectroscopic study of the oxygen abundance in a spiral galaxy.

The galaxy NGC 6946 is also a grand design spiral of the same arm class as NGC 628. However, at optical wavelenghts and in the $K$-band (Regan \& Vogel 1995), NGC 6946 has a more 
Table 1. Parameters of the galaxy sample.

\begin{tabular}{lcccccc}
\hline \hline Galaxy & Morphological class & $D(\mathrm{Mpc})$ & $\rho_{25}(\operatorname{arcmin})$ & $V\left(\mathrm{~km} \mathrm{~s}^{-1}\right)$ & PA (degrees) & $i$ (degrees) \\
\hline NGC 628 & SA(s)c (1) & $9.7(2)$ & $5.2 \pm 0.1(1)$ & $657 \pm 1(3)$ & $12 \pm 1(4)$ & $6.5(5)$ \\
NGC 6946 & SAB(rs)cd (1) & $5.5(2)$ & $5.7 \pm 0.1(1)$ & $40 \pm 2(6)$ & $64(7)$ & $30(7)$ \\
\hline
\end{tabular}

Notes. (1) de Vaucouleurs et al. (1991); (2) Tully. (1988); (3) Lu et al. (1993); (4) Egusa et al. (2009); (5) Kamphuis \& Briggs (1992); (6) Epinat et al (2008); (7) Sofue et al. (1999).

Table 2. Employed filters.

\begin{tabular}{lccc}
\hline \hline Line & $\lambda_{\mathrm{c}}(\AA)$ & $F W H M(\AA)$ & Maximun transmitance $(\%)$ \\
\hline$[\mathrm{O}$ II] continuum & 3578 & 25 & 48 \\
{$[$ O II] } & 3728 & 32 & 27 \\
H $\beta$ continuum (Str b) & 4670 & 180 & 89 \\
H $\beta$ & 4873 & 45 & 58 \\
{$[\mathrm{O}$ III] } & 5010 & 43 & 56 \\
{$[\mathrm{O} \mathrm{III}]$ continuum } & 5105 & 37 & 55 \\
H $\alpha$ & 6577 & 180 & 77 \\
H $\alpha$ continuum & 6788 & 45 & 73 \\
\hline
\end{tabular}

Table 3. Total integration times at each line and continuum.

\begin{tabular}{lcccccccc}
\hline \hline & $\mathrm{H} \alpha$ & $\mathrm{H} \alpha_{\mathrm{c}}$ & $\mathrm{H} \beta$ & $\mathrm{H} \beta_{\mathrm{c}}$ & {$[\mathrm{OII}]$} & {$[\mathrm{OII}]_{\mathrm{c}}$} & {$[\mathrm{OIII}]$} & {$[\mathrm{OIII}]_{\mathrm{c}}$} \\
\hline NGC 628 & $3 \times 600 \mathrm{~s}$ & $5 \times 600 \mathrm{~s}$ & $3 \times 1200 \mathrm{~s}$ & $3 \times 600 \mathrm{~s}$ & $4 \times 900 \mathrm{~s}$ & $4 \times 1200 \mathrm{~s}$ & $3 \times 900 \mathrm{~s}$ & $3 \times 900 \mathrm{~s}$ \\
NGC 6946 & $3 \times 600 \mathrm{~s}$ & $3 \times 600 \mathrm{~s}$ & $3 \times 1200 \mathrm{~s}$ & $3 \times 600 \mathrm{~s}$ & $3 \times 900 \mathrm{~s}$ & $3 \times 1200 \mathrm{~s}$ & $3 \times 900 \mathrm{~s}$ & $3 \times 900 \mathrm{~s}$ \\
\hline
\end{tabular}

complex shape with multiple arms, which leads us to suggest that it is more flocculent than NGC 628 (Foyle et al. 2010). It has a higher inclination (30 degrees from Sofue et al. 1999) than NGC 628 and a large number of bright H II regions (Table 1). This galaxy has also been a primary target for cataloguing $\mathrm{H}$ II regions in spiral galaxies in the studies of McCall et al. (1985), Ferguson et al. (1998), B\&R92, and Moustakas et al. (2010), among others. Unfortunately, for this galaxy there are fewer studies at high spectroscopic resolution. That of GarcíaBenito et al. (2010) is the most noteworthy, but unfortunately only covers a few H II regions in the outer parts of the galaxy.

In this article, we present the data collected using narrowband imaging techniques for NGC 628 and NGC 6946 in the emission lines $\mathrm{H} \alpha, \mathrm{H} \beta,[\mathrm{O}$ II] $\lambda 3727 \AA$, and [O III] $\lambda 5007 \AA$ and their respective continua. In Sect. 2, we describe how we obtained, reduced, and corrected the data. In Sect. 3, we present the resultant $\mathrm{H}$ II catalogue and explore the results for several parameters, such as the $\mathrm{H} \alpha$ equivalent width, the extinction, and the sizes of the H II regions. In Sect. 4, we deal with the problem of the determination of the oxygen abundance for both galaxies, and we present a 2D model of the metallicity. In Sect. 5, a summary is presented.

\section{Data}

The data were obtained in an observing run during the period 2005 September 24-26 (inclusive) at the $2.5 \mathrm{~m}$ Nordic Optical Telescope (NOT), located at Roque de los Muchachos Observatory. The instrument used was ALFOSC in direct imaging mode with narrow-band filters centred on the lines $[\mathrm{O}$ II $] \lambda 3727 \AA$, [O III] $\lambda 5007 \AA, \mathrm{H} \alpha$, and $\mathrm{H} \beta$ and their respective continua. The filters used are described in Table 2. The spatial scale given by NOT with ALFOSC and E2V CCD $\left(2048 \times 2048\right.$ pixels) was $0.19^{\prime \prime} /$ pixel. The seeing was between $0.9^{\prime \prime}$ and $1^{\prime \prime}$. The integration times for both galaxies in each filter are indicated in Table 3.

\subsection{Reduction and calibration}

The reduction and calibration of the data were carried out using the IRAF package ${ }^{1}$. The images were bias-subtracted, employing bias frames taken during the night and the overscan zone of each image. The images were then flatfield-corrected using sky flatfields (three or four for each filter), taken during twilight at the beginning and end of the observing nights. To correct the images for sky emission, we obtained the mean value in $20 \times 20$ pixel boxes in zones unaffected by the galaxy, bright stars, or cosmic rays.

The images were calibrated using spectrophotometric standard stars from Oke \& Gunn (1983) and Oke (1990), and following the procedure described by Barth et al. (1994).

After the calibration, we matched and combined all the images of the galaxy in the same filter using the median. In this way, we were able to remove the cosmic-ray hits and increase the signal to noise of the final images.

The continuum was subtracted from each line image using the method described in Cedrés \& Cepa (2002). The calibrated and continuum-subtracted images for NGC 628 and NGC 6946 in all the lines are presented in Figs. 1 and 2, respectively.

\subsection{Flux extraction}

The H II regions were detected and their fluxes were extracted by employing the FOCAS task. To detect each region, we used the image with the highest signal to noise (the $\mathrm{H} \alpha$ image) and

\footnotetext{
${ }^{1}$ IRAF is distributed by the National Optical Astronomy Observatory, which is operated by the Association of Universities for Research in Astronomy (AURA) under cooperative agreement with the National Science Foundation.
} 

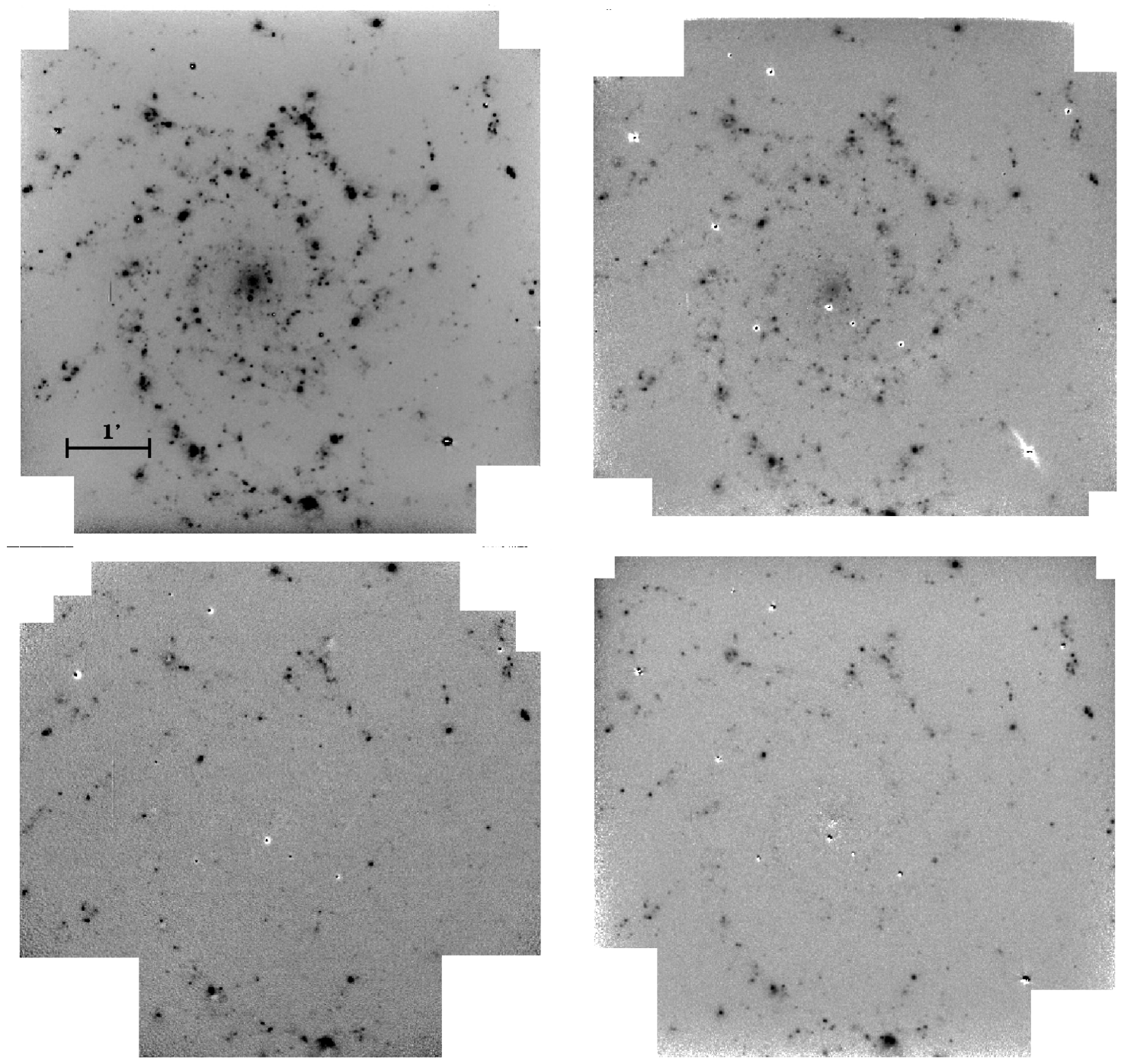

Fig. 1. Continuum-subtracted images for NGC 628. Top left: $\mathrm{H} \alpha$. Top right: $\mathrm{H} \beta$. Bottom left: [OII]. Bottom right: [OIII]. North is at top and east to the left for all images. Scale as indicated.

assumed a region was detected when it had a mean of $3 \sigma$ flux per pixel above background. Following the method described in Cedrés \& Cepa (2002), a limiting isophote was then created for each region with a flux of $1 \sigma$ per pixel above the background. With those isophotes, we were able to obtain the fluxes in all the lines within the defined area in $\mathrm{H} \alpha$. If a region in one of the lines considered had a flux below the $3 \sigma$ per pixel limit, the flux of that region in the corresponding line was discarded. In total, we detected 209, 101, and $94 \mathrm{H}$ II regions for NGC 628 in the $\mathrm{H} \alpha(\mathrm{H} \beta),[\mathrm{OII}]$, and [OIII] lines respectively, and 226, 85, and $80 \mathrm{H}$ II regions in $\mathrm{H} \alpha(\mathrm{H} \beta)$, [OII], and [OIII] lines respectively, for NGC 6946.

\subsection{Corrections applied to the data}

To correct the emission lines for Galactic and extragalactic extinction, we obtained first the $A_{\mathrm{V}}$ value using the ratios of the $\mathrm{H} \alpha$ and $\mathrm{H} \beta$ lines, assuming case $\mathrm{B}$ recombination, an electron density of $100 \mathrm{~cm}^{-3}$, a temperature of $10000 \mathrm{~K}$ (James \& Puxley 1993) and the Seaton (1979) extinction law. Once we obtained $A_{\mathrm{V}}$, employing the reddening law given by Schild (1977), we were able to determine the extinction for all the lines considered.

The underlying absorption due to massive stars in H II regions and the emission from the underlying galactic disc in the
$\mathrm{H} \alpha$ and $\mathrm{H} \beta$ continua were corrected for, using the methods described in Cedrés \& Cepa (2002).

The $\mathrm{H} \alpha$ fluxes were also corrected for the contribution of [NII] $\lambda \lambda 6548,6584$ lines using data from McCall et al. (1985).

To calculate the total flux in [OIII] $\lambda \lambda 44959,5007$, we assumed the theoretical relation $I([\mathrm{OIII}] \lambda \lambda 4959,5007)=$ 1.34 I([OIII $] \lambda 5007)$.

\section{Results}

The physical parameters of the detected H II regions studied for both galaxies are summarized in Tables 4 to 7 for the first ten regions of each galaxy.

In Fig. 3, the relative positions of the detected $\mathrm{H}$ II regions for NGC 628 and NGC 6946, and also the positions of the arms, as determined in Cedrés et al. (in prep.), are shown.

In Fig. 4, we have represented the histograms of the area (in square kpc) of the detected regions. For NGC 6946, there are more regions with an area smaller than $10 \mathrm{kpc}^{2}$ than for NGC 628. This is unsurprising, because NGC 6946 is closer to us than NGC 628 and the seeing for both observations is similar; we are therefore able to resolve smaller regions in images with the same depth for both galaxies. 

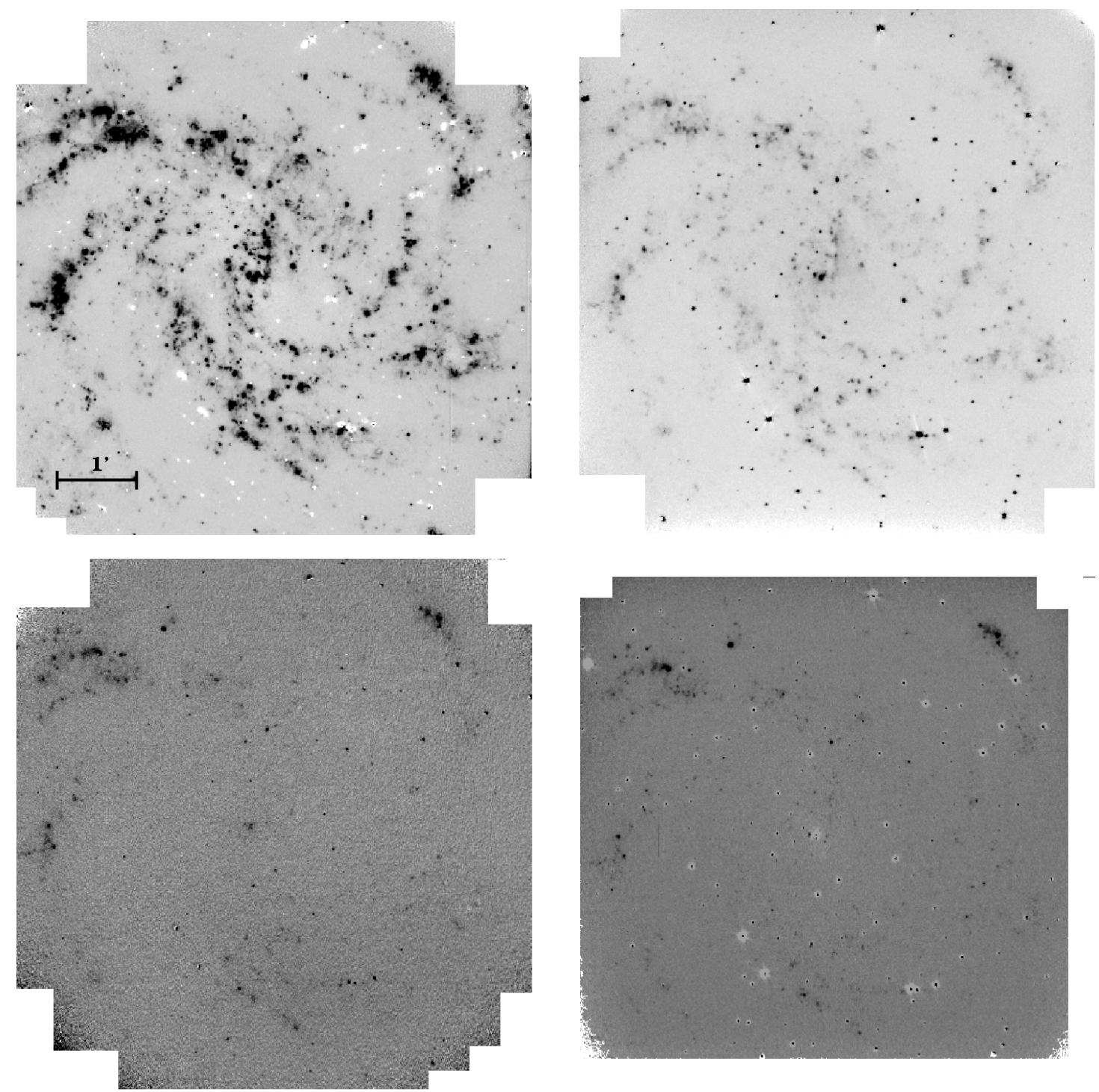

Fig. 2. Continuum-subtracted images for NGC 6946. Top left: $\mathrm{H} \alpha$. Top right: $\mathrm{H} \beta$. Bottom left: [OII]. Bottom right: [OIII]. North is at the top and east to the left for all images. Scale as indicated.

The extinction derived (represented as histograms in Fig. 5) for both galaxies differs somewhat from the values obtained by B\&R92. Our extinction is higher for NGC 628, with the maximum in the bin centred at $A_{\mathrm{V}}=0.9$ (with a mean value $A_{\mathrm{V}}=0.79$ ). The maximum of $\mathrm{B} \& \mathrm{R} 92$ is at the bin centred at $A_{\mathrm{V}}=0.125$ (with mean value $A_{\mathrm{V}}=0.52$ ). However, Sánchez et al. (2011) give a mean value for $A_{\mathrm{V}}$ between 1.24 and 1.04, depending on the method employed (H II regions or integrated spectrum, respectively); this is closer to our results. Moreover, Sánchez et al. (2011) also found, like us, extinctions higher than $A_{\mathrm{V}}=2.5$, while the higher extinction found in B\&R92 is about $A_{\mathrm{V}} \simeq 1.6$. For NGC 6946, the derived value of the extinction for $\operatorname{B} \& \operatorname{R} 92\left(A_{\mathrm{V}}=1.01\right)$ is slighty higher than ours $\left(A_{\mathrm{V}}=0.9\right)$; however, the distribution and range of the extinction values are similar for both datasets.

The histograms for the logarithm of the $\mathrm{H} \alpha$ equivalent width for NGC 628 (left) and NGC 6946 (right) are presented in Fig. 6. The mean value of the $\mathrm{H} \alpha$ equivalent width is slightly greater for NGC 6946 at $\log (\mathrm{EWH} \alpha)=2.6$ than for NGC 628 is $\log (\mathrm{EWH} \alpha)=2.28$. As discussed in Cedrés et al. (in prep.), this may be equivalent to mean older H II regions for NGC 628, if we assumed a Salpeter IMF for both galaxies. From Fig. 7, where we represent the logarithm of the $\mathrm{H} \alpha$ equivalent width as a function of the galactocentric radius for both galaxies, there is no clear trend of the equivalent width with radius for the galaxies in the sample. A similar result was obtained in Cedrés \& Cepa (2002) for the galaxies NGC 5457 and NGC 4395.

In Fig. 8, we have presented the logarithm of the [OIII] $\lambda \lambda 4959,5007 / \mathrm{H} \beta$ ratio as a function of galactocentric radius. When comparing both galaxies with data from the literature, there is a clear agreement with our results, even for data obtained using different techniques, such as the RO11 data for NGC 628. The $[\mathrm{OIII}] / \mathrm{H} \beta$ ratio increases with galactocentric distances for both galaxies, which indicates that there is a nonzero oxygen abundance gradient in both galaxies. The data from B\&R92 covers a slightly larger field for both galaxies than our data, so more regions are presented over $R / R_{25}>0.75$, and these are the ones with higher mean values for [OIII] $\lambda \lambda 4959$, $5007 / \mathrm{H} \beta$.

The relationship between the logarithm of the $[\mathrm{OII}] / \mathrm{H} \beta$ and $[\mathrm{OIII}] / \mathrm{H} \beta$ ratios is presented in Fig. 9 for NGC 628 and NGC 6946. For both galaxies, the data are distributed in the expected locus, and for NGC 628 there is very good agreement with RO11 data. However, for NGC 6946, the [OIII]/H $\beta$ ratio 
B. Cedrés et al.: Two-dimensional metallicity distribution in NGC 628 and NGC 6946

Table 4. Positions and main properties of the first ten H II regions of NGC 628. The H $\alpha$ fluxes are extinction corrected.

\begin{tabular}{|c|c|c|c|c|c|c|}
\hline Number & $X$ offset $(\operatorname{arcsec})$ & $Y$ offset $(\operatorname{arcsec})$ & Deprojected $R$ (arcmin) & $F_{\mathrm{H} \alpha}\left(10^{14} \mathrm{erg} / \mathrm{cm}^{2} / \mathrm{s}\right)$ & $A_{\mathrm{V}}(\mathrm{mag})$ & Area $\left(\operatorname{arcsec}^{2}\right)$ \\
\hline 1 & 87.49 & 183.08 & 3.39 & $14.78 \pm 1.45$ & $0.00 \pm 0.06$ & 36.2 \\
\hline 2 & 3.32 & 181.94 & 3.03 & $6.25 \pm 0.64$ & $0.00 \pm 0.06$ & 37.1 \\
\hline 3 & 14.15 & 176.81 & 2.96 & $1.55 \pm 0.18$ & $0.00 \pm 0.07$ & 9.9 \\
\hline 4 & 71.82 & 171.11 & 3.10 & $0.41 \pm 0.06$ & $0.00 \pm 0.09$ & 3.6 \\
\hline 5 & -43.51 & 153.92 & 2.67 & $7.86 \pm 0.79$ & $0.00 \pm 0.06$ & 18.6 \\
\hline 6 & 69.82 & 151.92 & 2.79 & $0.41 \pm 0.04$ & $0.79 \pm 0.11$ & 2.8 \\
\hline 7 & 167.96 & 145.75 & 3.72 & $1.61 \pm 0.18$ & $0.07 \pm 0.07$ & 8.3 \\
\hline 8 & 166.15 & 142.23 & 3.66 & $0.63 \pm 0.08$ & $0.15 \pm 0.09$ & 4.5 \\
\hline 9 & 145.25 & 138.15 & 3.36 & $1.16 \pm 0.14$ & $0.00 \pm 0.08$ & 8.4 \\
\hline 10 & 165.77 & 136.34 & 3.60 & $0.87 \pm 0.10$ & $0.32 \pm 0.08$ & 6.5 \\
\hline
\end{tabular}

Notes. The complete table is available electronically.

Table 5. Positions and main properties of the first ten H II regions of NGC 6946. The H $\alpha$ fluxes are extinction corrected.

\begin{tabular}{|c|c|c|c|c|c|c|}
\hline Number & $X$ offset $(\operatorname{arcsec})$ & $Y$ offset $(\operatorname{arcsec})$ & Deprojected $R$ (arcmin) & $F_{\mathrm{H} \alpha}\left(10^{14} \mathrm{erg} / \mathrm{cm}^{2} / \mathrm{s}\right)$ & $A_{\mathrm{V}}(\mathrm{mag})$ & Area $\left(\operatorname{arcsec}^{2}\right)$ \\
\hline 1 & 67.36 & 187.27 & 3.82 & $0.57 \pm 0.08$ & $0.09 \pm 0.09$ & 4.9 \\
\hline 2 & 111.44 & 171.78 & 3.93 & $0.60 \pm 0.07$ & $0.52 \pm 0.10$ & 5.3 \\
\hline 3 & 127.02 & 145.18 & 3.68 & $69.81 \pm 4.17$ & $0.66 \pm 0.06$ & 196.6 \\
\hline 4 & -59.09 & 154.11 & 2.96 & $0.68 \pm 0.06$ & $0.97 \pm 0.10$ & 3.6 \\
\hline 5 & 114.86 & 151.55 & 3.64 & $0.72 \pm 0.06$ & $1.14 \pm 0.11$ & 3.2 \\
\hline 6 & 66.69 & 147.65 & 3.12 & $2.68 \pm 0.15$ & $1.31 \pm 0.08$ & 8.8 \\
\hline 7 & 36.96 & 146.89 & 2.90 & $3.43 \pm 0.21$ & $1.01 \pm 0.08$ & 13.1 \\
\hline 8 & -56.33 & 144.33 & 2.77 & $2.99 \pm 0.28$ & $0.34 \pm 0.07$ & 20.2 \\
\hline 9 & 116.47 & 143.76 & 3.54 & $1.30 \pm 0.09$ & $1.16 \pm 0.09$ & 6.5 \\
\hline 10 & -62.79 & 138.72 & 2.70 & $6.21 \pm 0.33$ & $1.12 \pm 0.07$ & 10.2 \\
\hline
\end{tabular}

Notes. The complete table is available electronically.

Table 6. Equivalent width, $\mathrm{H} \beta$ flux and, oxygen to $\mathrm{H} \beta$ flux ratios, both extinction corrected, for the first ten $\mathrm{H}$ II regions of NGC 628 .

\begin{tabular}{lcccc}
\hline \hline Number & $\log (\mathrm{EWH} \alpha)(\AA)$ & $F_{\mathrm{H} \beta}\left(10^{14} \mathrm{erg} / \mathrm{cm}^{2} / \mathrm{s}\right)$ & $\log ([\mathrm{OII}] / \mathrm{H} \beta)$ & $\log ([\mathrm{OIII}] / \mathrm{H} \beta)$ \\
\hline 1 & $2.50 \pm 0.07$ & $6.04 \pm 1.35$ & $0.50 \pm 0.10$ & $0.26 \pm 0.10$ \\
2 & $2.34 \pm 0.08$ & $3.03 \pm 0.71$ & $0.47 \pm 0.11$ & $0.08 \pm 0.10$ \\
3 & $2.52 \pm 0.09$ & $0.64 \pm 0.17$ & $0.48 \pm 0.14$ & $0.12 \pm 0.12$ \\
4 & $2.83 \pm 0.11$ & $0.18 \pm 0.06$ & - & - \\
5 & $0.53 \pm 0.07$ & $4.80 \pm 0.99$ & - & - \\
6 & $2.34 \pm 0.08$ & $0.14 \pm 0.03$ & - & - \\
7 & $2.69 \pm 0.09$ & $0.56 \pm 0.14$ & $0.38 \pm 0.14$ & $0.22 \pm 0.12$ \\
8 & $2.53 \pm 0.10$ & $0.22 \pm 0.06$ & - & - \\
9 & $2.25 \pm 0.09$ & $0.52 \pm 0.14$ & $0.31 \pm 0.16$ & $0.19 \pm 0.13$ \\
10 & $1.83 \pm 0.08$ & $0.30 \pm 0.07$ & - & - \\
\hline
\end{tabular}

Notes. The complete table is available electronically.

Table 7. Equivalent width, $\mathrm{H} \beta$ flux and, oxygen to $\mathrm{H} \beta$ flux ratios, both extinction corrected, for the first ten $\mathrm{H}$ II regions of NGC 6946.

\begin{tabular}{lcccc}
\hline \hline Number & $\log (\mathrm{EWH} \alpha)(\AA)$ & $F_{\mathrm{H} \beta}\left(10^{14} \mathrm{erg} / \mathrm{cm}^{2} / \mathrm{s}\right)$ & $\log ([\mathrm{OII}] / \mathrm{H} \beta)$ & $\log ([\mathrm{OIII}] / \mathrm{H} \beta)$ \\
\hline 1 & $2.97 \pm 0.13$ & $0.20 \pm 0.06$ & - & - \\
2 & $2.86 \pm 0.10$ & $0.21 \pm 0.04$ & $0.53 \pm 0.09$ & $-0.43 \pm 0.09$ \\
3 & $2.69 \pm 0.05$ & $24.05 \pm 2.80$ & $0.45 \pm 0.05$ & $-0.09 \pm 0.05$ \\
4 & $2.59 \pm 0.08$ & $0.23 \pm 0.03$ & - & - \\
5 & $3.25 \pm 0.08$ & $0.25 \pm 0.03$ & $0.53 \pm 0.06$ & $-0.48 \pm 0.06$ \\
6 & $3.11 \pm 0.05$ & $0.90 \pm 0.08$ & $0.49 \pm 0.04$ & $-0.01 \pm 0.04$ \\
7 & $2.88 \pm 0.06$ & $1.17 \pm 0.13$ & $0.38 \pm 0.05$ & $-0.56 \pm 0.05$ \\
8 & $2.54 \pm 0.08$ & $1.04 \pm 0.20$ & $0.34 \pm 0.08$ & $-0.52 \pm 0.08$ \\
9 & $2.92 \pm 0.07$ & $0.44 \pm 0.05$ & $0.50 \pm 0.05$ & $-0.61 \pm 0.05$ \\
10 & $2.89 \pm 0.05$ & $2.11 \pm 0.20$ & - & - \\
\hline
\end{tabular}

Notes. The complete table is available electronically. 

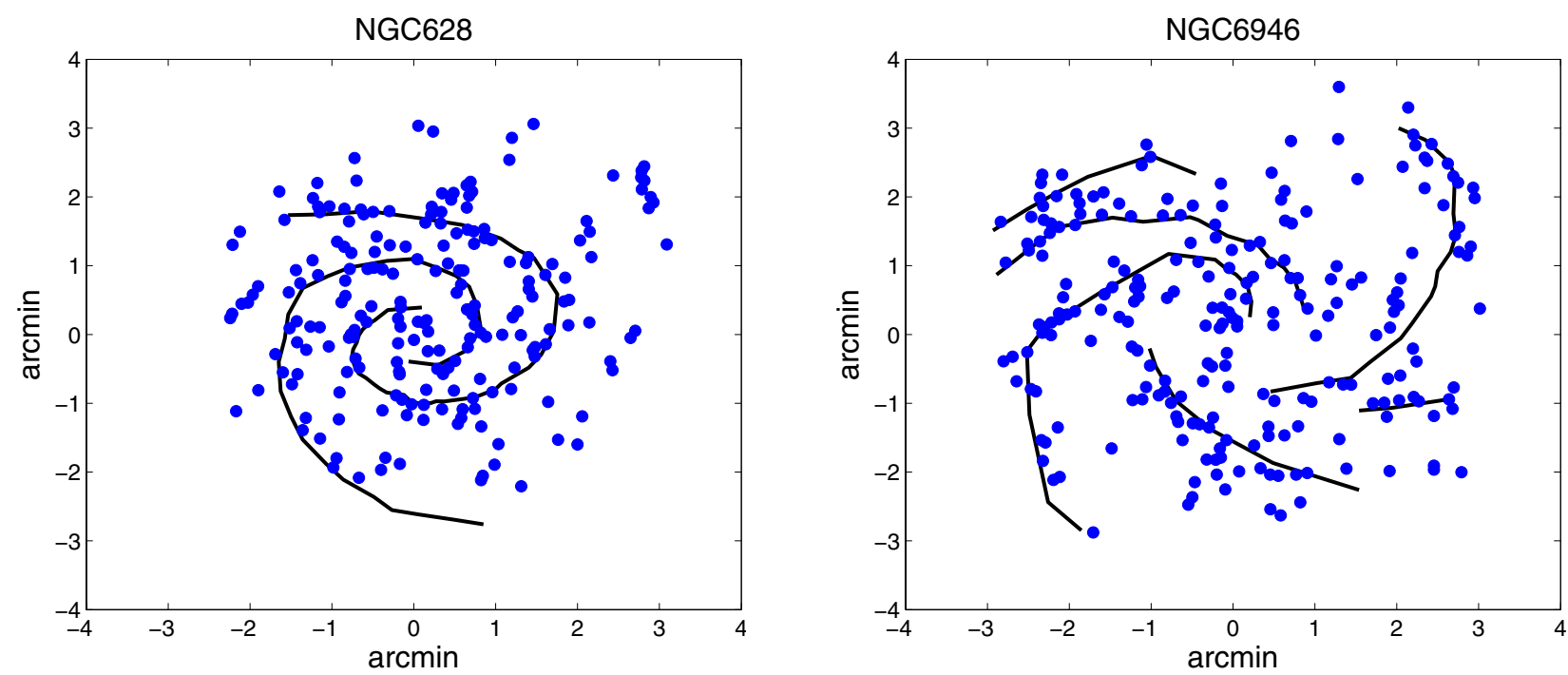

Fig. 3. Positions of the H II regions detected for NGC 628 (left panel) and NGC 6946 (right panel). The arms, as determined in Cedrés et al. (in prep.), are indicated by the continuous lines. North is at the top and east to the left.
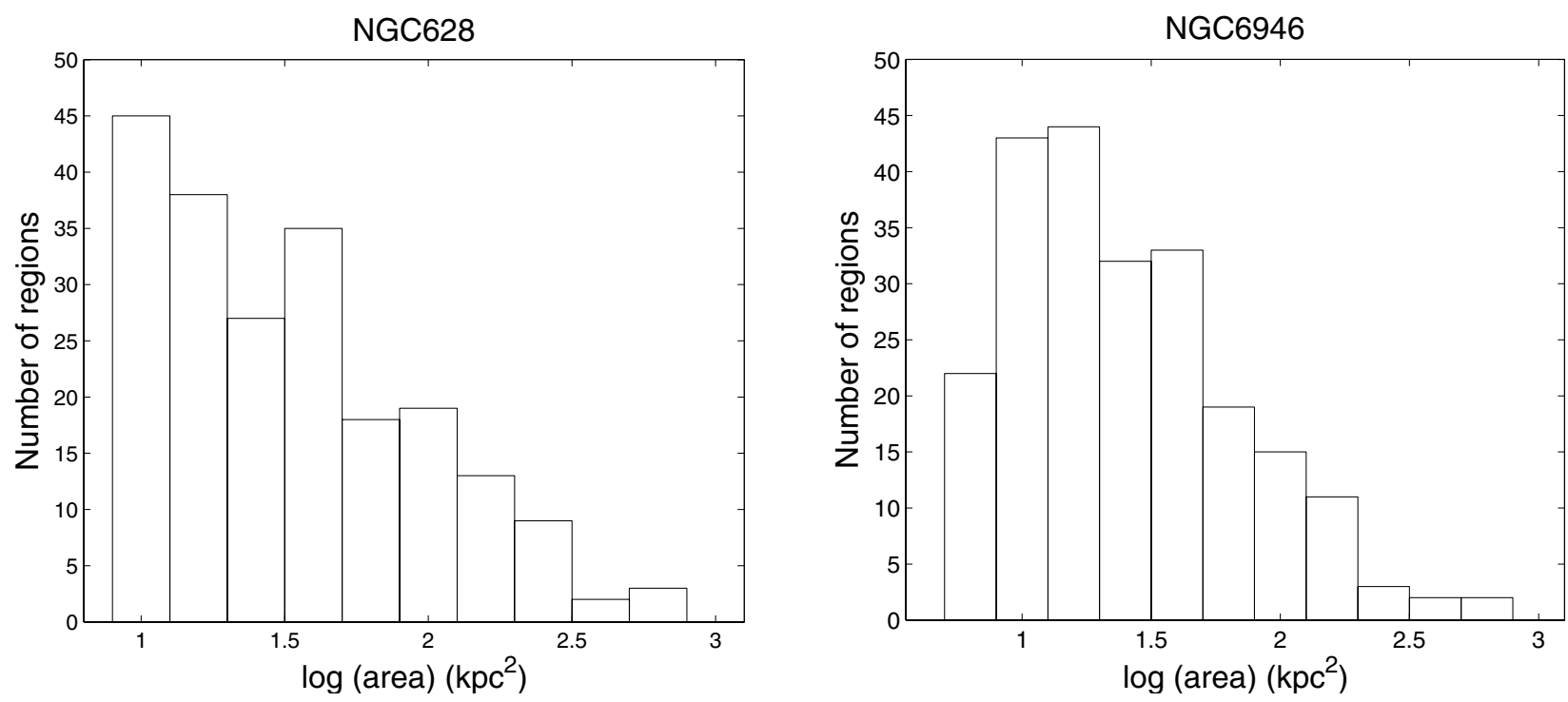

Fig. 4. Histograms of the areas of the H II regions in $\mathrm{kpc}^{2}$, for NGC 628 (left panel) and NGC 6946 (right panel).
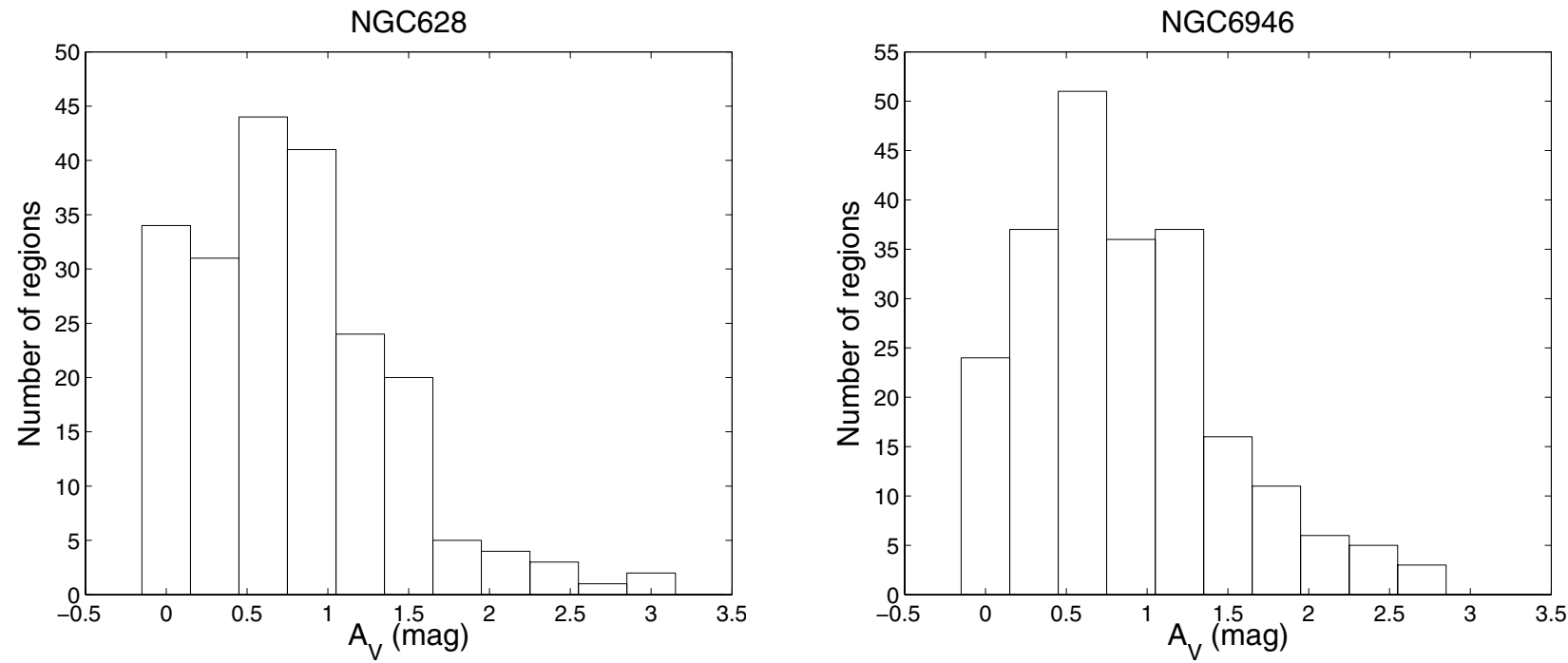

Fig. 5. Histograms of the extinctions of the H II regions in magnitudes for NGC 628 (left panel) and NGC 6946 (right panel). 
B. Cedrés et al.: Two-dimensional metallicity distribution in NGC 628 and NGC 6946
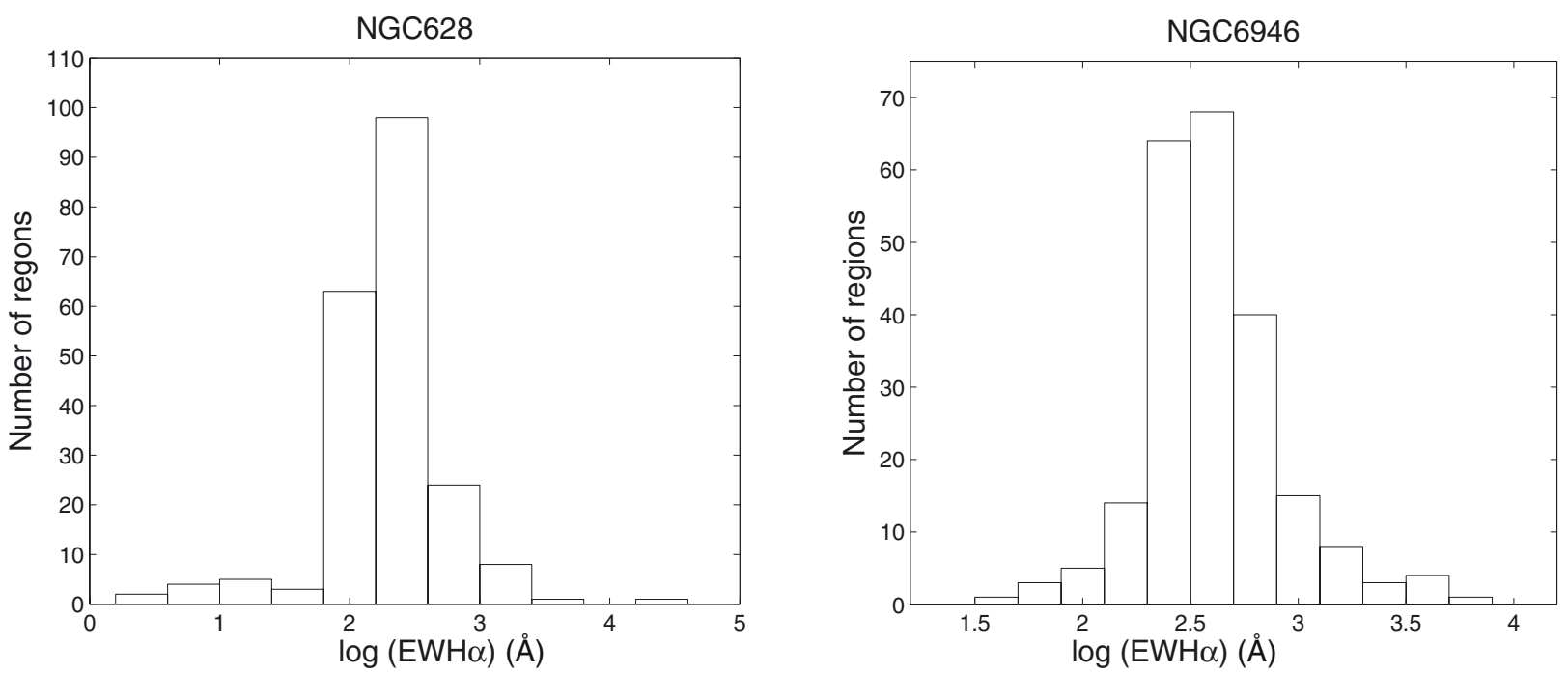

Fig. 6. Histograms of the logarithm of the H $\alpha$ equivalent width for the H II regions (in $\AA$ ) for NGC 628 (left panel) and NGC 6946 (right panel).
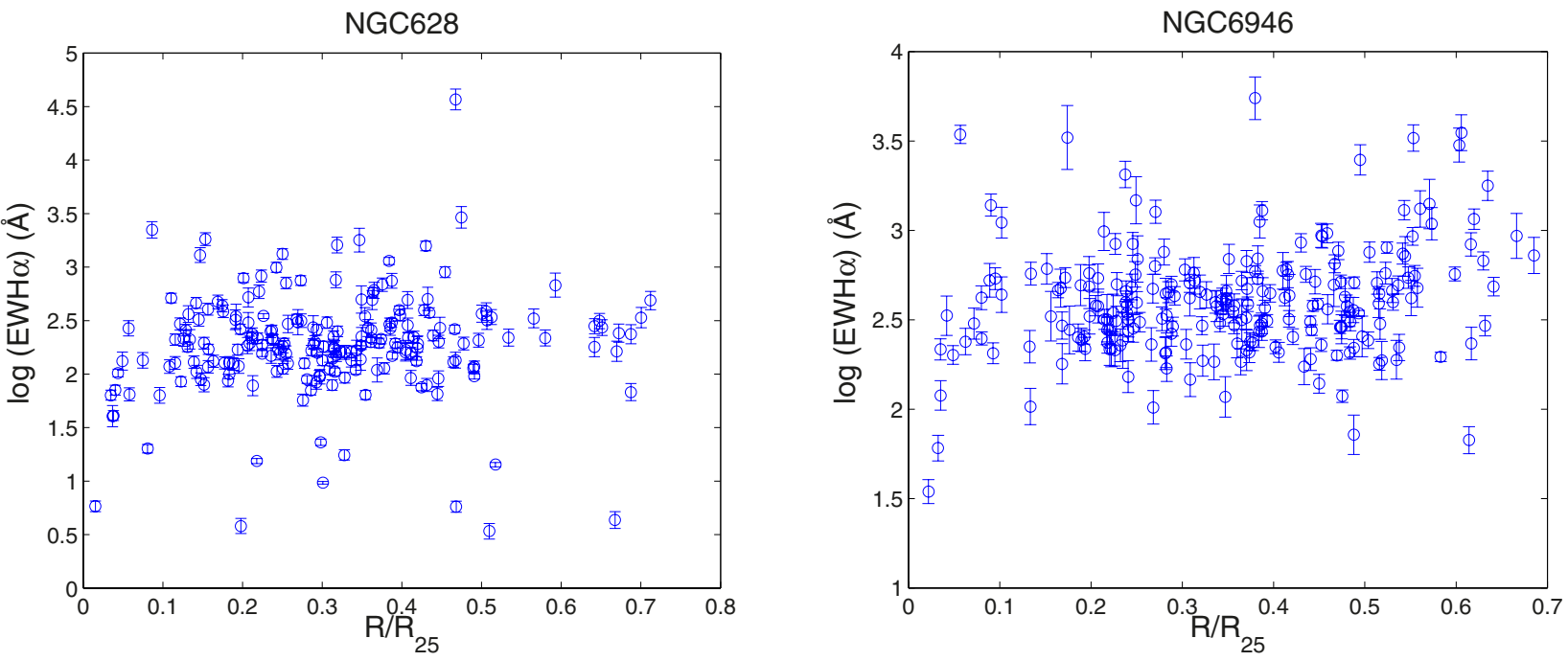

Fig. 7. Logarithm of the H $\alpha$ equivalent width as a function of the $R_{25}$ radius for NGC 628 (left panel) and NGC 6946 (right panel).
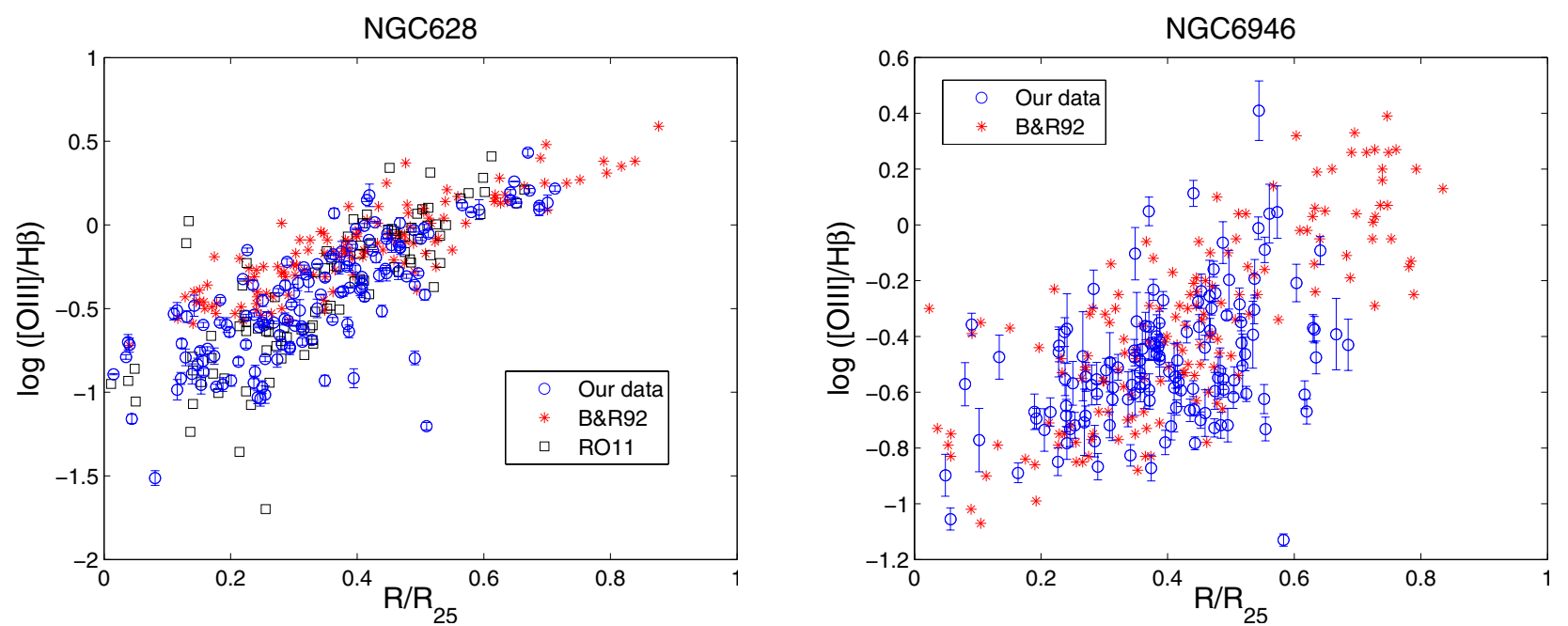

Fig. 8. Logarithm of the $[\mathrm{OIII}] / \mathrm{H} \beta$ as a function of the $R_{25}$ radius for NGC 628 (left panel) and NGC 6946 (right panel). Our data are open circles, asterisks are the data from $\mathrm{B} \& \mathrm{R} 92$, and open squares are the data from RO11. 

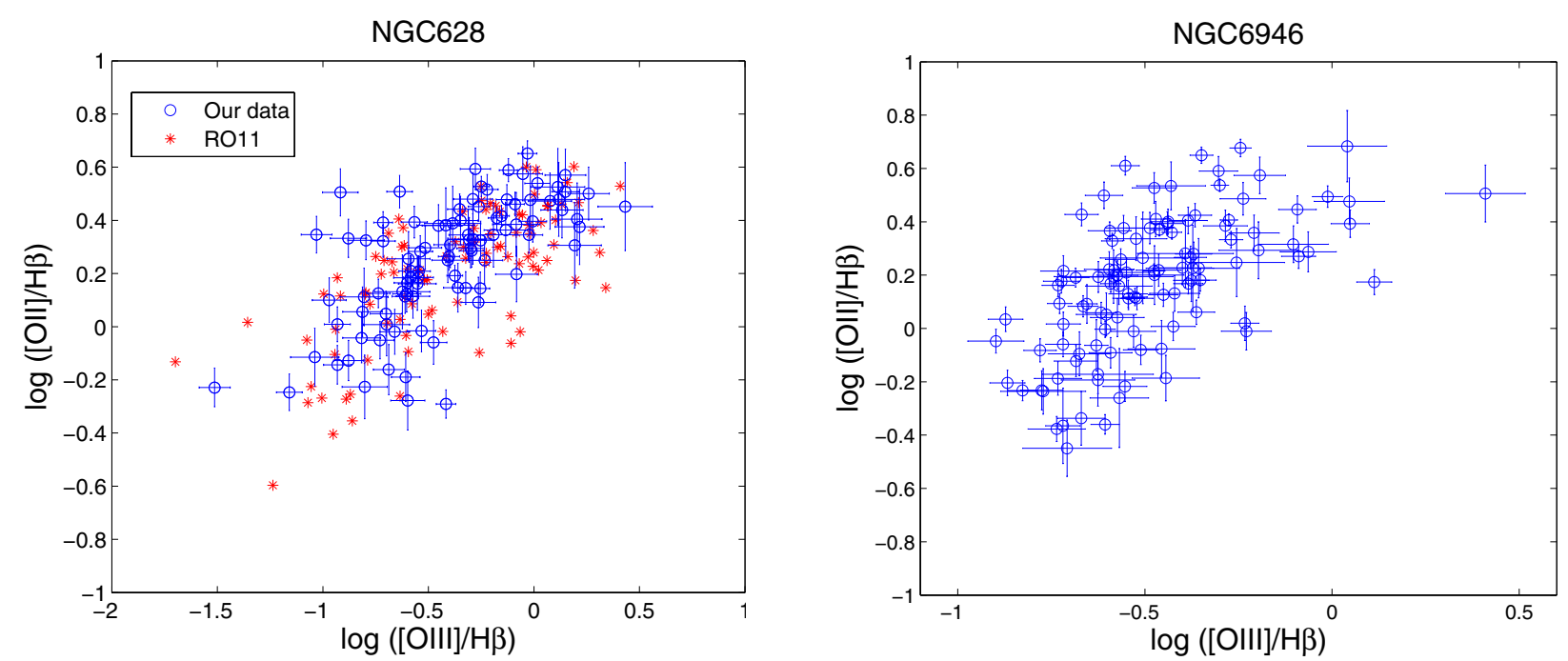

Fig. 9. Logarithm of the $[\mathrm{OII}] / \mathrm{H} \beta$ versus of the logarithm of $[\mathrm{OIII}] / \mathrm{H} \beta$ for NGC 628 (left panel) and NGC 6946 (right panel). Our data are open circles, asterisks are the data from RO11.

seeems to be somewhat lower (at least in the parts of the galaxy where we have data) when compared with NGC 628.

\section{Determination of the oxygen abundance}

\subsection{The problem with empirical calibrations}

The main method employed to derive the metallicity of galactic $\mathrm{H}$ II regions is based on electron-temperature sensitive lines, the so-called direct method (e.g. Searle 1971; Garnett \& Kennicutt 1994; Kennicutt et al. 2003; Izotov et al. 2006). However, the oxygen line [OIII] $\lambda 4363$, which is critical for determining the electron temperature (Osterbrock \& Ferland 2006; or Izotov et al. 2006), is weak and difficult to detect in the inner parts of spiral galaxies, where the oxygen abundance is high. Hence to determine the metallicity with nebular lines, we need alternative methods based on empirical calibrations. Several calibrations have been proposed in the literature: Edmunds \& Pagel (1984), McCall et al. (1985), Zaritsky et al. (1994), Kewley \& Dopita (2002), and Kobulnicky \& Kewley (2004). These are based on the $R_{23}$ parameter, defined as

$R_{23}=\frac{[\mathrm{OII}] \lambda \lambda 3727,29+[\mathrm{OIII}] \lambda \lambda 4959,5007}{\mathrm{H} \beta}$.

Pilyugin (2001a,b) and Pilyugin \& Thuan (2005) also propose that this involves the $R_{23}$ parameter and the excitation $P$, defined as

$$
P=\frac{[\mathrm{OIII}] \lambda \lambda 4959,5007}{[\mathrm{OII}] \lambda \lambda 3727,29+[\mathrm{OIII}] \lambda \lambda 4959,5007} .
$$

Van Zee et al. (1998), Denicoló et al. (2002) and Pettini \& Pagel (2004) proposed the use of a calibration with the parameter $N_{2}$.

The data set presented here allows us to obtain only the $R_{23}$ and $P$ parameters. However, it has to be pointed out that the $R_{23}$ calibrations provide oxygen abundance determinations that are about 0.5 dex higher than those obtained by the direct method (López-Sánchez \& Esteban 2010; Cedrés et al. 2004). According to López-Sánchez \& Esteban (2010), the Pilyugin \& Thuan (2005, hereafter PT05) calibration is the most suitable for deriving oxygen abundances because it is in closer agreement with the metallicity obtained by the direct method. On the other hand, Moustakas et al. (2010) argue that the scarcity of calibration

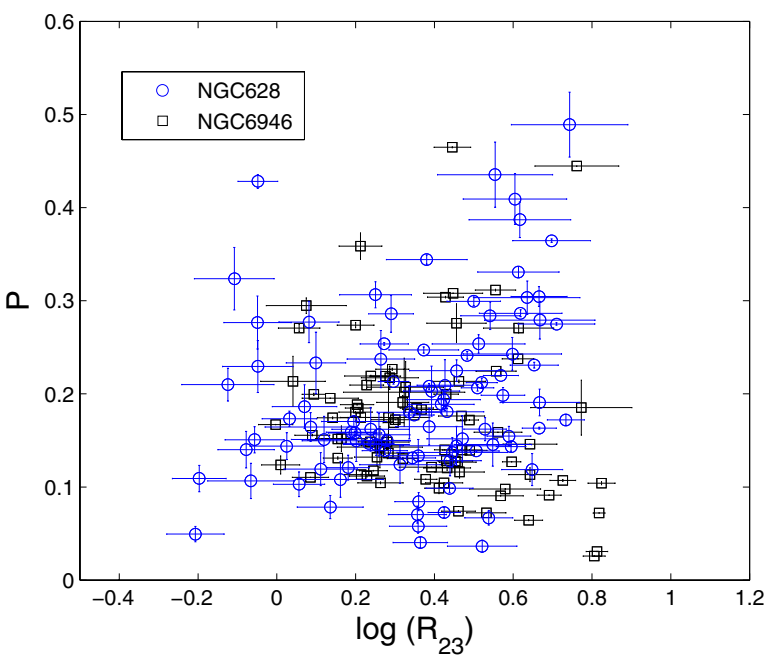

Fig. 10. Excitation $P$ (Eq. (2)) as a function of the logarithm of $R_{23}$ for NGC 628 (open circles) and NGC 6946 (open squares).

points with high oxygen abundance and low excitation used in the development of the PT05 method may cause some problems in the metallicity obtained, an effect that was also recognized in PT05. Moreover, Moustakas et al. (2010) also recommend employing two calibrations for SINGS galaxies, Kobulnicky \& Kewley (2004, hereafter KK04) and PT05, or their average, in order to diminish the possible systematic errors introduced by each one.

In Fig. 10, we have presented for NGC 628 and NGC 6946 the $P$ parameter as a function of the logarithm of $R_{23}$. For almost all our regions, $P<0.4$, so that they are located within a range of $P$ that it is not well-sampled, because for PT05 the greater number of calibration points are in $P>0.6$ for the upper branch of the metallicity. However, in Cedrés et al. (2004), it is shown that even for M 101 regions with a low value of $P$, the calibration of Pilyugin (2001a,b), which is a previous version of the present PT05, ensures a more than reasonable agreement with the metallicity obtained by the direct method. This seems to indicate that for low values of $P$, abundances inferred using PT05 may have more scatter, but that the mean oxygen abundance value determined will behave as well as that for higher values of $P$, where more calibration points are present. 
Table 8. Values for the parameters $P$ and $R_{23}$, and the metallicity derived through PT05 method for the first ten H II regions of NGC 628.

\begin{tabular}{cccc}
\hline \hline Number & $P$ & $\log R_{23}$ & $12+\log (\mathrm{O} / \mathrm{H})$ \\
\hline 1 & 0.36 & 0.70 & $8.27 \pm 0.02$ \\
2 & 0.29 & 0.62 & $8.28 \pm 0.04$ \\
3 & 0.30 & 0.64 & $8.28 \pm 0.16$ \\
4 & - & - & - \\
5 & - & - & - \\
6 & - & - & - \\
7 & 0.41 & 0.60 & $7.98 \pm 0.22$ \\
8 & - & - & - \\
9 & 0.44 & 0.55 & $7.98 \pm 0.27$ \\
10 & - & - & - \\
\hline
\end{tabular}

Notes. The complete table is available electronically.

The metallicity is derived, following PT05 method, for the upper branch using

$$
12+\log (\mathrm{O} / \mathrm{H})_{\text {up }}=\frac{R_{23}+726.1+842.2 P+337.5 P^{2}}{85.96+82.76 P+43.98 P^{2}+1.793 R_{23}}
$$

and for the lower branch using

$$
12+\log (\mathrm{O} / \mathrm{H})_{\mathrm{low}}=\frac{R_{23}+106.4+106.8 P-3.40 P^{2}}{17.72+6.60 P+6.95 P^{2}-0.302 R_{23}} .
$$

To obtain the metallicity using the KK04 calibration, we apply the equations

$$
\begin{gathered}
12+\log (\mathrm{O} / \mathrm{H})_{\mathrm{up}}=9.72-0.777 x-0.951 x^{2}-0.072 x^{3}-0.811 x^{4} \\
-\log (q)\left(0.0737-0.0713 x-0.141 x^{2}+0.0373 x^{3}-0.058 x^{4}\right)
\end{gathered}
$$

$$
\begin{aligned}
12+\log (\mathrm{O} / \mathrm{H})_{\text {low }}= & 9.40+4.65 x-3.17 x^{2} \\
& -\log (q)\left(0.272+0.547 x-0.513 x^{2}\right)
\end{aligned}
$$

where $x=\log \left(R_{23}\right)$ and $q$ is given by

$$
\begin{aligned}
\log (q)= & 32.81-1.153 y^{2}+z(-3.396-0.025 y \\
& \left.+0.1444 y^{2}\right) \times\left[4.603-0.3119 y-0.163 y^{2}\right. \\
& \left.+z\left(-0.48+0.0271 y+0.02037 y^{2}\right)\right]^{-1}
\end{aligned}
$$

where $z=12+\log (\mathrm{O} / \mathrm{H})$ and

$y=\log \left(\frac{[\mathrm{OIII}] \lambda \lambda 4959,5007}{[\mathrm{OII}] \lambda 3727}\right)$.

In this study, we obtain the metallicity employing the PT05 method and compare it with the results from KK04 and, where available, metallicity values obtained through the direct method. In Tables 8 and 9 , the values for the parameters $P$ and $R_{23}$, and the oxygen abundance derived using the PT05 calibration for the first 10 H II regions of NGC 628 and NGC 6946, respectively, are presented.

\subsection{The metallicity of NGC 628}

From Fig. 10, using the PT05 calibration, we can observe that most regions detected for NGC 628 are in the $0.1<P<0.3$ regime. In PT05, the authors situated the transition zone between the upper and the lower branch of the calibration, approximately
Table 9. Values for the parameters $P$ and $R_{23}$, and the metallicity derived through PT05 method for the first ten H II regions of NGC 6946.

\begin{tabular}{lccc}
\hline \hline Number & $P$ & $\log R_{23}$ & $12+\log (\mathrm{O} / \mathrm{H})$ \\
\hline 1 & - & - & - \\
2 & 0.10 & 0.58 & $8.05 \pm 0.13$ \\
3 & 0.22 & 0.56 & $8.27 \pm 0.06$ \\
4 & - & - & - \\
5 & 0.09 & 0.57 & $8.05 \pm 0.08$ \\
6 & 0.24 & 0.61 & $8.22 \pm 0.05$ \\
7 & 0.10 & 0.42 & $8.22 \pm 0.05$ \\
8 & 0.12 & 0.39 & $8.27 \pm 0.08$ \\
9 & 0.07 & 0.53 & $8.06 \pm 0.06$ \\
10 & - & - & - \\
\hline
\end{tabular}

Notes. The complete table is available electronically.

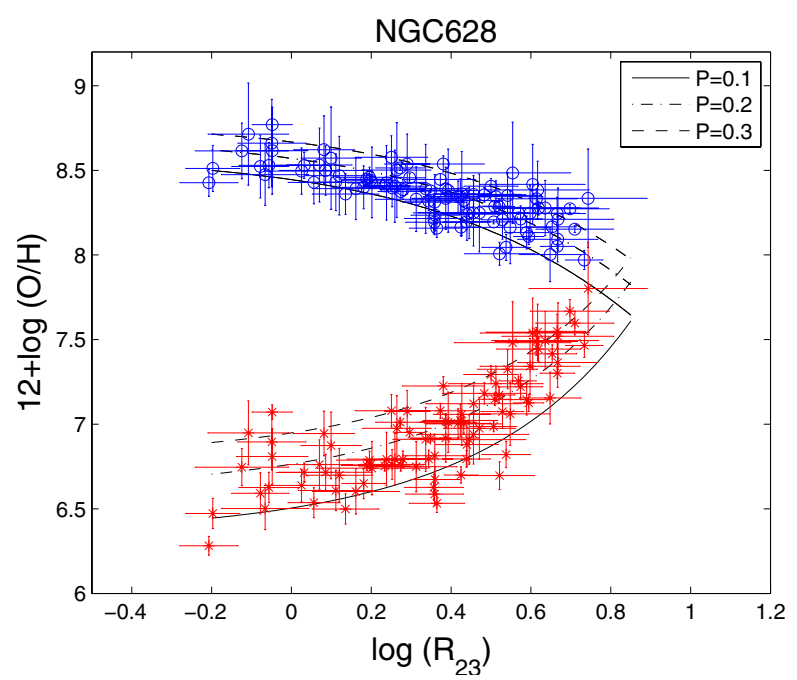

Fig. 11. Oxygen abundance for NGC 628 as a function of the logarithm of $R_{23}$ for different values of $P$. Open circles are the regions with the metallicity derived using the upper branch calibration. Asterisks are the regions with metallicities derived using the lower branch calibration.

in the range $8.0<12+\log (\mathrm{O} / \mathrm{H})<8.3$. However, this is generally a zone populated by regions with a wide range of values of $P$, and with most of the regions with $P<0.5$. Taking into account that our values of $P$ are more restricted, that the transition zone for low values of $P$ is below $12+\log (\mathrm{O} / \mathrm{H})=8.0$, and that we sample only the central parts of the galaxy, where the oxygen abundance is moderately high, we can assume that all the regions with $8.0<12+\log (\mathrm{O} / \mathrm{H})<8.3$ belong to the upper branch of the metallicity calibration (Fig. 11).

In Fig. 12, we show the oxygen abundance obtained using PT05 calibration for the H II regions of NGC 628 versus galactocentric radius. There is, again, reasonable agreement between our data and RO11 data. We have also presented data from Castellanos et al. (2002) (filled triangle, region H13), where the oxygen abundance was obtained employing the direct method, or regions where an empirical $S_{23}$ calibration was used (open triangles). If we fit our data using the least squares method, we obtain a gradient at the galactocentric radius of $-0.36 \mathrm{dex} / R_{25}$, which is very similar to that obtained with the RO11 data, of $-0.38 \mathrm{dex} / R_{25}$.

In Fig. 13, we have the metallicity for NGC 628 as in Fig. 12 but, for comparison, we have included the metallicity obtained using the KK04 calibration for our data (open circles) and those of RO11 (open squares). 


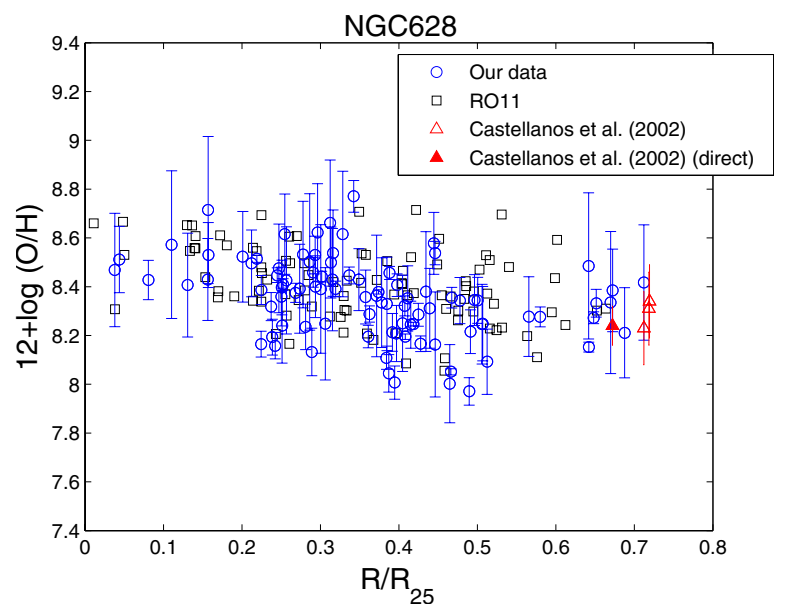

Fig. 12. Oxygen abundance for NGC 628 as a function of the galactocentric radius, employing the calibration from PT05. Our data are the open circles. The open squares are the data from RO11 and the open and filled triangles are the data from Castellanos et al. (2002).

It is clear that our data closely follow the same behaviour as RO11 data. There is also a noticeable difference between the oxygen abundances yielded for both calibrations, which can be as large as 0.6 dex in the inner parts of the galaxy. As expected, the KK04 calibration defines a tighter correlation with galactocentric radius, while PT05 infers value with a wider dispersion. This is because most of the $\mathrm{H}$ II regions in the inner parts of NGC 628 have $P<0.4$ and, as mentioned previously, this range is not well-sampled by the PT05 calibration. However, if we take into account the position of the regions from Castellanos et al. (2002) in the figure, it is clear that they lie closer to the gradient drawn by the PT05 calibration than that of KK04. This agrees with the results of Cedrés et al. (2004) and López-Sánchez \& Esteban (2010).

In Table 10, we summarise the gradients (expressed as $\operatorname{dex} / R_{25}$ ) and the central oxygen abundances obtained for NGC 628 using both calibrations and three different datasets: Moustakas et al. (2010), RO11, and our data. There is overall agreement between the three datasets within the uncertainties for both calibrations. We must stress that Moustakas et al. (2010) data were obtained through long-slit spectroscopy, and that the RO11 data were obtained using integral-field spectroscopy methods, while our data were obtained using narrow-band imaging. This implies that narrow-band imaging techniques are at least as accurate as, and less time-consuming than, any spectroscopic method (long-slit or integral-field).

To explore the azimuthal behaviour of the oxygen abundance, we created a 2D continuous model of the metallicity from our discrete $\mathrm{H}$ II regions, using a Delaunay triangulation (Barber et al. 1996). To ensure a sufficent number of datapoints to make the required interpolations, we have included in the grid all the regions detected, even those with fluxes below the $3 \sigma$ criterion for [OII] and [OIII]. In Fig. 14, we present the metallicity contours of NGC 628, using the PT05 calibration (left panel) and KK04 calibration (right panel). The results from the PT05 calibration are similar to those obtained by Sánchez et al. (2011). There are several knots with higher metallicity values that seem to be associated with the arms. However, those knots disappear for the KK04 calibration, where a smoother and clearer gradient is shown instead.

To highlight the high metallicity knots and possible azimuthal variations, we have subtracted the radial metallicity gradient from the disc. The residuals are shown in Fig. 15.

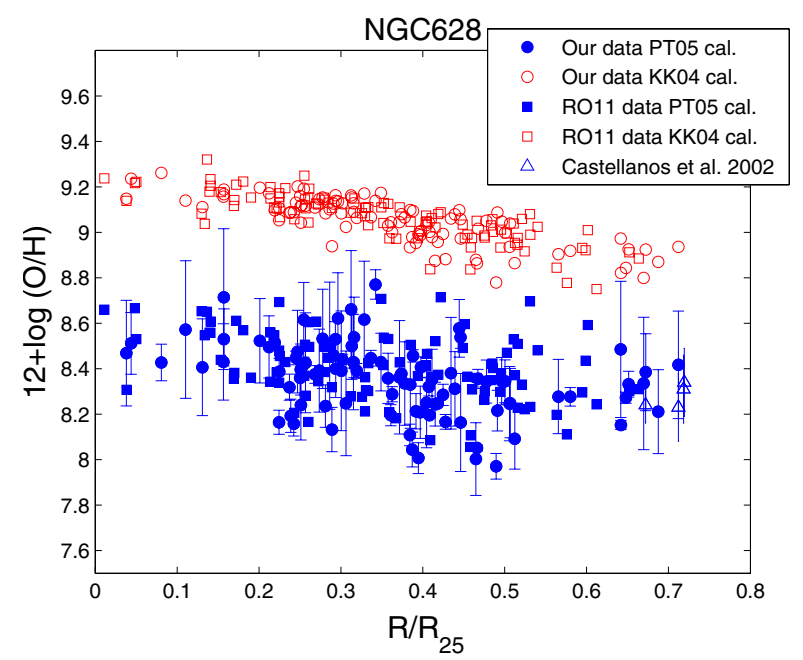

Fig. 13. Oxygen abundance for NGC 628 as a function of galactocentric radius, using the calibration from PT07 (filled circles and squares) and KK04 calibration (open circles and squares). Data from Castellanos et al. (2002) are the open triangles.

Taking into account that the main difference between both calibrations (PT05 and KK04) is the inclusion of the $P$ parameter in the PT05 equations, to help us interpret those high metallicity knots, we created another model in two dimensions for the galaxy, but for the parameter $P$, again using a Delaunay triangulation. From Fig. 16, some of the knots seem to be "caused" by a higher value of $P$. We have to take into account that for regions with the same value of $R_{23}$, a higher value of $P$ means a higher metallicity.

These knots seem to be associated with the arms, since their mean azimuthal distance is $\sim 0.4 \mathrm{kpc}$. The parameter $P$ is directly related to the temperature of the ionizing cluster of the $\mathrm{H}$ II region (see Pilyugin 2001a). For higher values of $P$, the temperature increases. Hence it is possible for the knots in Fig. 16 to be due to the presence of more high temperature stars in the clusters that ionize the $\mathrm{H}$ II region; in other words, the initial mass function (IMF) for those knots contains a greater fraction of more massive stars than the rest of the H II regions of the disc. Variations in the IMFs among galaxies, linked to the strength of the density wave, have previously been reported (Cedrés et al. 2005); these kinds of variations are not entirely unexpected.

\subsection{The metallicity of NGC 6946}

Unfortunately, for NGC 6946 there is no other study with the same emission lines as the present one and the excellent work of RO11 for NGC 628. Moreover, we could not find in the literature any data with information on the auroral oxygen lines to derive the metallicity with the direct method, so we are restricted to making comparisons only with the results of Moustakas et al. (2010). From Fig. 10, it is clear that the H II regions of NGC 6946 are similar in behaviour to NGC 628 ones, with low values of $P$, so in the determination of their oxygen abundances we need only use the upper branch of the PT05 calibration (Fig. 17).

In Fig. 18, we have presented the metallicity for NGC 6946, based on PT05 calibration (filled circles) and KK04 calibration (open circles). The behaviour of the metallicity is the same as for NGC 628, with the KK04 calibration yielding higher values of metallicity than those obtained using the PT05 calibration. The difference between both is about $0.5-0.6$ dex at maximum. 
Table 10. Radial oxygen abundance gradients and central oxygen abundances for NGC 628.

\begin{tabular}{l|cccc}
\hline \hline & \multicolumn{2}{|c}{ Gradient $\left(\mathrm{dex} / R_{25}\right)$} & \multicolumn{2}{c}{ Central metallicity } \\
& PT05 & KK04 & PT05 & KK04 \\
\hline This work & $-0.36 \pm 0.1$ & $-0.57 \pm 0.1$ & $8.51 \pm 0.04$ & $9.25 \pm 0.04$ \\
Moustakas et al. (2010) & $-0.27 \pm 0.05$ & $-0.57 \pm 0.04$ & $8.43 \pm 0.02$ & $9.19 \pm 0.02$ \\
RO11 & $-0.38 \pm 0.02$ & $-0.68 \pm 0.03$ & $8.54 \pm 0.01$ & $9.31 \pm 0.02$ \\
\hline
\end{tabular}
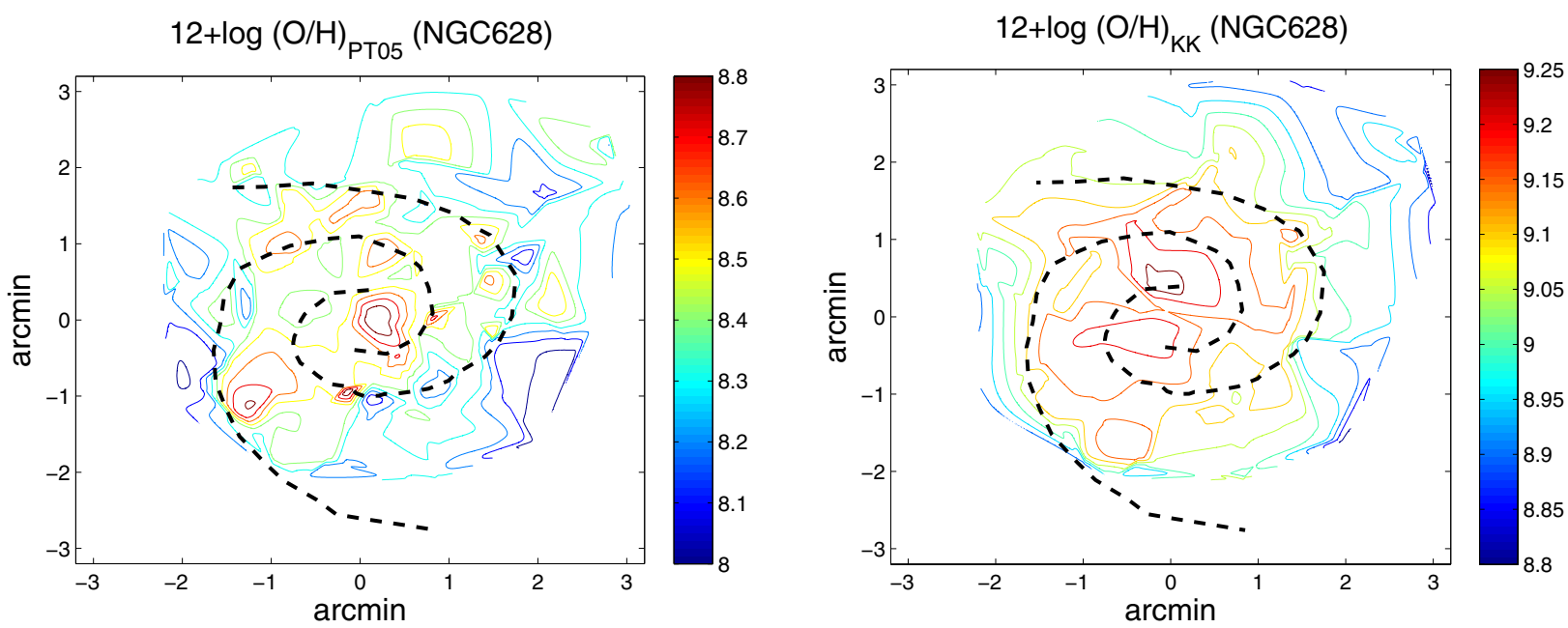

Fig. 14. Contour plot of the oxygen abundance determined using the PT05 calibration (left panel) and the KK04 calibration (right panel) for NGC 628. North is at the top and east is to the left. Spiral arms are indicated by the dashed lines (Cedrés et al., in prep.).

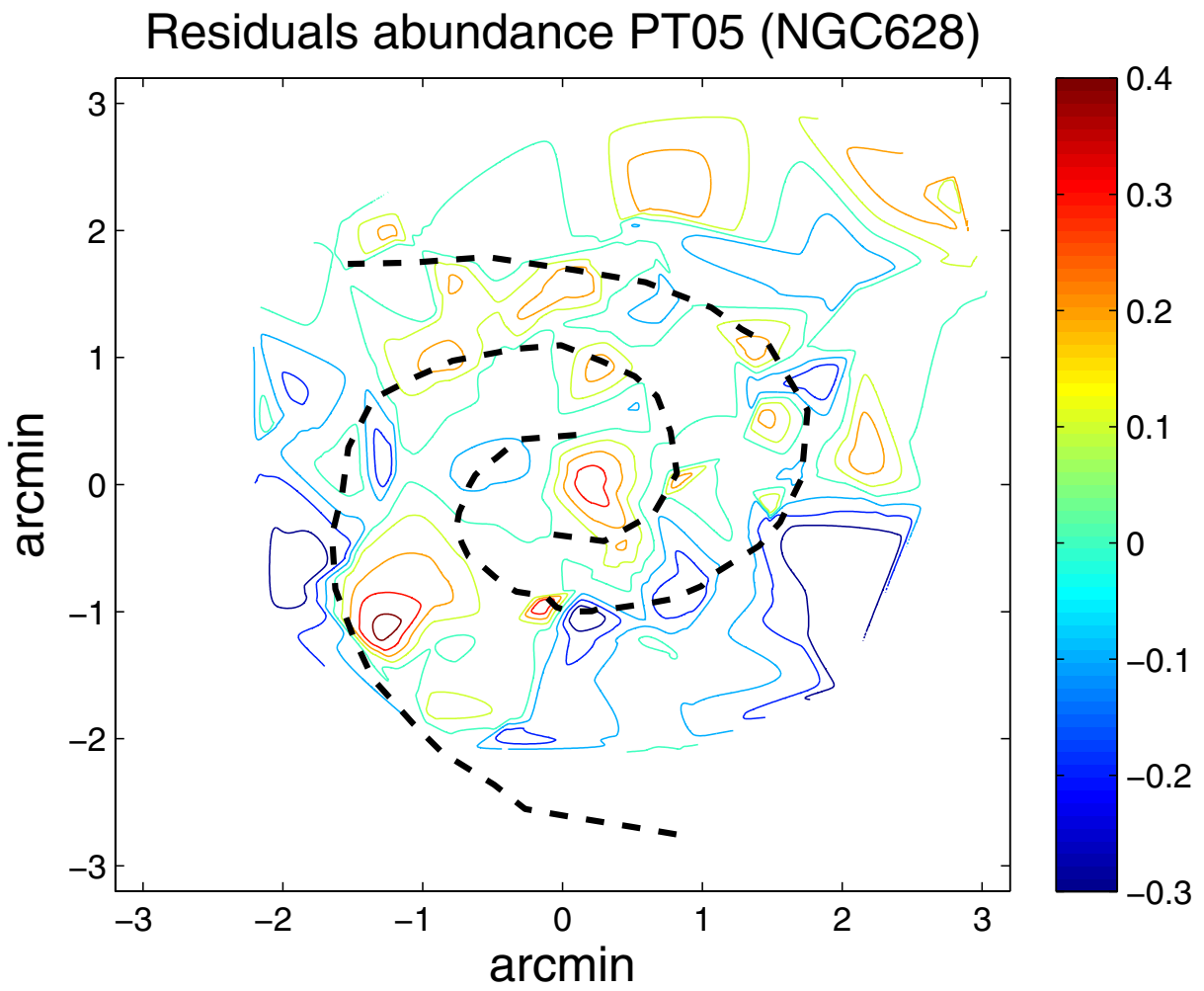

Fig. 15. Contour plot of the residuals in the oxygen abundance, determined using the PT05 calibration, after subtraction of the mean radial metallicity gradient, for NGC 628. North is at the top and east to the left. Spiral arms are indicated by the dashed lines (Cedrés et al., in prep.).

In Table 11 a summary of the gradients and central oxygen abundances obtained for NGC 6946 and the results from Moustakas et al. (2010) is shown. There is a large discrepancy between our results and those of Moustakas et al. (2010) in the gradient derived using PT05 calibration. This may be due to the small number of $\mathrm{H}$ II regions (only six for this galaxy) used by Moustakas et al. (2010) to derive the gradient. However, taking into account the uncertainties, both results are still compatible. Such a discrepancy does not exist for the KK04 calibration, probably owing to the smaller dispersion in the data. 


\section{$P(N G C 628)$}

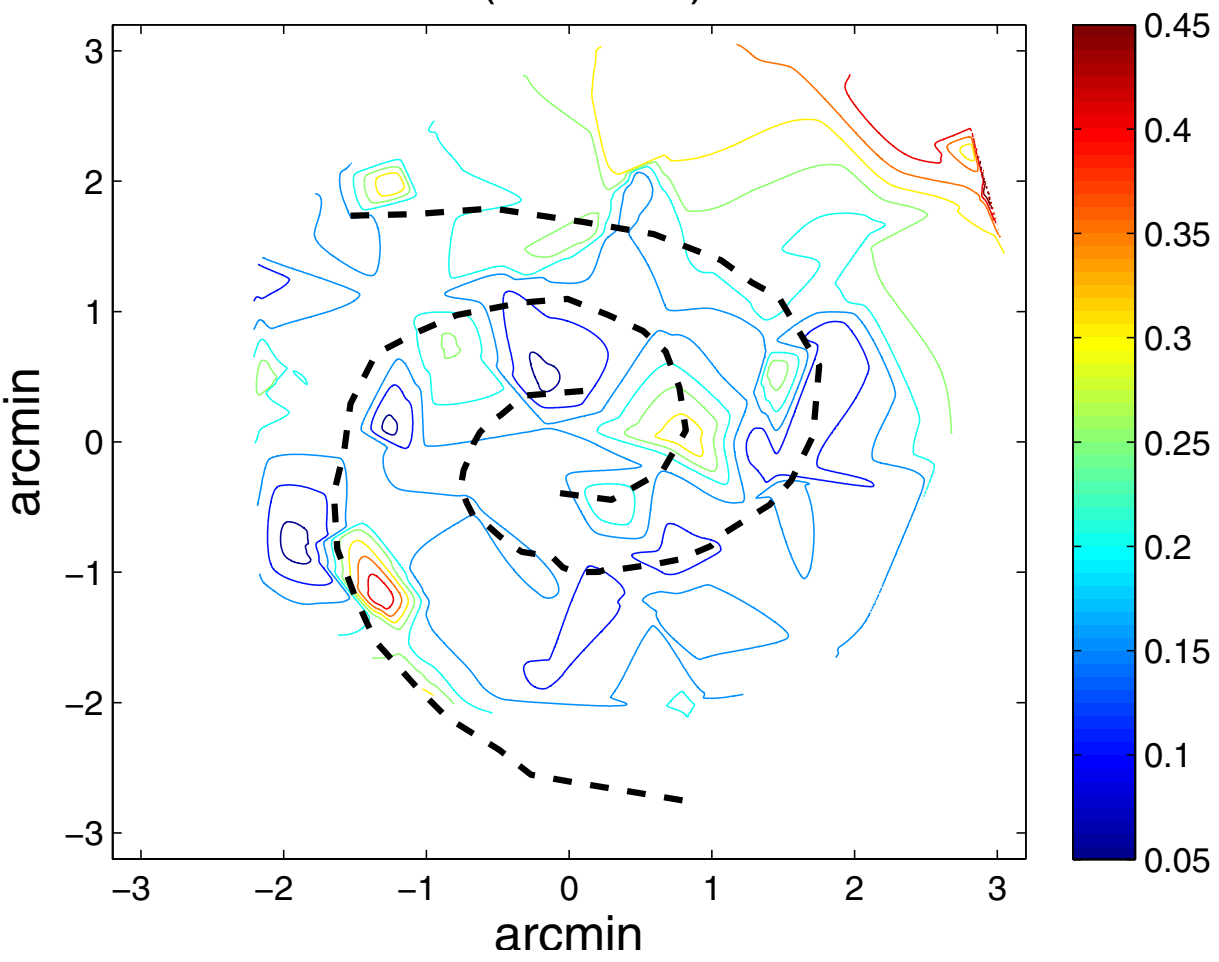

Fig. 16. Contour plot of the parameter $P$ for NGC 628. North is at the top and east is to the left. Spiral arms are indicated by the dashed lines (Cedrés et al., in prep.).

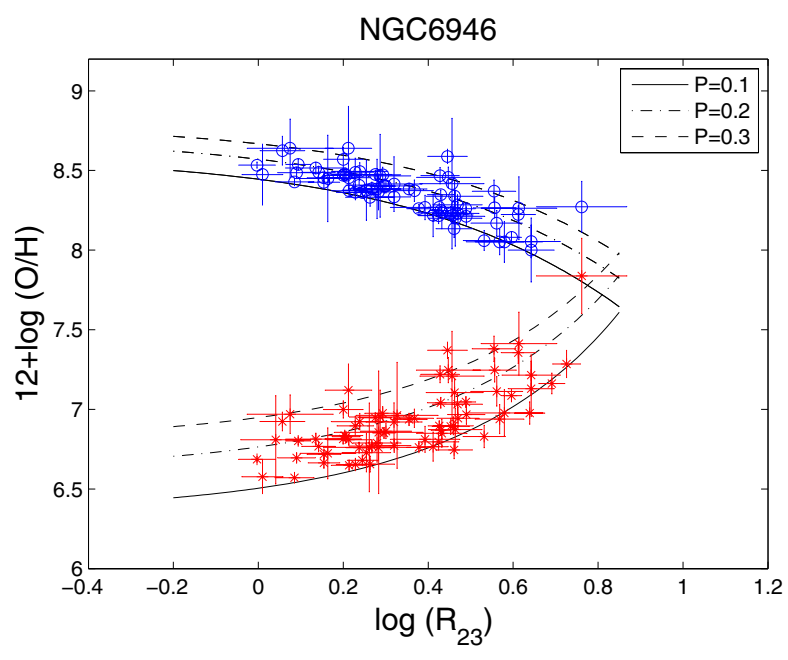

Fig. 17. Oxygen abundance for NGC 6946 as a function of the logarithm of $R_{23}$ for different values of $P$. Open circles are the regions with the metallicity derived using the upper branch calibration. Asterisks are the regions with metallicity derived using the lower branch calibration.

We created a 2D model for the oxygen abundance of NGC 6946, with the same conditions as for NCG 628.

As seen in Fig. 19, there is a clear azimuthal structure in the behaviour of the metallicity for NGC 6946. This structure is clearer in the data from the PT05 calibration, where a highmetallicity spiral arm seems to exist. This higher metallicity arm seems to be associated with several stellar arms, first to the north and north-south arms, and then following the south and southnorth arms. This behaviour also exists in the KK04 calibration data, although it is less clear.

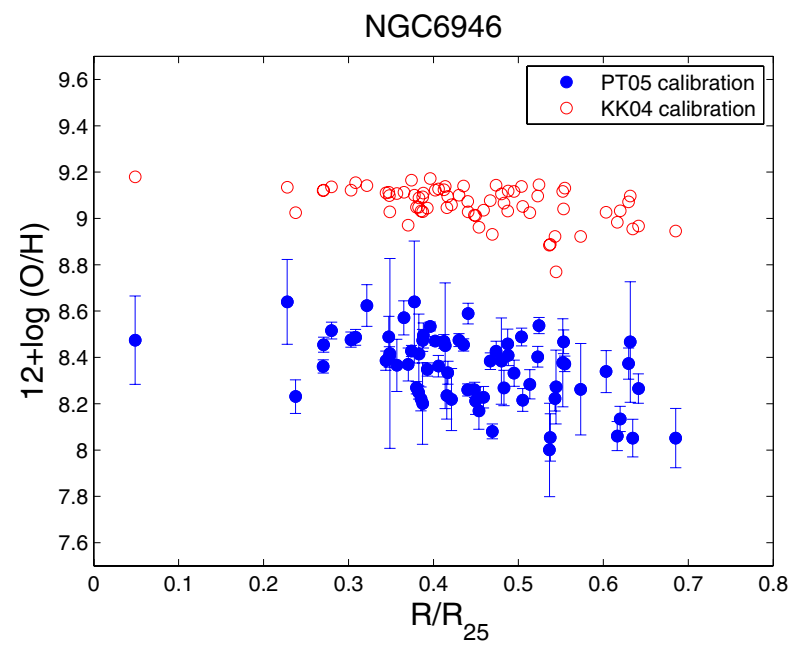

Fig. 18. Oxygen abundance for NGC 6946 as a function of galactocentric radius, using the calibrations from PT07 (filled circles) and KK04 (open circles).

In Fig. 20, we obtain a clearer view of the azimuthal variations for NGC 6946, after subtracting the metallicity radial gradient.

To explore this behaviour, we again created a model for the parameter $P$, using the same conditions as for the oxygen abundance. The results are shown in Fig. 21. There are several knots with high $P$ that follow the same path as the higher metallicity arm, so, as in the case of NGC 628 with the knots, the higher metallicity arm seems to be generated by the behaviour of $P$, which generates an arm that is associated with the main stellar arm (Foyle at al. 2010), but does not exactly follow the same path. As suggested by Cedrés et al. (in prep.), there is a 
Table 11. Radial metallicity gradients and central oxygen abundances for NGC 6946.

\begin{tabular}{l|cccc}
\hline \hline & \multicolumn{2}{|c}{ Gradient $\left(\mathrm{dex} / R_{25}\right)$} & \multicolumn{2}{c}{ Central metallicity } \\
& PT05 & KK04 & PT05 & KK04 \\
\hline This work & $-0.40 \pm 0.1$ & $-0.29 \pm 0.1$ & $8.57 \pm 0.04$ & $9.19 \pm 0.04$ \\
Moustakas et al. (2010) & $-0.17 \pm 0.15$ & $-0.28 \pm 0.1$ & $8.45 \pm 0.06$ & $9.13 \pm 0.04$ \\
\hline
\end{tabular}
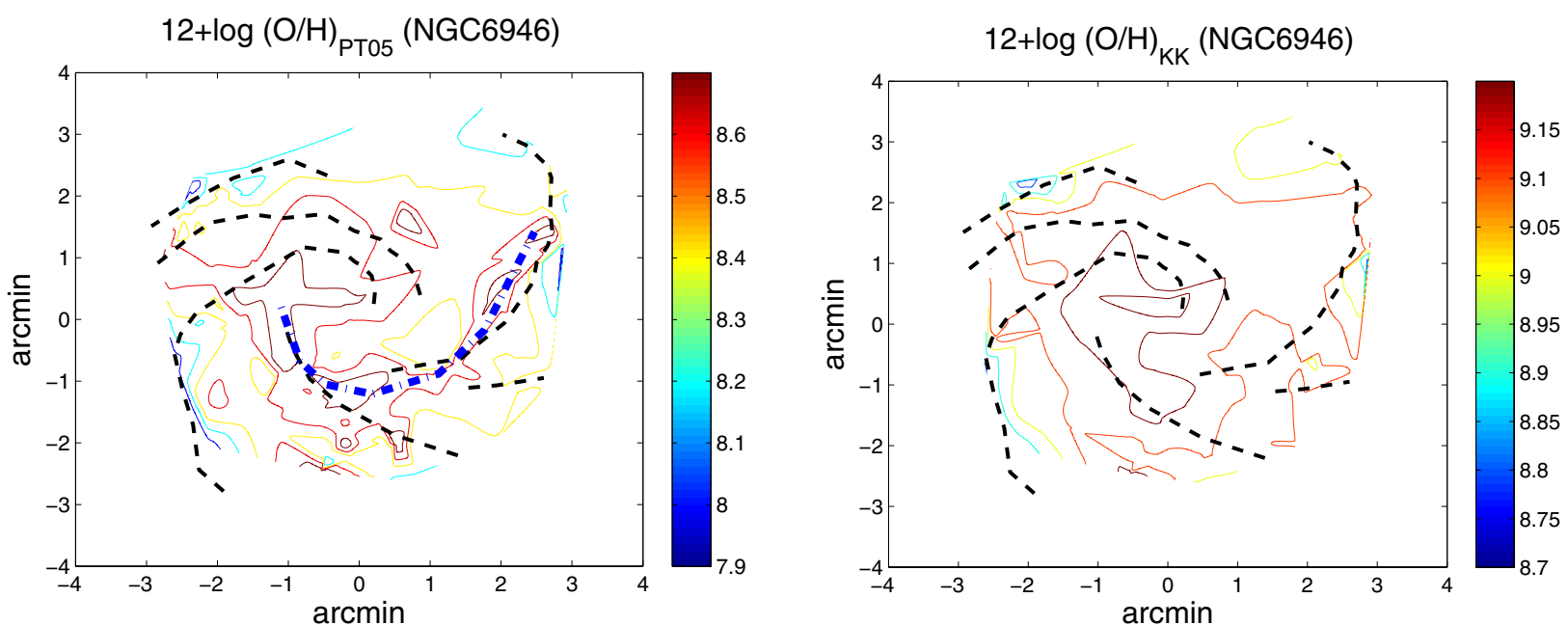

Fig. 19. Contour plot of the metallicity, determined using the PT05 calibration (left panel) and the KK04 calibration (right panel), for NGC 6946. North is at the top, east is to the left. Spiral arms are indicated by the dashed lines (Cedrés et al., in prep.). The high-metallicity arm is indicated in the PT05 calibration figure by the point-dashed line.

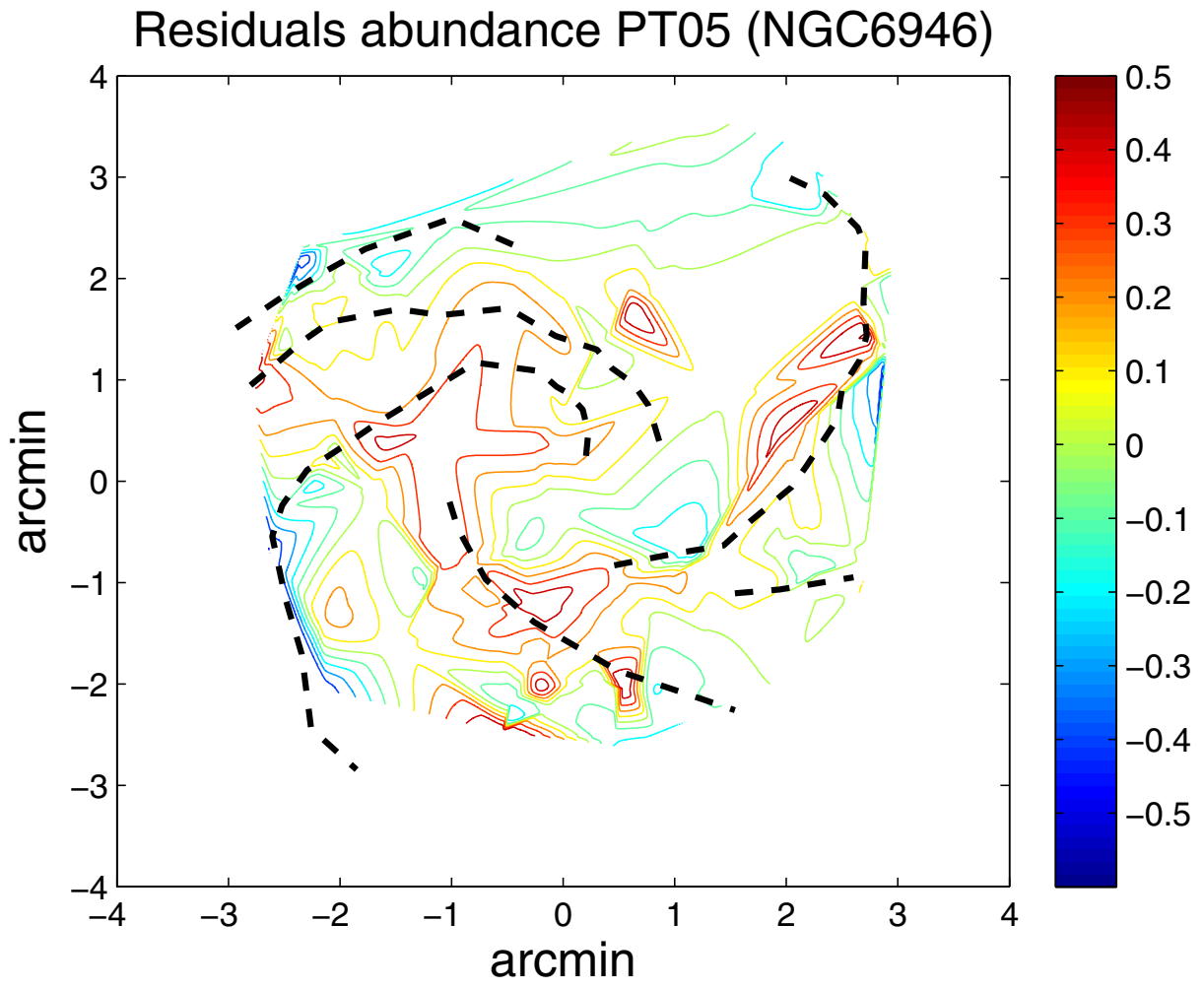

Fig. 20. Contour plot of the residuals of the oxygen abundance, determined using the PT05 calibration, after the subtraction of the radial metallicity gradient, for NGC 6946. North is at the top and east is to the left. Spiral arms are indicated by the dashed lines (Cedrés et al., in prep.).

difference in the position between the stellar arm and the $\mathrm{H}$ II regions, as it is more likely to find star-forming regions "behind" the stellar arms (if we consider the direction of rotation of the galaxy) instead of being at the centre of them.
The same case as for NGC 628 can be made for this galaxy in order to explain the "arm" in $P$ and oxygen abundance: it could be due to an accumulation of regions with a slightly different IMF from those in the rest of the disc of the galaxy. 


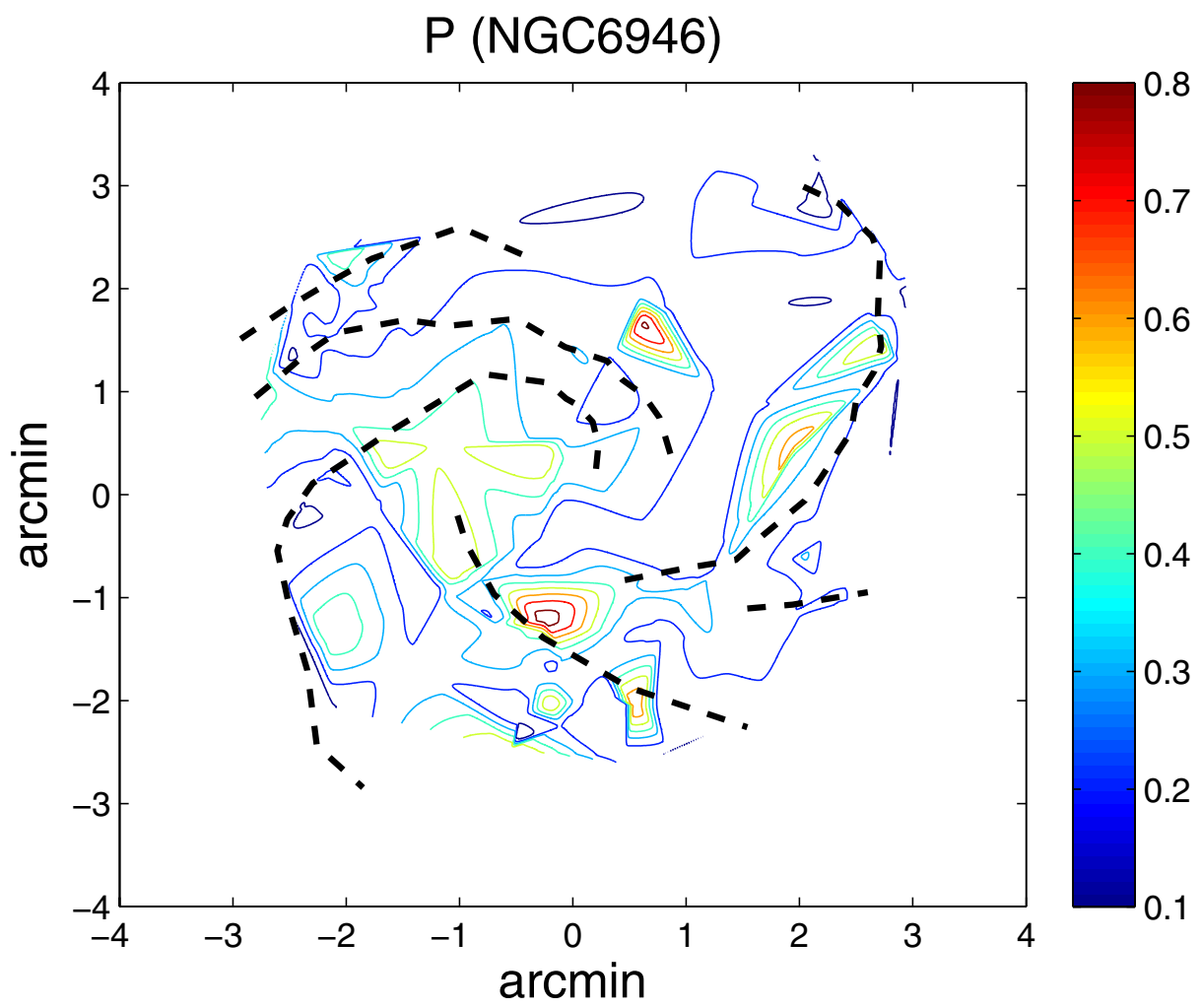

Fig. 21. Contour plot of the parameter $P$ for NGC 6946. North is at the top, east is to the left. Spiral arms are indicated by the dashed lines (Cedrés et al., in prep.).

\section{Conclusions}

We have obtained data from 209 and $226 \mathrm{H}$ II regions from the galaxies NGC 628 and NGC 6946, respectively, in the emission lines $\mathrm{H} \alpha, \mathrm{H} \beta,[\mathrm{O}$ II] $] 3727$, and [O III] $\lambda \lambda 4959,5007$ and their respective continua. For each of these regions, the isophotal area, extinction, EW, and oxygen abundance were derived.

We have found that the $\mathrm{H} \alpha \mathrm{EW}$ for NGC 6946 is slightly broader than that derived for NGC 628 . This result could be due to the presence of, on average, older H II regions in NGC 628 than NGC 6946. In general, the radial behaviour of the EW for both galaxies agrees with the trend observed in other galaxies (Cedrés \& Cepa 2002).

The $[\mathrm{O} \mathrm{II}] / \mathrm{H} \beta$ and $[\mathrm{O} \mathrm{III}] / \mathrm{H} \beta$ line ratios were obtained for regions where the $[\mathrm{O}$ II $]$ and $[\mathrm{O}$ III] emission were detectable ( $3 \sigma$ above the background noise). Our data was then compared with the results presented in the literature and general agreement was found, although we were able to obtain data for a greater number of $\mathrm{H}$ II regions with a lower investment in observing time, compared with other studies.

Radial oxygen-abundance gradients for both galaxies, using the PT05 and KK04 calibration, were obtained. These gradients were compared with others derived from the literature and again a general agreement was found.

As expected, the KK04 method yields a higher metallicity than and a different gradient from the PT05 method. Even if the PT05 method produces a larger dispersion than that for the KK04 method, the gradient and the metallicity values for single regions are closer to the direct-method results, as previously suggested in the literature (e.g. Cedrés et al. 2004; or López-Sánchez \& Esteban 2010).

Two-dimensional contour models for the metallicity (and associated parameters) for both galaxies were created using the Delaunay triangulation. For both galaxies, azimuthal structures in the metallicity were discovered: for NGC 628, there were high oxygen-abundance knots linked to the arms; for NGC 6946, a high metallicity "arm" was found. The knots for NGC 628 are approximately in the same position as those presented by Sánchez et al. (2011). These asymmetries are not as significant when the KK04 calibration is used and appear to be linked to the behaviour of the parameter $P$.

Taking into account the relationship that exists between $P$ and the effective temperature of the ionizing clusters, it is possible that these high oxygen-abundance/high $P$ regions may correspond to a higher value of the effective temperature and therefore, a larger fraction of massive stars than regions with lower $P$, which also indicates that there is a variation in the IMF across these regions. When we take into account that the high metallicity knots (and the high metallicity arm) are associated with the galaxies' stellar arms, we are able to propose a relationship between them and the density wave. This result supports the conclusions of Cedrés et al. (2004), who suggested that the density wave may have an influence on the IMF. However, to confirm this result, more observations of galaxies with different kinds of arms will be necessary.

Acknowledgements. This work was supported by the Spanish Ministry of Economy and Competitiveness (MINECO) under the grant AYA2011-29517C03-01. Based on observations made with the Nordic Optical Telescope, operated on the island of La Palma jointly by Denmark, Finland, Iceland, Norway, and Sweden, in the Spanish Observatorio del Roque de los Muchachos of the Instituto de Astrofísica de Canarias. B.C. wishes to thank Dr. César Esteban and Dr. L.S. Pilyugin for their useful advice and suggestions during the completion of this work.

\section{References}

Barber, C. B., Dobkin, D. P., \& Huhdanpaa, H. T. 1996, ACM Trans. Math. Soft., 22, 469

Barth, C. S., Cepa, J., Vílchez, J. M., \& Dottori, H. A. 1994, AJ, 108, 2069 Belley, J., \& Roy, J-N. 1992, ApJS, 78, 61 (B\&R92) 
Bresolin, F., Kennicutt, R. C., \& Garnett, D. R. 1999, ApJ, 510, 104 Castellanos, M., Díaz, Á. I., \& Terlevich, E. 2002, MNRAS, 329, 315 Cedrés, B., \& Cepa, J. 2002, A\&A, 391, 809

Cedrés, B., Urbaneja, M. A., \& Cepa, J. 2004, A\&A, 422, 511

Cedrés, B., Cepa, J., \& Tomita, A. 2005, ApJ, 634, 1043

Denicoló, G., Terlevich, R., \& Terlevich, E. 2002, MNRAS, 330, 69

de Vaucouleurs, G., de Vaucouleurs, A., Corwin Jr., H. G., et al. 1991, Third

Reference Catalogue of Bright Galaxies, Version 3.9 (Berlin: Springer)

Edmunds, M. G., \& Pagel, B. E. J. 1984, MNRAS, 211, 507

Elmegreen, D. M., \& Elmegreen, B. G. 1987, ApJ, 314, 3

Epinat, B., Amram, P., Marcelin, M., et al. 2008, MNRAS, 388, 500

Egusa, F., Kohno, K., Sofue, Y., Nakanishi, H., \& Komugi, S. 2009, ApJ, 697, 1870

Ferguson, A. M. N., Gallagher, J. S., \& Wyse, R. F. G. 1998, AJ, 116, 673

Foyle, K., Rix, H.-W., Walter, F., \& Leroy, A. K. 2010, ApJ, 725, 534

García-Benito, R., Díaz, A., Hägele, G. F., et al. 2010, MNRAS, 408, 2234

Garnett, D. R., \& Kennicutt, R. C. 1995, ApJ, 426, 123

Hodge, P. W. 1976, ApJ, 205, 728

Izotov, Y. I., Stasińska, G., Meynet, G., Guseva, N. G., \& Thuan, T. X. 2006, A\&A, 448, 955

James, P. A., \& Puxley, P. J. 1993, Nature, 363, 240

Kamphuis, J., \& Briggs, F. 1992, A\&A, 253, 335

Kennicutt, R. C. 1998, ARA\&A, 36, 189

Kennicutt, R. C., \& Hodge, P. W. 1976, ApJ, 205, 728

Kennicutt, R. C., \& Hodge, P. W. 1980, ApJ, 241, 573

Kennicutt, R. C., Bresolin, F., \& Garnett, D.R. 2003, ApJ, 591, 801

Kewley, L. J., \& Dopita, M. A. 2002, ApJS, 142, 35
Kobulnicky, H. A., \& Kewley, L. J. 2004, ApJ, 617, 240 (KK04)

McCall, M. L., Rybski, P. M., \& Shields, G.S. 1985, ApJS, 142, 35

Moustakas, J., Kennicutt, R. C. Jr., Tremonti, C. A., et al. 2010, AJS, 190, 233

López-Sánchez, Á. R., \& Esteban, C. 2010, A\&A, 517, A85

Lu, N. Y., Hoffman, G. L., Groff, T., Roos, T., \& Lamphier, C. 1993, ApJS, 88, 383

Oke, J. B. 1990, AJ, 99, 1621

Oke, J. B., \& Gunn, J. E. 1983, ApJ, 283, 158

Osterbrock, D. E., \& Ferland, G. J. 2006, Astrophysics of Gaseous Nebulae and Active Galactic Nuclei (University Science Books)

Pettini, M., \& Pagel, B. E. J. 2004, MNRAS, 348, 59

Pilyugin, L. S. 2001a, A\&A, 369, 594

Pilyugin, L. S. 2001b, A\&A, 373, 56

Pilyugin, L. S., \& Thuan, T. X. 2005, ApJ, 631, 231 (PT05)

Regan, M. W., \& Vogel, S. N. 1995, ApJ, 452, L21

Rosales-Ortega, F. F., Díaz, A. I., Kennicutt, R. C., \& Sánchez, S. F. 2011, MNRAS, 415, 2439 (RO11)

Sánchez, S. F., Rosales-Ortega, F. F., Kennicutt, R. C., et al. 2011, MNRAS, 410, 313

Schild, R. E. 1977, AJ, 82, 337

Searle, L. 1971, ApJ, 168, 327

Seaton, M. J. 1979, MNRAS, 187, 73

Sofue, Y., Tutui, Y., Honma, M., et al. 1999, ApJ, 523, 136

Talent, D. L. 1983, PASP, 95, 986

Tully, R. B. 1988, Nearby Galaxies Catalog (New York: Cambridge Univ. Press)

van Zee, L., Salzer, J. J., \& Haynes, M. P. 1998, ApJ, 497, 1

Zaritsky, D., Kennicutt, R. C., \& Huchra, J. P. 1994, ApJ, 420, 87

Pages 16 to 35 are available in the electronic edition of the journal at http://www . aanda. org 
Table 4. Positions and main properties of the H II regions of NGC 628. The H $\alpha$ fluxes are extinction corrected.

\begin{tabular}{|c|c|c|c|c|c|c|}
\hline Number & $X$ offset $(\operatorname{arcsec})$ & $Y$ offset $(\operatorname{arcsec})$ & Deprojected $R$ (arcmin) & $F_{\mathrm{H} \alpha}\left(10^{14} \mathrm{erg} / \mathrm{cm}^{2} / \mathrm{s}\right)$ & $A_{\mathrm{V}}(\mathrm{mag})$ & Area $\left(\operatorname{arcsec}^{2}\right)$ \\
\hline 1 & 87.49 & 183.08 & 3.39 & $14.78 \pm 1.45$ & $0.00 \pm 0.06$ & 36.2 \\
\hline 2 & 3.32 & 181.94 & 3.03 & $6.25 \pm 0.64$ & $0.00 \pm 0.06$ & 37.1 \\
\hline 3 & 14.15 & 176.81 & 2.96 & $1.55 \pm 0.18$ & $0.00 \pm 0.07$ & 9.9 \\
\hline 4 & 71.82 & 171.11 & 3.10 & $0.41 \pm 0.06$ & $0.00 \pm 0.09$ & 3.6 \\
\hline 5 & -43.51 & 153.92 & 2.67 & $7.86 \pm 0.79$ & $0.00 \pm 0.06$ & 18.6 \\
\hline 6 & 69.82 & 151.92 & 2.79 & $0.41 \pm 0.04$ & $0.79 \pm 0.11$ & 2.8 \\
\hline 7 & 167.96 & 145.75 & 3.72 & $1.61 \pm 0.18$ & $0.07 \pm 0.07$ & 8.3 \\
\hline 8 & 166.15 & 142.23 & 3.66 & $0.63 \pm 0.08$ & $0.15 \pm 0.09$ & 4.5 \\
\hline 9 & 145.25 & 138.15 & 3.36 & $1.16 \pm 0.14$ & $0.00 \pm 0.08$ & 8.4 \\
\hline 10 & 165.77 & 136.34 & 3.60 & $0.87 \pm 0.10$ & $0.32 \pm 0.08$ & 6.5 \\
\hline 11 & 41.61 & 132.92 & 2.32 & $5.78 \pm 0.50$ & $0.31 \pm 0.07$ & 24.4 \\
\hline 12 & 168.05 & 133.49 & 3.60 & $1.36 \pm 0.17$ & $0.00 \pm 0.07$ & 10.0 \\
\hline 13 & -41.99 & 134.06 & 2.34 & $0.74 \pm 0.10$ & $0.00 \pm 0.08$ & 5.9 \\
\hline 14 & -70.78 & 132.07 & 2.50 & $4.00 \pm 0.32$ & $0.49 \pm 0.07$ & 15.6 \\
\hline 15 & 38.57 & 129.88 & 2.26 & $0.31 \pm 0.04$ & $0.24 \pm 0.10$ & 2.4 \\
\hline 16 & 166.15 & 125.80 & 3.49 & $3.80 \pm 0.41$ & $0.00 \pm 0.07$ & 9.6 \\
\hline 17 & -98.52 & 124.56 & 2.65 & $1.95 \pm 0.23$ & $0.00 \pm 0.07$ & 14.6 \\
\hline 18 & 20.80 & 122.95 & 2.08 & $9.32 \pm 0.68$ & $0.51 \pm 0.07$ & 20.0 \\
\hline 19 & 42.75 & 124.18 & 2.19 & $0.41 \pm 0.06$ & $0.03 \pm 0.09$ & 2.2 \\
\hline 20 & 29.07 & 123.42 & 2.12 & $1.49 \pm 0.17$ & $0.14 \pm 0.08$ & 8.7 \\
\hline 21 & -73.72 & 118.86 & 2.33 & $12.81 \pm 1.27$ & $0.00 \pm 0.06$ & 73.5 \\
\hline 22 & 40.56 & 120.95 & 2.13 & $1.04 \pm 0.11$ & $0.35 \pm 0.08$ & 5.1 \\
\hline 23 & 172.71 & 119.15 & 3.52 & $1.92 \pm 0.22$ & $0.00 \pm 0.07$ & 7.6 \\
\hline 24 & 38.85 & 110.50 & 1.96 & $65.37 \pm 3.82$ & $0.67 \pm 0.06$ & 158.3 \\
\hline 25 & 27.36 & 117.44 & 2.01 & $2.00 \pm 0.16$ & $0.64 \pm 0.08$ & 7.7 \\
\hline 26 & 174.70 & 114.68 & 3.50 & $0.95 \pm 0.12$ & $0.00 \pm 0.08$ & 5.7 \\
\hline 27 & -61.85 & 111.83 & 2.13 & $9.47 \pm 0.87$ & $0.14 \pm 0.06$ & 31.9 \\
\hline 28 & 171.19 & 109.55 & 3.41 & $6.38 \pm 0.64$ & $0.05 \pm 0.06$ & 40.9 \\
\hline 29 & 13.11 & 111.36 & 1.87 & $3.48 \pm 0.37$ & $0.03 \pm 0.07$ & 12.2 \\
\hline 30 & -70.11 & 111.45 & 2.20 & $1.45 \pm 0.15$ & $0.31 \pm 0.08$ & 8.3 \\
\hline 31 & -50.83 & 109.74 & 2.02 & $0.69 \pm 0.06$ & $0.91 \pm 0.10$ & 2.9 \\
\hline 32 & -38.86 & 109.08 & 1.93 & $0.30 \pm 0.05$ & $0.13 \pm 0.10$ & 2.5 \\
\hline 33 & 12.73 & 104.80 & 1.76 & $15.35 \pm 1.37$ & $0.15 \pm 0.06$ & 44.7 \\
\hline 34 & -68.88 & 106.70 & 2.12 & $0.74 \pm 0.09$ & $0.20 \pm 0.09$ & 5.1 \\
\hline 35 & -17.96 & 107.75 & 1.82 & $0.31 \pm 0.04$ & $0.32 \pm 0.10$ & 2.1 \\
\hline 36 & -29.83 & 106.89 & 1.85 & $0.77 \pm 0.08$ & $0.40 \pm 0.09$ & 3.8 \\
\hline 37 & 20.04 & 106.80 & 1.81 & $0.43 \pm 0.06$ & $0.15 \pm 0.09$ & 2.7 \\
\hline 38 & -36.67 & 104.99 & 1.85 & $0.83 \pm 0.05$ & $1.85 \pm 0.12$ & 2.2 \\
\hline 39 & -94.53 & 99.96 & 2.30 & $2.19 \pm 0.15$ & $0.97 \pm 0.08$ & 9.3 \\
\hline 40 & -89.78 & 100.43 & 2.25 & $1.37 \pm 0.06$ & $1.98 \pm 0.11$ & 2.8 \\
\hline 41 & 8.55 & 97.58 & 1.63 & $5.34 \pm 0.36$ & $0.74 \pm 0.07$ & 22.5 \\
\hline 42 & -47.60 & 98.72 & 1.83 & $1.83 \pm 0.21$ & $0.00 \pm 0.07$ & 10.9 \\
\hline 43 & 126.06 & 98.44 & 2.68 & $1.59 \pm 0.15$ & $0.43 \pm 0.08$ & 8.2 \\
\hline 44 & 19.76 & 96.82 & 1.65 & $2.57 \pm 0.28$ & $0.09 \pm 0.07$ & 13.1 \\
\hline 45 & 51.49 & 91.88 & 1.76 & $0.95 \pm 0.07$ & $1.05 \pm 0.10$ & 3.1 \\
\hline 46 & 39.61 & 91.12 & 1.66 & $1.32 \pm 0.13$ & $0.45 \pm 0.08$ & 6.5 \\
\hline 47 & -127.02 & 89.32 & 2.60 & $3.64 \pm 0.32$ & $0.35 \pm 0.07$ & 18.2 \\
\hline 48 & 128.25 & 89.13 & 2.62 & $3.69 \pm 0.40$ & $0.00 \pm 0.07$ & 18.7 \\
\hline 49 & 43.79 & 89.60 & 1.67 & $1.39 \pm 0.13$ & $0.50 \pm 0.08$ & 7.2 \\
\hline 50 & 31.25 & 88.08 & 1.56 & $1.37 \pm 0.06$ & $2.40 \pm 0.12$ & 2.0 \\
\hline 51 & 56.90 & 82.10 & 1.67 & $14.33 \pm 0.95$ & $0.60 \pm 0.06$ & 40.4 \\
\hline 52 & -27.27 & 85.61 & 1.50 & $2.18 \pm 0.14$ & $1.05 \pm 0.08$ & 6.8 \\
\hline 53 & 51.87 & 83.81 & 1.65 & $0.84 \pm 0.07$ & $0.81 \pm 0.09$ & 3.9 \\
\hline 54 & 184.30 & 78.11 & 3.36 & $20.14 \pm 1.95$ & $0.00 \pm 0.06$ & 53.0 \\
\hline 55 & 121.12 & 81.72 & 2.45 & $1.01 \pm 0.07$ & $1.11 \pm 0.10$ & 3.6 \\
\hline 56 & -56.05 & 80.96 & 1.64 & $0.51 \pm 0.05$ & $0.61 \pm 0.10$ & 2.9 \\
\hline 57 & -17.58 & 77.92 & 1.33 & $8.37 \pm 0.82$ & $0.07 \pm 0.06$ & 32.1 \\
\hline 58 & -6.08 & 76.59 & 1.28 & $20.21 \pm 1.37$ & $0.54 \pm 0.06$ & 47.3 \\
\hline 59 & -132.34 & 77.92 & 2.57 & $2.93 \pm 0.19$ & $0.88 \pm 0.08$ & 12.6 \\
\hline 60 & 44.17 & 78.87 & 1.51 & $0.47 \pm 0.07$ & $0.04 \pm 0.09$ & 3.7 \\
\hline 61 & 21.94 & 77.54 & 1.34 & $1.53 \pm 0.17$ & $0.17 \pm 0.08$ & 6.0 \\
\hline 62 & -50.92 & 76.40 & 1.53 & $1.63 \pm 0.14$ & $0.59 \pm 0.08$ & 9.6 \\
\hline 63 & -28.60 & 72.03 & 1.29 & $4.71 \pm 0.39$ & $0.40 \pm 0.07$ & 23.5 \\
\hline 64 & -45.70 & 71.08 & 1.41 & $0.26 \pm 0.04$ & $0.14 \pm 0.10$ & 2.1 \\
\hline
\end{tabular}


Table 4. continued.

\begin{tabular}{|c|c|c|c|c|c|c|}
\hline Number & $X$ offset $(\operatorname{arcsec})$ & $Y$ offset $(\operatorname{arcsec})$ & Deprojected $R$ (arcmin) & $F_{\mathrm{H} \alpha}\left(10^{14} \mathrm{erg} / \mathrm{cm}^{2} / \mathrm{s}\right)$ & $A_{\mathrm{V}}(\mathrm{mag})$ & Area $\left(\operatorname{arcsec}^{2}\right)$ \\
\hline 65 & 129.39 & 67.09 & 2.44 & $9.41 \pm 0.95$ & $0.00 \pm 0.06$ & 33.4 \\
\hline 66 & 69.92 & 63.10 & 1.58 & $28.12 \pm 2.03$ & $0.42 \pm 0.06$ & 76.0 \\
\hline 67 & 83.50 & 67.09 & 1.79 & $0.42 \pm 0.05$ & $0.31 \pm 0.10$ & 2.5 \\
\hline 68 & -74.01 & 64.43 & 1.64 & $5.10 \pm 0.50$ & $0.13 \pm 0.07$ & 24.7 \\
\hline 69 & 2.56 & 65.47 & 1.09 & $0.96 \pm 0.09$ & $0.71 \pm 0.09$ & 4.1 \\
\hline 70 & 81.70 & 61.96 & 1.72 & $4.79 \pm 0.33$ & $0.73 \pm 0.07$ & 19.9 \\
\hline 71 & 100.89 & 61.10 & 1.98 & $2.03 \pm 0.14$ & $0.87 \pm 0.08$ & 8.4 \\
\hline 72 & 24.98 & 61.77 & 1.11 & $0.60 \pm 0.08$ & $0.18 \pm 0.09$ & 3.8 \\
\hline 73 & -29.26 & 58.06 & 1.08 & $0.40 \pm 0.05$ & $0.39 \pm 0.10$ & 2.3 \\
\hline 74 & 16.05 & 55.21 & 0.96 & $4.17 \pm 0.31$ & $0.62 \pm 0.07$ & 15.4 \\
\hline 75 & -46.84 & 56.92 & 1.23 & $2.13 \pm 0.16$ & $0.77 \pm 0.08$ & 6.7 \\
\hline 76 & -33.82 & 57.02 & 1.11 & $1.12 \pm 0.09$ & $0.96 \pm 0.09$ & 3.7 \\
\hline 77 & -22.99 & 56.83 & 1.02 & $0.57 \pm 0.05$ & $0.92 \pm 0.10$ & 2.8 \\
\hline 78 & 36.29 & 55.59 & 1.11 & $5.86 \pm 0.47$ & $0.44 \pm 0.07$ & 14.0 \\
\hline 79 & -86.07 & 55.88 & 1.72 & $1.29 \pm 0.11$ & $0.64 \pm 0.08$ & 5.3 \\
\hline 80 & 33.06 & 55.78 & 1.08 & $0.41 \pm 0.05$ & $0.61 \pm 0.11$ & 2.5 \\
\hline 81 & -15.30 & 53.03 & 0.92 & $0.78 \pm 0.06$ & $1.17 \pm 0.10$ & 3.0 \\
\hline 82 & -69.92 & 51.60 & 1.45 & $3.82 \pm 0.24$ & $0.93 \pm 0.07$ & 11.1 \\
\hline 83 & 95.95 & 51.51 & 1.83 & $0.25 \pm 0.04$ & $0.03 \pm 0.10$ & 2.2 \\
\hline 84 & -50.16 & 46.76 & 1.15 & $48.79 \pm 3.30$ & $0.48 \pm 0.06$ & 66.2 \\
\hline 85 & 110.29 & 49.04 & 2.02 & $1.30 \pm 0.07$ & $1.79 \pm 0.11$ & 3.0 \\
\hline 86 & 34.58 & 43.34 & 0.93 & $11.61 \pm 0.91$ & $0.38 \pm 0.06$ & 48.2 \\
\hline 87 & -82.84 & 44.48 & 1.57 & $248.45 \pm 3.91$ & $2.61 \pm 0.06$ & 38.5 \\
\hline 88 & 83.69 & 46.09 & 1.60 & $1.28 \pm 0.09$ & $1.13 \pm 0.09$ & 3.5 \\
\hline 89 & -113.53 & 41.91 & 2.03 & $0.80 \pm 0.07$ & $0.85 \pm 0.10$ & 3.4 \\
\hline 90 & 83.88 & 39.35 & 1.55 & $1.00 \pm 0.09$ & $0.63 \pm 0.09$ & 4.3 \\
\hline 91 & 31.44 & 36.21 & 0.80 & $1.02 \pm 0.07$ & $1.27 \pm 0.10$ & 3.5 \\
\hline 92 & -91.11 & 36.69 & 1.64 & $0.69 \pm 0.05$ & $1.49 \pm 0.11$ & 2.3 \\
\hline 93 & 86.35 & 32.89 & 1.55 & $5.10 \pm 0.33$ & $0.78 \pm 0.07$ & 20.4 \\
\hline 94 & -117.33 & 34.41 & 2.05 & $3.53 \pm 0.20$ & $1.09 \pm 0.08$ & 10.7 \\
\hline 95 & -49.88 & 33.46 & 1.00 & $0.39 \pm 0.06$ & $0.02 \pm 0.09$ & 2.9 \\
\hline 96 & 113.14 & 29.94 & 1.96 & $0.65 \pm 0.05$ & $1.22 \pm 0.11$ & 2.2 \\
\hline 97 & -9.69 & 28.52 & 0.50 & $0.51 \pm 0.05$ & $0.78 \pm 0.10$ & 2.3 \\
\hline 98 & 109.44 & 28.42 & 1.90 & $0.56 \pm 0.07$ & $0.38 \pm 0.09$ & 3.2 \\
\hline 99 & -120.75 & 27.76 & 2.08 & $2.35 \pm 0.11$ & $1.57 \pm 0.09$ & 6.4 \\
\hline 100 & -52.73 & 27.95 & 1.00 & $0.80 \pm 0.10$ & $0.16 \pm 0.08$ & 3.9 \\
\hline 101 & 44.46 & 25.00 & 0.86 & $9.33 \pm 0.72$ & $0.42 \pm 0.07$ & 33.1 \\
\hline 102 & -125.59 & 26.52 & 2.15 & $2.67 \pm 0.19$ & $0.74 \pm 0.08$ & 9.9 \\
\hline 103 & -30.97 & 24.53 & 0.66 & $0.49 \pm 0.05$ & $0.88 \pm 0.11$ & 2.2 \\
\hline 104 & -9.50 & 23.48 & 0.42 & $2.18 \pm 0.12$ & $1.42 \pm 0.09$ & 5.1 \\
\hline 105 & 38.76 & 21.77 & 0.75 & $0.74 \pm 0.06$ & $1.02 \pm 0.10$ & 3.0 \\
\hline 106 & 75.62 & 19.97 & 1.31 & $4.29 \pm 0.33$ & $0.57 \pm 0.07$ & 11.4 \\
\hline 107 & -132.24 & 17.88 & 2.24 & $8.56 \pm 0.58$ & $0.63 \pm 0.07$ & 19.8 \\
\hline 108 & 42.65 & 17.40 & 0.77 & $3.71 \pm 0.30$ & $0.51 \pm 0.07$ & 17.6 \\
\hline 109 & -38.57 & 16.17 & 0.70 & $0.67 \pm 0.08$ & $0.22 \pm 0.09$ & 3.5 \\
\hline 110 & 72.10 & 14.74 & 1.23 & $0.69 \pm 0.07$ & $0.66 \pm 0.09$ & 3.0 \\
\hline 111 & -133.76 & 14.17 & 2.25 & $0.48 \pm 0.04$ & $1.29 \pm 0.12$ & 2.4 \\
\hline 112 & -11.59 & 13.98 & 0.30 & $1.03 \pm 0.09$ & $0.83 \pm 0.09$ & 4.0 \\
\hline 113 & -85.22 & 11.42 & 1.44 & $4.32 \pm 0.33$ & $0.54 \pm 0.07$ & 18.5 \\
\hline 114 & 127.87 & 10.56 & 2.15 & $2.37 \pm 0.23$ & $0.29 \pm 0.07$ & 14.3 \\
\hline 115 & 9.31 & 12.27 & 0.26 & $0.59 \pm 0.07$ & $0.42 \pm 0.09$ & 2.2 \\
\hline 116 & 3.04 & 11.13 & 0.19 & $0.46 \pm 0.06$ & $0.17 \pm 0.09$ & 2.3 \\
\hline 117 & -34.39 & 10.75 & 0.60 & $1.59 \pm 0.13$ & $0.73 \pm 0.08$ & 5.4 \\
\hline 118 & 7.41 & 10.18 & 0.21 & $1.54 \pm 0.10$ & $1.22 \pm 0.09$ & 3.6 \\
\hline 119 & 112.48 & 7.90 & 1.89 & $1.92 \pm 0.17$ & $0.44 \pm 0.08$ & 8.3 \\
\hline 120 & 44.46 & 8.47 & 0.76 & $1.80 \pm 0.15$ & $0.60 \pm 0.08$ & 7.5 \\
\hline 121 & -75.34 & 6.76 & 1.27 & $2.19 \pm 0.12$ & $1.28 \pm 0.09$ & 5.9 \\
\hline 122 & -90.54 & 5.43 & 1.52 & $9.94 \pm 0.80$ & $0.34 \pm 0.06$ & 31.4 \\
\hline 123 & -9.79 & 6.76 & 0.20 & $1.15 \pm 0.07$ & $1.40 \pm 0.10$ & 2.7 \\
\hline 124 & -68.59 & 6.19 & 1.15 & $1.08 \pm 0.09$ & $0.87 \pm 0.09$ & 4.2 \\
\hline 125 & 48.92 & 1.54 & 0.82 & $5.74 \pm 0.45$ & $0.46 \pm 0.07$ & 23.8 \\
\hline 126 & 10.54 & 2.39 & 0.18 & $9.13 \pm 0.58$ & $0.74 \pm 0.07$ & 23.0 \\
\hline 127 & 98.99 & 4.58 & 1.66 & $0.69 \pm 0.06$ & $1.05 \pm 0.10$ & 3.2 \\
\hline 128 & 0.09 & -4.83 & 0.08 & $39.89 \pm 2.74$ & $0.48 \pm 0.06$ & 130.9 \\
\hline 129 & -43.23 & 3.82 & 0.73 & $0.90 \pm 0.07$ & $1.06 \pm 0.10$ & 2.9 \\
\hline
\end{tabular}


Table 4. continued.

\begin{tabular}{|c|c|c|c|c|c|c|}
\hline Number & $X$ offset $(\operatorname{arcsec})$ & $Y$ offset $(\operatorname{arcsec})$ & Deprojected $R$ (arcmin) & $F_{\mathrm{H} \alpha}\left(10^{14} \mathrm{erg} / \mathrm{cm}^{2} / \mathrm{s}\right)$ & $A_{\mathrm{V}}(\mathrm{mag})$ & Area $\left(\operatorname{arcsec}^{2}\right)$ \\
\hline 130 & 161.31 & 3.25 & 2.71 & $2.50 \pm 0.07$ & $3.00 \pm 0.12$ & 2.6 \\
\hline 131 & 77.99 & -0.46 & 1.31 & $2.83 \pm 0.17$ & $1.04 \pm 0.08$ & 10.0 \\
\hline 132 & -45.79 & 0.21 & 0.77 & $0.73 \pm 0.06$ & $1.02 \pm 0.10$ & 2.8 \\
\hline 133 & 64.31 & -0.27 & 1.08 & $0.47 \pm 0.05$ & $0.81 \pm 0.11$ & 2.3 \\
\hline 134 & 52.72 & -1.98 & 0.88 & $1.90 \pm 0.16$ & $0.59 \pm 0.08$ & 6.8 \\
\hline 135 & 157.79 & -3.12 & 2.65 & $1.75 \pm 0.19$ & $0.12 \pm 0.07$ & 7.7 \\
\hline 136 & -44.18 & -2.17 & 0.74 & $0.94 \pm 0.06$ & $1.48 \pm 0.11$ & 2.5 \\
\hline 137 & -47.22 & -2.83 & 0.79 & $0.76 \pm 0.05$ & $1.33 \pm 0.11$ & 2.3 \\
\hline 138 & 41.04 & -3.21 & 0.69 & $0.51 \pm 0.05$ & $0.74 \pm 0.10$ & 2.3 \\
\hline 139 & -11.40 & -7.58 & 0.23 & $7.11 \pm 0.34$ & $1.26 \pm 0.07$ & 17.6 \\
\hline 140 & -84.84 & -6.63 & 1.43 & $1.12 \pm 0.06$ & $1.82 \pm 0.11$ & 2.5 \\
\hline 141 & 96.14 & -8.44 & 1.62 & $0.68 \pm 0.05$ & $1.16 \pm 0.11$ & 2.2 \\
\hline 142 & -61.85 & -10.34 & 1.05 & $2.73 \pm 0.14$ & $1.38 \pm 0.08$ & 8.3 \\
\hline 143 & 88.44 & -10.91 & 1.49 & $2.28 \pm 0.14$ & $1.16 \pm 0.08$ & 6.5 \\
\hline 144 & 39.33 & -11.10 & 0.68 & $2.30 \pm 0.14$ & $1.19 \pm 0.08$ & 7.1 \\
\hline 145 & 86.64 & -13.66 & 1.47 & $2.36 \pm 0.11$ & $1.59 \pm 0.09$ & 4.9 \\
\hline 146 & -78.28 & -13.28 & 1.33 & $1.10 \pm 0.07$ & $1.32 \pm 0.10$ & 3.0 \\
\hline 147 & 18.62 & -14.14 & 0.39 & $0.70 \pm 0.07$ & $0.67 \pm 0.10$ & 3.2 \\
\hline 148 & 10.35 & -14.52 & 0.30 & $1.28 \pm 0.12$ & $0.48 \pm 0.08$ & 4.9 \\
\hline 149 & -100.80 & -17.08 & 1.71 & $1.13 \pm 0.07$ & $1.25 \pm 0.10$ & 2.6 \\
\hline 150 & 88.16 & -19.08 & 1.51 & $2.35 \pm 0.13$ & $1.36 \pm 0.09$ & 6.0 \\
\hline 151 & -42.47 & -20.88 & 0.79 & $5.30 \pm 0.48$ & $0.25 \pm 0.07$ & 18.4 \\
\hline 152 & 30.30 & -22.97 & 0.64 & $4.59 \pm 0.35$ & $0.56 \pm 0.07$ & 16.5 \\
\hline 153 & 143.16 & -23.26 & 2.43 & $0.78 \pm 0.05$ & $1.58 \pm 0.11$ & 2.5 \\
\hline 154 & -12.26 & -24.11 & 0.45 & $0.59 \pm 0.05$ & $1.03 \pm 0.11$ & 2.4 \\
\hline 155 & 73.43 & -28.77 & 1.32 & $22.45 \pm 1.54$ & $0.51 \pm 0.06$ & 48.4 \\
\hline 156 & -39.81 & -28.67 & 0.82 & $7.48 \pm 0.48$ & $0.75 \pm 0.07$ & 18.9 \\
\hline 157 & 25.27 & -28.96 & 0.64 & $4.09 \pm 0.25$ & $0.93 \pm 0.07$ & 12.6 \\
\hline 158 & 17.29 & -30.00 & 0.58 & $1.90 \pm 0.11$ & $1.37 \pm 0.09$ & 4.8 \\
\hline 159 & -48.74 & -32.57 & 0.98 & $4.25 \pm 0.34$ & $0.51 \pm 0.07$ & 17.9 \\
\hline 160 & 144.87 & -30.95 & 2.48 & $0.43 \pm 0.05$ & $0.47 \pm 0.10$ & 2.7 \\
\hline 161 & -10.74 & -32.19 & 0.57 & $0.88 \pm 0.07$ & $0.85 \pm 0.09$ & 3.4 \\
\hline 162 & -84.74 & -34.37 & 1.53 & $11.40 \pm 0.77$ & $0.61 \pm 0.06$ & 29.2 \\
\hline 163 & -95.29 & -32.95 & 1.69 & $0.67 \pm 0.05$ & $1.34 \pm 0.11$ & 2.2 \\
\hline 164 & 21.47 & -34.28 & 0.67 & $1.26 \pm 0.09$ & $1.10 \pm 0.09$ & 4.4 \\
\hline 165 & -10.26 & -34.75 & 0.60 & $0.77 \pm 0.05$ & $1.42 \pm 0.11$ & 2.3 \\
\hline 166 & 48.45 & -38.65 & 1.04 & $3.31 \pm 0.32$ & $0.22 \pm 0.07$ & 10.4 \\
\hline 167 & -88.73 & -43.02 & 1.65 & $3.66 \pm 0.18$ & $1.38 \pm 0.08$ & 8.4 \\
\hline 168 & 71.15 & -47.48 & 1.43 & $2.80 \pm 0.24$ & $0.47 \pm 0.07$ & 10.3 \\
\hline 169 & 9.21 & -48.24 & 0.82 & $3.58 \pm 0.26$ & $0.65 \pm 0.07$ & 13.0 \\
\hline 170 & 29.45 & -48.81 & 0.95 & $2.14 \pm 0.17$ & $0.62 \pm 0.08$ & 9.3 \\
\hline 171 & -113.15 & -48.34 & 2.06 & $0.86 \pm 0.06$ & $1.38 \pm 0.11$ & 2.9 \\
\hline 172 & -54.15 & -50.33 & 1.24 & $2.77 \pm 0.17$ & $1.01 \pm 0.08$ & 7.2 \\
\hline 173 & 57.38 & -50.05 & 1.27 & $0.81 \pm 0.06$ & $1.19 \pm 0.10$ & 2.6 \\
\hline 174 & -12.83 & -53.09 & 0.91 & $1.98 \pm 0.15$ & $0.77 \pm 0.08$ & 6.3 \\
\hline 175 & 43.22 & -55.46 & 1.17 & $3.76 \pm 0.14$ & $1.97 \pm 0.09$ & 5.2 \\
\hline 176 & -8.65 & -56.79 & 0.96 & $5.07 \pm 0.31$ & $0.90 \pm 0.07$ & 16.4 \\
\hline 177 & -133.76 & -58.03 & 2.45 & $1.12 \pm 0.06$ & $1.65 \pm 0.11$ & 3.0 \\
\hline 178 & 98.04 & -58.60 & 1.91 & $0.51 \pm 0.05$ & $0.90 \pm 0.11$ & 2.2 \\
\hline 179 & -1.62 & -60.78 & 1.01 & $1.48 \pm 0.10$ & $1.10 \pm 0.09$ & 4.0 \\
\hline 180 & -129.39 & -66.39 & 2.44 & $21.55 \pm 1.12$ & $0.93 \pm 0.06$ & 63.0 \\
\hline 181 & 7.31 & -61.45 & 1.03 & $0.95 \pm 0.06$ & $1.65 \pm 0.11$ & 2.3 \\
\hline 182 & 35.72 & -65.25 & 1.24 & $6.56 \pm 0.40$ & $0.86 \pm 0.07$ & 12.1 \\
\hline 183 & 31.54 & -63.63 & 1.18 & $3.34 \pm 0.09$ & $2.89 \pm 0.11$ & 3.3 \\
\hline 184 & 44.74 & -64.77 & 1.31 & $1.90 \pm 0.13$ & $1.01 \pm 0.08$ & 5.7 \\
\hline 185 & -22.90 & -66.20 & 1.17 & $2.54 \pm 0.19$ & $0.74 \pm 0.08$ & 11.0 \\
\hline 186 & 20.71 & -65.15 & 1.14 & $2.67 \pm 0.09$ & $2.49 \pm 0.11$ & 2.4 \\
\hline 187 & -78.66 & -72.47 & 1.79 & $28.19 \pm 1.54$ & $0.83 \pm 0.06$ & 69.7 \\
\hline 188 & -5.13 & -70.28 & 1.18 & $7.85 \pm 0.46$ & $0.91 \pm 0.07$ & 19.7 \\
\hline
\end{tabular}


B. Cedrés et al.: Two-dimensional metallicity distribution in NGC 628 and NGC 6946

Table 4. continued.

\begin{tabular}{|c|c|c|c|c|c|c|}
\hline Number & $X$ offset $(\operatorname{arcsec})$ & $Y$ offset $(\operatorname{arcsec})$ & Deprojected $R$ (arcmin) & $F_{\mathrm{H} \alpha}\left(10^{14} \mathrm{erg} / \mathrm{cm}^{2} / \mathrm{s}\right)$ & $A_{\mathrm{V}}(\mathrm{mag})$ & Area $\left(\operatorname{arcsec}^{2}\right)$ \\
\hline 189 & 122.93 & -71.23 & 2.38 & $0.83 \pm 0.05$ & $1.86 \pm 0.12$ & 2.1 \\
\hline 190 & 34.77 & -72.66 & 1.34 & $2.37 \pm 0.18$ & $0.68 \pm 0.08$ & 7.9 \\
\hline 191 & -54.72 & -73.70 & 1.54 & $1.36 \pm 0.06$ & $2.08 \pm 0.11$ & 2.3 \\
\hline 192 & 7.12 & -74.56 & 1.25 & $2.94 \pm 0.19$ & $0.91 \pm 0.08$ & 9.5 \\
\hline 193 & 32.20 & -77.79 & 1.40 & $11.80 \pm 0.73$ & $0.75 \pm 0.07$ & 28.6 \\
\hline 194 & 49.49 & -80.16 & 1.57 & $0.73 \pm 0.05$ & $1.54 \pm 0.11$ & 2.2 \\
\hline 195 & -81.13 & -83.01 & 1.94 & $1.04 \pm 0.06$ & $1.62 \pm 0.11$ & 2.6 \\
\hline 196 & -68.40 & -90.33 & 1.90 & $10.10 \pm 0.48$ & $1.17 \pm 0.07$ & 18.7 \\
\hline 197 & 105.45 & -91.66 & 2.33 & $1.11 \pm 0.09$ & $0.89 \pm 0.09$ & 4.8 \\
\hline 198 & 119.60 & -95.74 & 2.56 & $3.15 \pm 0.16$ & $1.31 \pm 0.08$ & 9.3 \\
\hline 199 & 62.03 & -95.65 & 1.90 & $1.13 \pm 0.06$ & $1.97 \pm 0.11$ & 2.5 \\
\hline 200 & -56.43 & -107.52 & 2.03 & $20.79 \pm 0.94$ & $1.16 \pm 0.06$ & 40.4 \\
\hline 201 & -20.81 & -107.43 & 1.83 & $2.36 \pm 0.12$ & $1.53 \pm 0.09$ & 6.0 \\
\hline 202 & 59.18 & -113.51 & 2.13 & $36.38 \pm 2.37$ & $0.55 \pm 0.06$ & 53.5 \\
\hline 203 & -10.26 & -112.75 & 1.89 & $5.92 \pm 0.37$ & $0.82 \pm 0.07$ & 15.5 \\
\hline 204 & -58.81 & -115.88 & 2.17 & $2.94 \pm 0.22$ & $0.69 \pm 0.07$ & 9.5 \\
\hline 205 & -40.09 & -124.81 & 2.19 & $67.67 \pm 3.60$ & $0.81 \pm 0.06$ & 144.3 \\
\hline 206 & -24.04 & -117.97 & 2.01 & $4.70 \pm 0.19$ & $1.67 \pm 0.08$ & 7.5 \\
\hline 207 & 50.73 & -123.20 & 2.22 & $6.96 \pm 0.31$ & $1.37 \pm 0.07$ & 15.8 \\
\hline 208 & 49.30 & -127.09 & 2.27 & $1.19 \pm 0.07$ & $1.56 \pm 0.10$ & 3.0 \\
\hline 209 & 78.66 & -132.41 & 2.57 & $2.91 \pm 0.09$ & $2.47 \pm 0.10$ & 5.6 \\
\hline
\end{tabular}


Table 5. Positions and main properties of the H II regions of NGC 6946. The H $\alpha$ fluxes are extinction-corrected.

\begin{tabular}{|c|c|c|c|c|c|c|}
\hline Number & $X$ offset $(\operatorname{arcsec})$ & $Y$ offset $(\operatorname{arcsec})$ & Deprojected $R$ (arcmin) & $F_{\mathrm{H} \alpha}\left(10^{14} \mathrm{erg} / \mathrm{cm}^{2} / \mathrm{s}\right)$ & $A_{\mathrm{V}}(\mathrm{mag})$ & Area $\left(\operatorname{arcsec}^{2}\right)$ \\
\hline 1 & 67.36 & 187.27 & 3.82 & $0.57 \pm 0.08$ & $0.09 \pm 0.09$ & 4.9 \\
\hline 2 & 111.44 & 171.78 & 3.93 & $0.60 \pm 0.07$ & $0.52 \pm 0.10$ & 5.3 \\
\hline 3 & 127.02 & 145.18 & 3.68 & $69.81 \pm 4.17$ & $0.66 \pm 0.06$ & 196.6 \\
\hline 4 & -59.09 & 154.11 & 2.96 & $0.68 \pm 0.06$ & $0.97 \pm 0.10$ & 3.6 \\
\hline 5 & 114.86 & 151.55 & 3.64 & $0.72 \pm 0.06$ & $1.14 \pm 0.11$ & 3.2 \\
\hline 6 & 66.69 & 147.65 & 3.12 & $2.68 \pm 0.15$ & $1.31 \pm 0.08$ & 8.8 \\
\hline 7 & 36.96 & 146.89 & 2.90 & $3.43 \pm 0.21$ & $1.01 \pm 0.08$ & 13.1 \\
\hline 8 & -56.33 & 144.33 & 2.77 & $2.99 \pm 0.28$ & $0.34 \pm 0.07$ & 20.2 \\
\hline 9 & 116.47 & 143.76 & 3.54 & $1.30 \pm 0.09$ & $1.16 \pm 0.09$ & 6.5 \\
\hline 10 & -62.79 & 138.72 & 2.70 & $6.21 \pm 0.33$ & $1.12 \pm 0.07$ & 10.2 \\
\hline 11 & -137.37 & 137.11 & 3.29 & $1.78 \pm 0.18$ & $0.34 \pm 0.08$ & 13.9 \\
\hline 12 & -122.45 & 136.25 & 3.12 & $0.64 \pm 0.08$ & $0.29 \pm 0.09$ & 5.3 \\
\hline 13 & 122.84 & 134.92 & 3.48 & $0.48 \pm 0.04$ & $1.19 \pm 0.12$ & 2.7 \\
\hline 14 & 138.51 & 131.22 & 3.61 & $8.61 \pm 0.49$ & $0.96 \pm 0.07$ & 27.6 \\
\hline 15 & 124.55 & 132.64 & 3.46 & $0.64 \pm 0.06$ & $0.97 \pm 0.10$ & 2.9 \\
\hline 16 & -138.79 & 130.27 & 3.22 & $0.52 \pm 0.05$ & $0.69 \pm 0.10$ & 3.3 \\
\hline 17 & 108.40 & 127.80 & 3.20 & $0.32 \pm 0.04$ & $0.71 \pm 0.12$ & 2.7 \\
\hline 18 & -112.48 & 119.91 & 2.80 & $28.81 \pm 2.35$ & $0.26 \pm 0.06$ & 117.6 \\
\hline 19 & 24.70 & 123.05 & 2.40 & $0.36 \pm 0.05$ & $0.41 \pm 0.11$ & 3.5 \\
\hline 20 & 142.60 & 122.00 & 3.54 & $0.58 \pm 0.06$ & $0.70 \pm 0.10$ & 4.8 \\
\hline 21 & -127.49 & 118.96 & 2.95 & $6.28 \pm 0.44$ & $0.65 \pm 0.07$ & 25.5 \\
\hline 22 & -91.86 & 120.10 & 2.60 & $5.47 \pm 0.40$ & $0.62 \pm 0.07$ & 15.1 \\
\hline 23 & 146.21 & 117.35 & 3.52 & $1.58 \pm 0.14$ & $0.61 \pm 0.08$ & 16.2 \\
\hline 24 & -140.31 & 118.01 & 3.09 & $2.34 \pm 0.20$ & $0.58 \pm 0.08$ & 9.7 \\
\hline 25 & 79.14 & 117.73 & 2.72 & $1.82 \pm 0.19$ & $0.25 \pm 0.08$ & 13.6 \\
\hline 26 & -99.65 & 117.35 & 2.63 & $4.56 \pm 0.26$ & $1.07 \pm 0.08$ & 12.1 \\
\hline 27 & -7.88 & 117.63 & 2.20 & $2.37 \pm 0.19$ & $0.69 \pm 0.08$ & 8.4 \\
\hline 28 & 156.75 & 114.02 & 3.63 & $2.68 \pm 0.17$ & $1.05 \pm 0.08$ & 11.3 \\
\hline 29 & -110.48 & 112.41 & 2.68 & $0.64 \pm 0.09$ & $0.07 \pm 0.09$ & 6.0 \\
\hline 30 & 123.69 & 112.41 & 3.16 & $0.56 \pm 0.06$ & $0.58 \pm 0.10$ & 4.6 \\
\hline 31 & -44.84 & 110.41 & 2.13 & $4.76 \pm 0.33$ & $0.71 \pm 0.07$ & 24.5 \\
\hline 32 & -137.94 & 111.08 & 2.98 & $1.18 \pm 0.14$ & $0.08 \pm 0.08$ & 8.4 \\
\hline 33 & -80.56 & 110.13 & 2.36 & $0.85 \pm 0.10$ & $0.32 \pm 0.09$ & 4.0 \\
\hline 34 & -93.95 & 102.34 & 2.37 & $100.99 \pm 5.16$ & $0.87 \pm 0.06$ & 273.1 \\
\hline 35 & 32.87 & 108.80 & 2.18 & $0.48 \pm 0.05$ & $0.69 \pm 0.10$ & 3.4 \\
\hline 36 & 158.46 & 106.52 & 3.56 & $2.07 \pm 0.12$ & $1.29 \pm 0.09$ & 7.5 \\
\hline 37 & -26.98 & 103.00 & 1.94 & $15.14 \pm 1.52$ & $0.00 \pm 0.06$ & 70.0 \\
\hline 38 & -147.34 & 102.24 & 3.00 & $15.41 \pm 1.08$ & $0.54 \pm 0.06$ & 66.8 \\
\hline 39 & -110.20 & 103.57 & 2.56 & $2.30 \pm 0.18$ & $0.74 \pm 0.08$ & 12.3 \\
\hline 40 & 30.69 & 102.05 & 2.04 & $4.29 \pm 0.31$ & $0.68 \pm 0.07$ & 18.9 \\
\hline 41 & -7.12 & 100.34 & 1.87 & $3.33 \pm 0.28$ & $0.49 \pm 0.07$ & 18.8 \\
\hline 42 & -35.81 & 96.73 & 1.85 & $10.07 \pm 1.01$ & $0.03 \pm 0.06$ & 64.5 \\
\hline 43 & -48.45 & 97.87 & 1.93 & $10.12 \pm 0.72$ & $0.57 \pm 0.07$ & 33.8 \\
\hline 44 & 137.37 & 100.34 & 3.18 & $1.08 \pm 0.07$ & $1.55 \pm 0.11$ & 4.2 \\
\hline 45 & -72.20 & 99.58 & 2.12 & $3.79 \pm 0.29$ & $0.63 \pm 0.07$ & 15.7 \\
\hline 46 & -138.13 & 99.68 & 2.85 & $0.37 \pm 0.05$ & $0.45 \pm 0.11$ & 3.1 \\
\hline 47 & -170.14 & 98.16 & 3.28 & $0.47 \pm 0.07$ & $0.00 \pm 0.09$ & 5.1 \\
\hline 48 & -114.47 & 94.74 & 2.50 & $16.32 \pm 0.82$ & $1.05 \pm 0.07$ & 37.6 \\
\hline 49 & -132.62 & 96.35 & 2.75 & $0.38 \pm 0.04$ & $0.67 \pm 0.11$ & 2.6 \\
\hline 50 & -126.73 & 93.22 & 2.64 & $1.26 \pm 0.12$ & $0.59 \pm 0.08$ & 5.5 \\
\hline 51 & 46.65 & 92.84 & 2.00 & $1.05 \pm 0.10$ & $0.54 \pm 0.09$ & 5.3 \\
\hline 52 & -11.78 & 86.57 & 1.62 & $7.82 \pm 0.82$ & $0.00 \pm 0.06$ & 53.5 \\
\hline 53 & -133.85 & 88.37 & 2.68 & $5.94 \pm 0.33$ & $1.07 \pm 0.07$ & 14.4 \\
\hline 54 & 32.97 & 86.09 & 1.77 & $4.34 \pm 0.47$ & $0.00 \pm 0.07$ & 33.2 \\
\hline 55 & -141.45 & 81.15 & 2.72 & $20.83 \pm 0.89$ & $1.29 \pm 0.07$ & 49.5 \\
\hline 56 & 37.34 & 84.00 & 1.77 & $0.98 \pm 0.11$ & $0.30 \pm 0.08$ & 6.6 \\
\hline 57 & 149.72 & 84.76 & 3.18 & $1.04 \pm 0.07$ & $1.42 \pm 0.10$ & 4.4 \\
\hline 58 & -150.86 & 79.35 & 2.84 & $0.74 \pm 0.06$ & $1.13 \pm 0.10$ & 3.0 \\
\hline 59 & 147.25 & 78.40 & 3.07 & $1.38 \pm 0.17$ & $0.00 \pm 0.08$ & 8.1 \\
\hline 60 & -11.40 & 76.40 & 1.43 & $7.59 \pm 0.61$ & $0.40 \pm 0.07$ & 32.6 \\
\hline 61 & -149.72 & 73.36 & 2.78 & $8.01 \pm 0.47$ & $0.91 \pm 0.07$ & 32.2 \\
\hline 62 & -28.97 & 74.50 & 1.43 & $0.30 \pm 0.04$ & $0.43 \pm 0.11$ & 2.6 \\
\hline 63 & 151.62 & 65.95 & 3.01 & $39.40 \pm 1.39$ & $1.51 \pm 0.06$ & 88.2 \\
\hline 64 & 159.32 & 70.13 & 3.17 & $4.66 \pm 0.25$ & $1.20 \pm 0.08$ & 10.4 \\
\hline
\end{tabular}


Table 5. continued.

\begin{tabular}{|c|c|c|c|c|c|c|}
\hline Number & $X$ offset $(\operatorname{arcsec})$ & $Y$ offset $(\operatorname{arcsec})$ & Deprojected $R$ (arcmin) & $F_{\mathrm{H} \alpha}\left(10^{14} \mathrm{erg} / \mathrm{cm}^{2} / \mathrm{s}\right)$ & $A_{\mathrm{V}}(\mathrm{mag})$ & Area $\left(\operatorname{arcsec}^{2}\right)$ \\
\hline 65 & 17.20 & 70.23 & 1.38 & $1.23 \pm 0.14$ & $0.23 \pm 0.08$ & 6.7 \\
\hline 66 & 10.74 & 67.85 & 1.31 & $8.63 \pm 0.82$ & $0.14 \pm 0.06$ & 19.4 \\
\hline 67 & -139.74 & 68.71 & 2.60 & $0.85 \pm 0.06$ & $1.44 \pm 0.11$ & 3.8 \\
\hline 68 & -0.85 & 65.57 & 1.23 & $0.97 \pm 0.11$ & $0.22 \pm 0.08$ & 6.1 \\
\hline 69 & 118.85 & 64.34 & 2.49 & $0.37 \pm 0.04$ & $0.70 \pm 0.11$ & 3.1 \\
\hline 70 & -86.92 & 63.20 & 1.80 & $1.32 \pm 0.11$ & $0.79 \pm 0.09$ & 6.2 \\
\hline 71 & -166.34 & 62.44 & 2.97 & $1.21 \pm 0.12$ & $0.51 \pm 0.08$ & 8.9 \\
\hline 72 & 157.70 & 63.29 & 3.08 & $2.09 \pm 0.11$ & $1.45 \pm 0.09$ & 4.2 \\
\hline 73 & -39.99 & 62.25 & 1.29 & $0.62 \pm 0.07$ & $0.35 \pm 0.09$ & 5.3 \\
\hline 74 & -23.65 & 59.11 & 1.14 & $0.74 \pm 0.10$ & $0.00 \pm 0.08$ & 8.4 \\
\hline 75 & -79.42 & 55.41 & 1.62 & $3.15 \pm 0.31$ & $0.23 \pm 0.07$ & 21.0 \\
\hline 76 & 32.68 & 56.07 & 1.25 & $0.35 \pm 0.06$ & $0.02 \pm 0.10$ & 3.8 \\
\hline 77 & 24.13 & 53.89 & 1.14 & $1.82 \pm 0.19$ & $0.21 \pm 0.08$ & 14.1 \\
\hline 78 & 67.45 & 52.75 & 1.61 & $5.35 \pm 0.44$ & $0.43 \pm 0.07$ & 13.5 \\
\hline 79 & -2.56 & 51.70 & 0.97 & $0.46 \pm 0.06$ & $0.33 \pm 0.10$ & 3.1 \\
\hline 80 & 85.03 & 45.05 & 1.77 & $6.58 \pm 0.37$ & $1.01 \pm 0.07$ & 20.4 \\
\hline 81 & -69.25 & 47.52 & 1.40 & $0.94 \pm 0.07$ & $1.04 \pm 0.10$ & 3.6 \\
\hline 82 & -16.72 & 46.95 & 0.90 & $0.38 \pm 0.06$ & $0.01 \pm 0.10$ & 3.9 \\
\hline 83 & 113.05 & 44.86 & 2.20 & $2.34 \pm 0.23$ & $0.26 \pm 0.07$ & 14.1 \\
\hline 84 & 12.73 & 43.53 & 0.87 & $3.67 \pm 0.36$ & $0.22 \pm 0.07$ & 19.8 \\
\hline 85 & -122.07 & 43.91 & 2.17 & $4.68 \pm 0.24$ & $1.27 \pm 0.08$ & 13.4 \\
\hline 86 & 41.61 & 43.06 & 1.14 & $0.65 \pm 0.07$ & $0.46 \pm 0.09$ & 6.1 \\
\hline 87 & 64.22 & 43.06 & 1.44 & $0.36 \pm 0.05$ & $0.24 \pm 0.10$ & 3.5 \\
\hline 88 & 36.86 & 43.06 & 1.08 & $0.42 \pm 0.05$ & $0.39 \pm 0.10$ & 3.0 \\
\hline 89 & -67.92 & 42.01 & 1.33 & $0.69 \pm 0.07$ & $0.59 \pm 0.09$ & 4.9 \\
\hline 90 & -88.35 & 41.16 & 1.62 & $1.63 \pm 0.13$ & $0.73 \pm 0.08$ & 6.8 \\
\hline 91 & -71.63 & 40.87 & 1.38 & $1.80 \pm 0.16$ & $0.59 \pm 0.08$ & 9.6 \\
\hline 92 & 8.65 & 39.16 & 0.77 & $1.78 \pm 0.21$ & $0.04 \pm 0.07$ & 12.4 \\
\hline 93 & 79.23 & 39.64 & 1.62 & $0.84 \pm 0.09$ & $0.36 \pm 0.09$ & 6.4 \\
\hline 94 & -42.94 & 37.17 & 0.96 & $1.70 \pm 0.21$ & $0.00 \pm 0.07$ & 11.6 \\
\hline 95 & -94.05 & 34.98 & 1.67 & $6.18 \pm 0.36$ & $0.98 \pm 0.07$ & 14.4 \\
\hline 96 & -123.69 & 32.13 & 2.14 & $5.85 \pm 0.39$ & $0.77 \pm 0.07$ & 24.9 \\
\hline 97 & 111.91 & 34.22 & 2.10 & $0.46 \pm 0.06$ & $0.24 \pm 0.10$ & 5.2 \\
\hline 98 & 8.27 & 27.19 & 0.55 & $18.59 \pm 1.29$ & $0.54 \pm 0.06$ & 67.5 \\
\hline 99 & -69.16 & 32.80 & 1.28 & $1.94 \pm 0.23$ & $0.05 \pm 0.07$ & 15.5 \\
\hline 100 & -48.26 & 31.85 & 0.97 & $1.24 \pm 0.11$ & $0.73 \pm 0.09$ & 6.6 \\
\hline 101 & -1.61 & 31.37 & 0.59 & $2.71 \pm 0.26$ & $0.31 \pm 0.07$ & 8.7 \\
\hline 102 & 43.80 & 30.71 & 1.00 & $0.19 \pm 0.03$ & $0.12 \pm 0.12$ & 2.6 \\
\hline 103 & -72.48 & 28.71 & 1.30 & $2.50 \pm 0.16$ & $1.08 \pm 0.08$ & 7.1 \\
\hline 104 & 109.63 & 28.33 & 2.02 & $0.80 \pm 0.07$ & $0.87 \pm 0.10$ & 4.4 \\
\hline 105 & 114.38 & 24.06 & 2.07 & $7.90 \pm 0.73$ & $0.19 \pm 0.07$ & 41.0 \\
\hline 106 & 70.21 & 25.48 & 1.35 & $0.22 \pm 0.04$ & $0.00 \pm 0.11$ & 3.2 \\
\hline 107 & -7.41 & 21.68 & 0.41 & $2.61 \pm 0.26$ & $0.25 \pm 0.07$ & 17.7 \\
\hline 108 & -14.34 & 22.16 & 0.46 & $2.88 \pm 0.22$ & $0.72 \pm 0.08$ & 11.0 \\
\hline 109 & 50.26 & 20.64 & 0.99 & $3.44 \pm 0.21$ & $1.01 \pm 0.08$ & 16.0 \\
\hline 110 & -95.95 & 21.49 & 1.65 & $1.26 \pm 0.10$ & $0.88 \pm 0.09$ & 5.4 \\
\hline 111 & 172.62 & 21.59 & 3.04 & $0.88 \pm 0.06$ & $1.25 \pm 0.10$ & 3.0 \\
\hline 112 & -114.38 & 19.97 & 1.96 & $1.99 \pm 0.11$ & $1.50 \pm 0.09$ & 6.6 \\
\hline 113 & -125.59 & 18.17 & 2.15 & $14.01 \pm 0.81$ & $0.86 \pm 0.07$ & 23.1 \\
\hline 114 & 111.82 & 18.64 & 1.99 & $0.60 \pm 0.08$ & $0.18 \pm 0.09$ & 6.9 \\
\hline 115 & -2.85 & 17.50 & 0.33 & $6.79 \pm 0.35$ & $1.16 \pm 0.07$ & 14.2 \\
\hline 116 & 26.32 & 17.31 & 0.59 & $0.98 \pm 0.11$ & $0.31 \pm 0.08$ & 7.1 \\
\hline 117 & -119.79 & 17.22 & 2.05 & $3.68 \pm 0.19$ & $1.31 \pm 0.08$ & 8.2 \\
\hline 118 & 2.57 & 6.01 & 0.13 & $52.66 \pm 4.11$ & $0.29 \pm 0.06$ & 179.4 \\
\hline 119 & -82.36 & 15.03 & 1.41 & $1.00 \pm 0.09$ & $0.79 \pm 0.09$ & 5.2 \\
\hline 120 & 65.46 & 15.32 & 1.19 & $1.10 \pm 0.10$ & $0.74 \pm 0.09$ & 7.1 \\
\hline 121 & -124.92 & 12.94 & 2.13 & $2.31 \pm 0.10$ & $1.95 \pm 0.10$ & 4.3 \\
\hline 122 & -0.38 & 12.85 & 0.24 & $0.34 \pm 0.04$ & $0.60 \pm 0.11$ & 2.6 \\
\hline 123 & -20.04 & 7.81 & 0.36 & $7.40 \pm 0.63$ & $0.32 \pm 0.07$ & 36.7 \\
\hline 124 & -75.90 & 10.95 & 1.30 & $1.92 \pm 0.20$ & $0.27 \pm 0.08$ & 13.6 \\
\hline 125 & 2.57 & 10.19 & 0.20 & $1.96 \pm 0.13$ & $1.04 \pm 0.08$ & 5.1 \\
\hline
\end{tabular}


Table 5. continued.

\begin{tabular}{|c|c|c|c|c|c|c|}
\hline Number & $X$ offset $(\operatorname{arcsec})$ & $Y$ offset $(\operatorname{arcsec})$ & Deprojected $R$ (arcmin) & $F_{\mathrm{H} \alpha}\left(10^{14} \mathrm{erg} / \mathrm{cm}^{2} / \mathrm{s}\right)$ & $A_{\mathrm{V}}(\mathrm{mag})$ & Area $\left(\operatorname{arcsec}^{2}\right)$ \\
\hline 126 & -130.15 & 10.38 & 2.22 & $1.35 \pm 0.06$ & $2.19 \pm 0.12$ & 2.6 \\
\hline 127 & 27.65 & 7.62 & 0.51 & $3.83 \pm 0.41$ & $0.06 \pm 0.07$ & 22.2 \\
\hline 128 & -7.50 & 9.14 & 0.20 & $0.94 \pm 0.09$ & $0.62 \pm 0.09$ & 4.4 \\
\hline 129 & -138.89 & 8.57 & 2.37 & $2.39 \pm 0.09$ & $2.20 \pm 0.10$ & 3.6 \\
\hline 130 & -134.90 & 6.20 & 2.31 & $13.75 \pm 0.60$ & $1.31 \pm 0.07$ & 26.6 \\
\hline 131 & 110.87 & 5.82 & 1.92 & $0.83 \pm 0.09$ & $0.32 \pm 0.09$ & 6.6 \\
\hline 132 & -9.69 & 5.63 & 0.19 & $3.38 \pm 0.28$ & $0.49 \pm 0.07$ & 10.8 \\
\hline 133 & 101.65 & -0.55 & 1.75 & $9.94 \pm 0.96$ & $0.10 \pm 0.06$ & 67.1 \\
\hline 134 & -135.66 & 1.45 & 2.33 & $1.55 \pm 0.10$ & $1.28 \pm 0.09$ & 4.1 \\
\hline 135 & -144.30 & -14.89 & 2.53 & $174.99 \pm 6.36$ & $1.35 \pm 0.06$ & 284.3 \\
\hline 136 & -129.20 & -0.64 & 2.22 & $1.83 \pm 0.10$ & $1.49 \pm 0.09$ & 6.1 \\
\hline 137 & 59.00 & -1.02 & 1.01 & $0.45 \pm 0.07$ & $0.00 \pm 0.09$ & 5.8 \\
\hline 138 & -100.32 & -5.39 & 1.74 & $0.81 \pm 0.05$ & $1.67 \pm 0.12$ & 2.6 \\
\hline 139 & -70.68 & -10.05 & 1.25 & $3.05 \pm 0.24$ & $0.63 \pm 0.07$ & 13.6 \\
\hline 140 & -66.31 & -13.18 & 1.19 & $1.05 \pm 0.11$ & $0.36 \pm 0.08$ & 9.4 \\
\hline 141 & 129.30 & -12.04 & 2.21 & $0.60 \pm 0.06$ & $0.82 \pm 0.10$ & 3.7 \\
\hline 142 & -4.08 & -14.04 & 0.28 & $3.43 \pm 0.20$ & $1.09 \pm 0.08$ & 9.0 \\
\hline 143 & -154.28 & -18.50 & 2.71 & $3.13 \pm 0.13$ & $1.93 \pm 0.09$ & 4.5 \\
\hline 144 & -160.17 & -22.21 & 2.83 & $56.51 \pm 1.01$ & $2.63 \pm 0.07$ & 51.2 \\
\hline 145 & -55.76 & -24.77 & 1.11 & $8.43 \pm 0.64$ & $0.50 \pm 0.07$ & 47.0 \\
\hline 146 & 132.72 & -23.25 & 2.27 & $9.84 \pm 0.54$ & $0.98 \pm 0.07$ & 23.6 \\
\hline 147 & -4.94 & -23.63 & 0.46 & $6.79 \pm 0.28$ & $1.55 \pm 0.08$ & 20.1 \\
\hline 148 & -16.05 & -21.83 & 0.52 & $1.10 \pm 0.06$ & $1.64 \pm 0.11$ & 3.6 \\
\hline 149 & -13.39 & -24.20 & 0.53 & $1.88 \pm 0.12$ & $1.08 \pm 0.09$ & 9.0 \\
\hline 150 & -44.08 & -35.79 & 1.07 & $3.81 \pm 0.33$ & $0.40 \pm 0.07$ & 23.1 \\
\hline 151 & -19.00 & -35.22 & 0.77 & $1.38 \pm 0.09$ & $1.13 \pm 0.09$ & 4.0 \\
\hline 152 & 121.89 & -35.60 & 2.13 & $2.05 \pm 0.14$ & $1.02 \pm 0.08$ & 5.4 \\
\hline 153 & -148.48 & -38.26 & 2.73 & $6.06 \pm 0.24$ & $1.65 \pm 0.08$ & 14.2 \\
\hline 154 & 113.24 & -38.36 & 2.00 & $12.28 \pm 0.43$ & $1.71 \pm 0.07$ & 12.8 \\
\hline 155 & -2.94 & -40.26 & 0.76 & $1.94 \pm 0.20$ & $0.24 \pm 0.08$ & 17.8 \\
\hline 156 & -56.90 & -40.92 & 1.31 & $2.73 \pm 0.20$ & $0.78 \pm 0.08$ & 12.7 \\
\hline 157 & 70.30 & -41.68 & 1.36 & $0.91 \pm 0.06$ & $1.33 \pm 0.10$ & 4.1 \\
\hline 158 & -43.98 & -43.77 & 1.18 & $2.47 \pm 0.15$ & $1.08 \pm 0.08$ & 7.6 \\
\hline 159 & 160.84 & -46.05 & 2.80 & $18.86 \pm 0.68$ & $1.58 \pm 0.07$ & 39.7 \\
\hline 160 & 86.64 & -43.77 & 1.62 & $1.33 \pm 0.13$ & $0.49 \pm 0.08$ & 7.7 \\
\hline 161 & 80.56 & -43.58 & 1.53 & $0.50 \pm 0.05$ & $0.71 \pm 0.10$ & 3.5 \\
\hline 162 & -137.75 & -44.06 & 2.60 & $1.83 \pm 0.07$ & $2.31 \pm 0.11$ & 3.5 \\
\hline 163 & -33.25 & -47.10 & 1.11 & $8.87 \pm 0.40$ & $1.35 \pm 0.07$ & 19.9 \\
\hline 164 & -133.66 & -45.86 & 2.54 & $4.01 \pm 0.13$ & $2.36 \pm 0.09$ & 6.5 \\
\hline 165 & -47.88 & -46.53 & 1.27 & $0.59 \pm 0.06$ & $0.71 \pm 0.10$ & 3.5 \\
\hline 166 & -58.80 & -49.95 & 1.45 & $3.16 \pm 0.19$ & $1.05 \pm 0.08$ & 14.4 \\
\hline 167 & 20.81 & -48.52 & 0.94 & $6.40 \pm 0.30$ & $1.33 \pm 0.07$ & 15.7 \\
\hline 168 & -65.26 & -50.71 & 1.55 & $2.92 \pm 0.14$ & $1.51 \pm 0.09$ & 6.2 \\
\hline 169 & 28.79 & -54.70 & 1.09 & $7.49 \pm 0.56$ & $0.52 \pm 0.07$ & 37.6 \\
\hline 170 & -39.52 & -52.04 & 1.25 & $0.86 \pm 0.06$ & $1.49 \pm 0.11$ & 4.0 \\
\hline 171 & 121.60 & -57.45 & 2.24 & $22.17 \pm 1.11$ & $1.03 \pm 0.06$ & 64.8 \\
\hline 172 & 50.54 & -54.51 & 1.27 & $2.05 \pm 0.18$ & $0.55 \pm 0.08$ & 10.5 \\
\hline 173 & 132.24 & -54.51 & 2.39 & $0.81 \pm 0.06$ & $1.11 \pm 0.10$ & 3.1 \\
\hline 174 & 157.89 & -56.50 & 2.80 & $1.15 \pm 0.15$ & $0.00 \pm 0.08$ & 9.6 \\
\hline 175 & 56.34 & -57.45 & 1.37 & $3.60 \pm 0.25$ & $0.80 \pm 0.07$ & 13.7 \\
\hline 176 & 136.04 & -58.40 & 2.47 & $2.55 \pm 0.14$ & $1.38 \pm 0.09$ & 9.2 \\
\hline 177 & 110.77 & -59.45 & 2.10 & $8.51 \pm 0.49$ & $0.92 \pm 0.07$ & 20.7 \\
\hline 178 & 102.32 & -60.02 & 1.98 & $1.66 \pm 0.13$ & $0.86 \pm 0.08$ & 6.8 \\
\hline 179 & -36.00 & -61.82 & 1.38 & $3.29 \pm 0.24$ & $0.75 \pm 0.08$ & 10.4 \\
\hline 180 & 160.84 & -64.67 & 2.89 & $5.87 \pm 0.31$ & $1.18 \pm 0.07$ & 21.2 \\
\hline 181 & -12.82 & -63.25 & 1.23 & $1.91 \pm 0.12$ & $1.18 \pm 0.09$ & 4.5 \\
\hline 182 & -34.86 & -66.00 & 1.44 & $1.74 \pm 0.12$ & $1.07 \pm 0.09$ & 6.8 \\
\hline 183 & -25.65 & -67.14 & 1.38 & $1.02 \pm 0.10$ & $0.46 \pm 0.09$ & 8.2 \\
\hline 184 & -21.28 & -68.09 & 1.37 & $1.93 \pm 0.12$ & $1.16 \pm 0.09$ & 7.0 \\
\hline 185 & -15.29 & -70.75 & 1.38 & $8.31 \pm 0.57$ & $0.64 \pm 0.07$ & 30.1 \\
\hline 186 & 147.16 & -71.13 & 2.72 & $6.17 \pm 0.48$ & $0.50 \pm 0.07$ & 36.9 \\
\hline
\end{tabular}


B. Cedrés et al.: Two-dimensional metallicity distribution in NGC 628 and NGC 6946

Table 5. continued.

\begin{tabular}{|c|c|c|c|c|c|c|}
\hline Number & $X$ offset (arcsec) & $Y$ offset $(\operatorname{arcsec})$ & Deprojected $R$ (arcmin) & $F_{\mathrm{H} \alpha}\left(10^{14} \mathrm{erg} / \mathrm{cm}^{2} / \mathrm{s}\right)$ & $A_{\mathrm{V}}(\mathrm{mag})$ & Area $\left(\operatorname{arcsec}^{2}\right)$ \\
\hline 187 & 112.58 & -71.51 & 2.23 & $1.22 \pm 0.10$ & $0.76 \pm 0.09$ & 4.3 \\
\hline 188 & -115.14 & -72.84 & 2.53 & $0.65 \pm 0.05$ & $1.53 \pm 0.12$ & 3.0 \\
\hline 189 & 24.04 & -74.36 & 1.41 & $8.59 \pm 0.62$ & $0.56 \pm 0.07$ & 25.7 \\
\hline 190 & 45.60 & -76.36 & 1.55 & $2.09 \pm 0.14$ & $0.99 \pm 0.08$ & 7.5 \\
\hline 191 & 35.25 & -82.25 & 1.59 & $12.53 \pm 0.95$ & $0.43 \pm 0.06$ & 54.8 \\
\hline 192 & -32.11 & -79.78 & 1.65 & $3.06 \pm 0.15$ & $1.55 \pm 0.09$ & 7.2 \\
\hline 193 & -4.27 & -81.49 & 1.54 & $4.49 \pm 0.49$ & $0.00 \pm 0.07$ & 16.6 \\
\hline 194 & 24.04 & -81.39 & 1.54 & $2.11 \pm 0.19$ & $0.46 \pm 0.08$ & 9.2 \\
\hline 195 & -125.87 & -82.53 & 2.80 & $1.12 \pm 0.06$ & $1.91 \pm 0.11$ & 2.7 \\
\hline 196 & -122.83 & -84.15 & 2.78 & $2.94 \pm 0.11$ & $2.13 \pm 0.10$ & 5.9 \\
\hline 197 & 14.06 & -87.57 & 1.63 & $12.80 \pm 0.86$ & $0.63 \pm 0.07$ & 49.6 \\
\hline 198 & -8.45 & -87.38 & 1.66 & $5.16 \pm 0.31$ & $0.97 \pm 0.07$ & 16.0 \\
\hline 199 & -77.80 & -86.81 & 2.22 & $0.86 \pm 0.05$ & $1.65 \pm 0.11$ & 4.3 \\
\hline 200 & 76.00 & -88.99 & 2.00 & $0.66 \pm 0.06$ & $0.88 \pm 0.10$ & 2.8 \\
\hline 201 & -16.91 & -95.36 & 1.85 & $17.62 \pm 1.01$ & $0.84 \pm 0.06$ & 46.5 \\
\hline 202 & -7.88 & -94.50 & 1.79 & $0.74 \pm 0.07$ & $0.76 \pm 0.10$ & 4.2 \\
\hline 203 & -11.21 & -95.93 & 1.83 & $0.50 \pm 0.05$ & $0.75 \pm 0.11$ & 2.6 \\
\hline 204 & -123.59 & -97.83 & 2.96 & $1.83 \pm 0.08$ & $2.09 \pm 0.11$ & 3.1 \\
\hline 205 & 3.90 & -106.57 & 1.99 & $15.97 \pm 0.78$ & $1.11 \pm 0.07$ & 49.0 \\
\hline 206 & 18.24 & -105.71 & 1.97 & $2.72 \pm 0.19$ & $0.88 \pm 0.08$ & 9.6 \\
\hline 207 & -111.53 & -109.23 & 2.96 & $16.10 \pm 0.66$ & $1.39 \pm 0.07$ & 51.9 \\
\hline 208 & -10.54 & -107.61 & 2.05 & $0.92 \pm 0.07$ & $1.20 \pm 0.10$ & 2.9 \\
\hline 209 & 24.89 & -111.51 & 2.09 & $2.32 \pm 0.19$ & $0.58 \pm 0.08$ & 11.8 \\
\hline 210 & -24.51 & -112.27 & 2.20 & $11.67 \pm 0.36$ & $1.97 \pm 0.07$ & 13.5 \\
\hline 211 & 42.94 & -113.79 & 2.18 & $8.91 \pm 0.32$ & $1.78 \pm 0.08$ & 18.3 \\
\hline 212 & -115.61 & -111.60 & 3.05 & $1.21 \pm 0.05$ & $2.30 \pm 0.12$ & 3.2 \\
\hline 213 & 80.18 & -112.84 & 2.39 & $4.51 \pm 0.23$ & $1.26 \pm 0.08$ & 14.0 \\
\hline 214 & -5.13 & -119.49 & 2.26 & $42.44 \pm 1.72$ & $1.28 \pm 0.06$ & 99.0 \\
\hline 215 & 30.50 & -112.93 & 2.13 & $0.72 \pm 0.09$ & $0.12 \pm 0.09$ & 4.5 \\
\hline 216 & 51.02 & -113.50 & 2.21 & $1.62 \pm 0.10$ & $1.19 \pm 0.09$ & 7.5 \\
\hline 217 & 146.11 & -113.69 & 3.11 & $0.87 \pm 0.05$ & $1.85 \pm 0.12$ & 2.6 \\
\hline 218 & 112.58 & -116.73 & 2.76 & $2.15 \pm 0.15$ & $0.94 \pm 0.08$ & 7.3 \\
\hline 219 & 146.02 & -117.02 & 3.14 & $0.79 \pm 0.05$ & $1.77 \pm 0.12$ & 3.4 \\
\hline 220 & 166.63 & -119.77 & 3.44 & $1.88 \pm 0.07$ & $2.50 \pm 0.12$ & 3.8 \\
\hline 221 & -26.03 & -123.86 & 2.42 & $2.23 \pm 0.11$ & $1.67 \pm 0.09$ & 6.0 \\
\hline 222 & -28.59 & -129.56 & 2.54 & $2.97 \pm 0.09$ & $2.66 \pm 0.11$ & 5.9 \\
\hline 223 & 45.60 & -135.35 & 2.57 & $3.23 \pm 0.10$ & $2.66 \pm 0.11$ & 4.5 \\
\hline 224 & 24.80 & -138.20 & 2.58 & $2.28 \pm 0.14$ & $1.20 \pm 0.08$ & 9.0 \\
\hline 225 & 31.83 & -143.81 & 2.69 & $19.92 \pm 0.63$ & $1.80 \pm 0.07$ & 37.1 \\
\hline 226 & -88.82 & -149.70 & 3.35 & $7.11 \pm 0.20$ & $2.33 \pm 0.08$ & 11.8 \\
\hline
\end{tabular}


Table 6. Equivalent width, $\mathrm{H} \beta$ flux and oxygen flux ratios, both extinction corrected, for all the H II regions of NGC 628 .

\begin{tabular}{|c|c|c|c|c|}
\hline Number & $\log (\mathrm{EWH} \alpha)(\AA)$ & $F_{\mathrm{H} \beta}\left(10^{14} \mathrm{erg} / \mathrm{cm}^{2} / \mathrm{s}\right)$ & $\log ([\mathrm{OII}] / \mathrm{H} \beta)$ & $\log ([\mathrm{OIII}] / \mathrm{H} \beta)$ \\
\hline 1 & $2.50 \pm 0.07$ & $6.04 \pm 1.35$ & $0.50 \pm 0.10$ & $0.26 \pm 0.10$ \\
\hline 2 & $2.34 \pm 0.08$ & $3.03 \pm 0.71$ & $0.47 \pm 0.11$ & $0.08 \pm 0.10$ \\
\hline 3 & $2.52 \pm 0.09$ & $0.64 \pm 0.17$ & $0.48 \pm 0.14$ & $0.12 \pm 0.12$ \\
\hline 4 & $2.83 \pm 0.11$ & $0.18 \pm 0.06$ & - & - \\
\hline 5 & $0.53 \pm 0.07$ & $4.80 \pm 0.99$ & - & - \\
\hline 6 & $2.34 \pm 0.08$ & $0.14 \pm 0.03$ & - & - \\
\hline 7 & $2.69 \pm 0.09$ & $0.56 \pm 0.14$ & $0.38 \pm 0.14$ & $0.22 \pm 0.12$ \\
\hline 8 & $2.53 \pm 0.10$ & $0.22 \pm 0.06$ & - & - \\
\hline 9 & $2.25 \pm 0.09$ & $0.52 \pm 0.14$ & $0.31 \pm 0.16$ & $0.19 \pm 0.13$ \\
\hline 10 & $1.83 \pm 0.08$ & $0.30 \pm 0.07$ & - & - \\
\hline 11 & $1.81 \pm 0.06$ & $2.01 \pm 0.36$ & $0.09 \pm 0.09$ & $-0.26 \pm 0.08$ \\
\hline 12 & $2.37 \pm 0.09$ & $0.50 \pm 0.14$ & $0.53 \pm 0.14$ & $0.11 \pm 0.13$ \\
\hline 13 & $2.43 \pm 0.10$ & $0.27 \pm 0.08$ & - & - \\
\hline 14 & $2.29 \pm 0.06$ & $1.38 \pm 0.22$ & $0.33 \pm 0.08$ & $-0.31 \pm 0.08$ \\
\hline 15 & $2.70 \pm 0.11$ & $0.11 \pm 0.03$ & - & - \\
\hline 16 & $0.64 \pm 0.08$ & $1.34 \pm 0.28$ & - & - \\
\hline 17 & $2.50 \pm 0.09$ & $0.68 \pm 0.18$ & $0.38 \pm 0.14$ & $-0.42 \pm 0.14$ \\
\hline 18 & $2.55 \pm 0.05$ & $3.22 \pm 0.47$ & $0.35 \pm 0.07$ & $-0.02 \pm 0.07$ \\
\hline 19 & $2.24 \pm 0.11$ & $0.14 \pm 0.05$ & - & - \\
\hline 20 & $2.20 \pm 0.08$ & $0.52 \pm 0.13$ & $0.39 \pm 0.14$ & $-0.38 \pm 0.13$ \\
\hline 21 & $1.96 \pm 0.07$ & $4.53 \pm 1.02$ & $0.20 \pm 0.10$ & $-0.08 \pm 0.10$ \\
\hline 22 & $2.69 \pm 0.08$ & $0.36 \pm 0.08$ & - & - \\
\hline 23 & $2.38 \pm 0.09$ & $0.75 \pm 0.20$ & $0.40 \pm 0.14$ & $0.20 \pm 0.12$ \\
\hline 24 & $2.33 \pm 0.04$ & $22.46 \pm 2.58$ & $0.26 \pm 0.05$ & $-0.40 \pm 0.05$ \\
\hline 25 & $2.46 \pm 0.06$ & $0.69 \pm 0.11$ & $0.26 \pm 0.10$ & $-0.59 \pm 0.10$ \\
\hline 26 & $2.21 \pm 0.09$ & $0.37 \pm 0.10$ & $0.45 \pm 0.17$ & $0.43 \pm 0.13$ \\
\hline 27 & $2.46 \pm 0.07$ & $3.30 \pm 0.67$ & $0.35 \pm 0.09$ & $-0.31 \pm 0.09$ \\
\hline 28 & $2.43 \pm 0.07$ & $2.23 \pm 0.50$ & $0.44 \pm 0.11$ & $0.13 \pm 0.10$ \\
\hline 29 & $2.43 \pm 0.08$ & $1.22 \pm 0.29$ & $0.35 \pm 0.12$ & $-0.19 \pm 0.11$ \\
\hline 30 & $2.28 \pm 0.08$ & $0.50 \pm 0.10$ & - & - \\
\hline 31 & $2.43 \pm 0.07$ & $0.24 \pm 0.04$ & - & - \\
\hline 32 & $2.04 \pm 0.11$ & $0.10 \pm 0.03$ & - & - \\
\hline 33 & $2.13 \pm 0.07$ & $5.35 \pm 1.04$ & $0.25 \pm 0.09$ & $-0.23 \pm 0.09$ \\
\hline 34 & $2.23 \pm 0.09$ & $0.26 \pm 0.06$ & - & - \\
\hline 35 & $2.14 \pm 0.10$ & $0.11 \pm 0.03$ & - & - \\
\hline 36 & $2.54 \pm 0.08$ & $0.26 \pm 0.06$ & - & - \\
\hline 37 & $3.25 \pm 0.11$ & $0.15 \pm 0.04$ & - & - \\
\hline 38 & $1.81 \pm 0.04$ & $0.28 \pm 0.02$ & $0.53 \pm 0.07$ & - \\
\hline 39 & $2.36 \pm 0.05$ & $0.75 \pm 0.09$ & $0.30 \pm 0.08$ & $-0.52 \pm 0.08$ \\
\hline 40 & $3.20 \pm 0.04$ & $0.46 \pm 0.03$ & - & - \\
\hline 41 & $1.90 \pm 0.05$ & $1.83 \pm 0.23$ & $-0.19 \pm 0.09$ & $-0.61 \pm 0.07$ \\
\hline 42 & $2.35 \pm 0.09$ & $0.74 \pm 0.19$ & - & - \\
\hline 43 & $2.53 \pm 0.07$ & $0.55 \pm 0.10$ & $0.58 \pm 0.10$ & $-0.05 \pm 0.09$ \\
\hline 44 & $2.23 \pm 0.08$ & $0.90 \pm 0.21$ & $0.13 \pm 0.14$ & $-0.62 \pm 0.13$ \\
\hline 45 & $2.21 \pm 0.06$ & $0.32 \pm 0.04$ & - & - \\
\hline 46 & $2.88 \pm 0.07$ & $0.45 \pm 0.09$ & - & - \\
\hline 47 & $2.32 \pm 0.06$ & $1.26 \pm 0.23$ & $0.33 \pm 0.09$ & $-0.29 \pm 0.09$ \\
\hline 48 & $2.56 \pm 0.08$ & $1.36 \pm 0.33$ & $0.39 \pm 0.12$ & $-0.08 \pm 0.11$ \\
\hline 49 & $3.21 \pm 0.07$ & $0.48 \pm 0.09$ & - & - \\
\hline 50 & $1.36 \pm 0.03$ & $0.45 \pm 0.03$ & $0.54 \pm 0.05$ & - \\
\hline 51 & $2.40 \pm 0.05$ & $4.93 \pm 0.64$ & $0.29 \pm 0.06$ & $-0.30 \pm 0.06$ \\
\hline 52 & $2.22 \pm 0.05$ & $0.74 \pm 0.08$ & $0.15 \pm 0.09$ & $-0.36 \pm 0.07$ \\
\hline 53 & $2.24 \pm 0.07$ & $0.29 \pm 0.04$ & - & - \\
\hline 54 & $2.45 \pm 0.07$ & $7.16 \pm 1.58$ & $0.57 \pm 0.10$ & $0.15 \pm 0.10$ \\
\hline 55 & $0.76 \pm 0.05$ & $0.34 \pm 0.04$ & - & - \\
\hline 56 & $2.24 \pm 0.08$ & $0.17 \pm 0.03$ & - & - \\
\hline 57 & $2.19 \pm 0.07$ & $2.92 \pm 0.63$ & $-0.23 \pm 0.12$ & $-0.80 \pm 0.11$ \\
\hline 58 & $2.23 \pm 0.05$ & $6.97 \pm 0.94$ & $0.11 \pm 0.06$ & $-0.61 \pm 0.06$ \\
\hline 59 & $2.07 \pm 0.05$ & $1.00 \pm 0.12$ & $0.33 \pm 0.07$ & $-0.80 \pm 0.09$ \\
\hline 60 & $2.44 \pm 0.11$ & $0.16 \pm 0.05$ & - & - \\
\hline 61 & $2.47 \pm 0.08$ & $0.53 \pm 0.12$ & - & - \\
\hline 62 & $1.92 \pm 0.06$ & $0.56 \pm 0.09$ & - & - \\
\hline 63 & $2.19 \pm 0.06$ & $1.63 \pm 0.28$ & $-0.11 \pm 0.11$ & $-1.04 \pm 0.11$ \\
\hline
\end{tabular}


Table 6. continued.

\begin{tabular}{|c|c|c|c|c|}
\hline Number & $\log (\mathrm{EWH} \alpha)(\AA)$ & $F_{\mathrm{H} \beta}\left(10^{14} \mathrm{erg} / \mathrm{cm}^{2} / \mathrm{s}\right)$ & $\log ([\mathrm{OII}] / \mathrm{H} \beta)$ & $\log ([\mathrm{OIII}] / \mathrm{H} \beta)$ \\
\hline 64 & $2.49 \pm 0.12$ & $0.09 \pm 0.03$ & - & - \\
\hline 65 & $2.13 \pm 0.07$ & $3.42 \pm 0.78$ & $0.36 \pm 0.11$ & $-0.13 \pm 0.10$ \\
\hline 66 & $2.26 \pm 0.05$ & $9.73 \pm 1.44$ & $0.12 \pm 0.07$ & $-0.60 \pm 0.07$ \\
\hline 67 & $2.22 \pm 0.10$ & $0.15 \pm 0.04$ & - & - \\
\hline 68 & $2.15 \pm 0.07$ & $1.78 \pm 0.38$ & $0.01 \pm 0.12$ & $-0.70 \pm 0.11$ \\
\hline 69 & $2.29 \pm 0.07$ & $0.33 \pm 0.05$ & - & - \\
\hline 70 & $1.97 \pm 0.05$ & $1.64 \pm 0.21$ & $-0.16 \pm 0.09$ & $-0.69 \pm 0.07$ \\
\hline 71 & $2.05 \pm 0.05$ & $0.69 \pm 0.09$ & $0.31 \pm 0.09$ & $-0.40 \pm 0.08$ \\
\hline 72 & $1.89 \pm 0.09$ & $0.21 \pm 0.06$ & - & - \\
\hline 73 & $2.48 \pm 0.09$ & $0.14 \pm 0.03$ & - & - \\
\hline 74 & $2.11 \pm 0.05$ & $1.44 \pm 0.20$ & - & - \\
\hline 75 & $2.40 \pm 0.06$ & $0.73 \pm 0.10$ & - & - \\
\hline 76 & $2.34 \pm 0.06$ & $0.38 \pm 0.05$ & - & - \\
\hline 77 & $2.08 \pm 0.07$ & $0.19 \pm 0.03$ & - & - \\
\hline 78 & $2.37 \pm 0.06$ & $2.03 \pm 0.33$ & $-0.04 \pm 0.09$ & $-0.82 \pm 0.09$ \\
\hline 79 & $2.20 \pm 0.06$ & $0.44 \pm 0.07$ & - & - \\
\hline 80 & $2.72 \pm 0.09$ & $0.14 \pm 0.03$ & - & - \\
\hline 81 & $2.59 \pm 0.06$ & $0.27 \pm 0.03$ & - & - \\
\hline 82 & $2.10 \pm 0.05$ & $1.30 \pm 0.15$ & $-0.02 \pm 0.08$ & $-0.66 \pm 0.07$ \\
\hline 83 & $2.70 \pm 0.13$ & $0.09 \pm 0.03$ & - & - \\
\hline 84 & $2.34 \pm 0.05$ & $16.85 \pm 2.32$ & $0.15 \pm 0.06$ & $-0.32 \pm 0.06$ \\
\hline 85 & $2.48 \pm 0.04$ & $0.43 \pm 0.04$ & $0.51 \pm 0.06$ & $-0.64 \pm 0.06$ \\
\hline 86 & $2.10 \pm 0.06$ & $4.02 \pm 0.64$ & - & - \\
\hline 87 & $0.98 \pm 0.01$ & $81.20 \pm 2.46$ & $-1.84 \pm 0.03$ & - \\
\hline 88 & $2.48 \pm 0.05$ & $0.43 \pm 0.05$ & $0.40 \pm 0.09$ & $-0.35 \pm 0.08$ \\
\hline 89 & $2.87 \pm 0.07$ & $0.27 \pm 0.04$ & - & - \\
\hline 90 & $1.98 \pm 0.07$ & $0.34 \pm 0.06$ & - & - \\
\hline 91 & $3.26 \pm 0.06$ & $0.35 \pm 0.04$ & - & - \\
\hline 92 & $2.35 \pm 0.05$ & $0.23 \pm 0.02$ & $0.37 \pm 0.09$ & - \\
\hline 93 & $1.99 \pm 0.05$ & $1.75 \pm 0.21$ & $-0.06 \pm 0.08$ & $-0.48 \pm 0.06$ \\
\hline 94 & $2.29 \pm 0.04$ & $1.20 \pm 0.12$ & $0.48 \pm 0.06$ & $-0.13 \pm 0.05$ \\
\hline 95 & $2.54 \pm 0.11$ & $0.14 \pm 0.05$ & - & - \\
\hline 96 & $2.84 \pm 0.06$ & $0.22 \pm 0.03$ & - & - \\
\hline 97 & $1.80 \pm 0.07$ & $0.17 \pm 0.03$ & - & - \\
\hline 98 & $2.69 \pm 0.09$ & $0.20 \pm 0.05$ & - & - \\
\hline 99 & $2.60 \pm 0.04$ & $0.79 \pm 0.06$ & $0.45 \pm 0.05$ & $-0.26 \pm 0.05$ \\
\hline 100 & $2.51 \pm 0.09$ & $0.28 \pm 0.07$ & - & - \\
\hline 101 & $2.12 \pm 0.06$ & $3.23 \pm 0.51$ & - & - \\
\hline 102 & $2.19 \pm 0.05$ & $0.91 \pm 0.12$ & $0.40 \pm 0.08$ & $-0.01 \pm 0.07$ \\
\hline 103 & $2.33 \pm 0.07$ & $0.17 \pm 0.03$ & - & - \\
\hline 104 & $1.30 \pm 0.04$ & $0.74 \pm 0.06$ & $-0.23 \pm 0.07$ & $-1.51 \pm 0.07$ \\
\hline 105 & $2.02 \pm 0.06$ & $0.25 \pm 0.04$ & - & - \\
\hline 106 & $2.26 \pm 0.06$ & $1.48 \pm 0.22$ & $0.18 \pm 0.08$ & $-0.58 \pm 0.08$ \\
\hline 107 & $2.60 \pm 0.05$ & $2.95 \pm 0.39$ & $0.54 \pm 0.06$ & $0.02 \pm 0.06$ \\
\hline 108 & $1.95 \pm 0.06$ & $1.28 \pm 0.20$ & - & - \\
\hline 109 & $2.33 \pm 0.09$ & $0.23 \pm 0.06$ & - & - \\
\hline 110 & $2.17 \pm 0.07$ & $0.24 \pm 0.04$ & - & - \\
\hline 111 & $1.90 \pm 0.06$ & $0.16 \pm 0.02$ & - & - \\
\hline 112 & $1.81 \pm 0.06$ & $0.35 \pm 0.05$ & - & - \\
\hline 113 & $1.76 \pm 0.06$ & $1.49 \pm 0.22$ & - & - \\
\hline 114 & $1.96 \pm 0.07$ & $0.82 \pm 0.16$ & - & - \\
\hline 115 & $2.12 \pm 0.08$ & $0.20 \pm 0.05$ & - & - \\
\hline 116 & $1.61 \pm 0.10$ & $0.16 \pm 0.05$ & - & - \\
\hline 117 & $2.10 \pm 0.06$ & $0.55 \pm 0.08$ & - & - \\
\hline 118 & $1.85 \pm 0.05$ & $0.52 \pm 0.06$ & - & - \\
\hline 119 & $2.41 \pm 0.07$ & $0.66 \pm 0.12$ & - & - \\
\hline 120 & $2.51 \pm 0.06$ & $0.62 \pm 0.10$ & - & - \\
\hline 121 & $3.00 \pm 0.05$ & $0.74 \pm 0.07$ & $0.35 \pm 0.07$ & $-1.03 \pm 0.07$ \\
\hline 122 & $2.30 \pm 0.06$ & $3.44 \pm 0.58$ & $0.11 \pm 0.08$ & $-0.57 \pm 0.08$ \\
\hline 123 & $1.61 \pm 0.05$ & $0.39 \pm 0.04$ & $0.05 \pm 0.08$ & $-0.70 \pm 0.08$ \\
\hline 124 & $2.77 \pm 0.06$ & $0.37 \pm 0.05$ & - & - \\
\hline 125 & $2.07 \pm 0.06$ & $1.98 \pm 0.31$ & $-0.28 \pm 0.11$ & $-0.60 \pm 0.08$ \\
\hline 126 & $1.80 \pm 0.05$ & $3.13 \pm 0.38$ & - & - \\
\hline 127 & $2.18 \pm 0.06$ & $0.23 \pm 0.03$ & - & - \\
\hline 128 & $0.76 \pm 0.05$ & $13.78 \pm 1.75$ & - & - \\
\hline
\end{tabular}


Table 6. continued.

\begin{tabular}{|c|c|c|c|c|}
\hline Number & $\log (\mathrm{EWH} \alpha)(\AA)$ & $F_{\mathrm{H} \beta}\left(10^{14} \mathrm{erg} / \mathrm{cm}^{2} / \mathrm{s}\right)$ & $\log ([\mathrm{OII}] / \mathrm{H} \beta)$ & $\log ([\mathrm{OIII}] / \mathrm{H} \beta)$ \\
\hline 129 & $2.11 \pm 0.06$ & $0.31 \pm 0.04$ & - & - \\
\hline 130 & $1.15 \pm 0.02$ & $0.81 \pm 0.04$ & - & - \\
\hline 131 & $3.12 \pm 0.05$ & $0.96 \pm 0.11$ & $0.10 \pm 0.08$ & $-0.97 \pm 0.08$ \\
\hline 132 & $3.11 \pm 0.07$ & $0.25 \pm 0.03$ & - & - \\
\hline 133 & $2.27 \pm 0.08$ & $0.16 \pm 0.03$ & - & - \\
\hline 134 & $2.67 \pm 0.06$ & $0.66 \pm 0.11$ & - & - \\
\hline 135 & $2.58 \pm 0.08$ & $0.61 \pm 0.15$ & $0.48 \pm 0.13$ & $-0.02 \pm 0.11$ \\
\hline 136 & $2.67 \pm 0.05$ & $0.32 \pm 0.03$ & - & - \\
\hline 137 & $2.29 \pm 0.05$ & $0.26 \pm 0.03$ & - & - \\
\hline 138 & $2.56 \pm 0.08$ & $0.18 \pm 0.03$ & - & - \\
\hline 139 & $2.01 \pm 0.04$ & $2.40 \pm 0.20$ & $-0.25 \pm 0.07$ & $-1.16 \pm 0.06$ \\
\hline 140 & $2.87 \pm 0.05$ & $0.37 \pm 0.03$ & $-0.62 \pm 0.07$ & - \\
\hline 141 & $2.05 \pm 0.06$ & $0.23 \pm 0.03$ & - & - \\
\hline 142 & $2.90 \pm 0.04$ & $0.92 \pm 0.08$ & $-0.14 \pm 0.07$ & $-0.93 \pm 0.07$ \\
\hline 143 & $1.85 \pm 0.04$ & $0.77 \pm 0.08$ & - & - \\
\hline 144 & $2.25 \pm 0.04$ & $0.78 \pm 0.08$ & $0.19 \pm 0.08$ & $-0.55 \pm 0.07$ \\
\hline 145 & $1.95 \pm 0.04$ & $0.79 \pm 0.06$ & $0.32 \pm 0.06$ & $-0.71 \pm 0.06$ \\
\hline 146 & $2.85 \pm 0.05$ & $0.37 \pm 0.04$ & - & - \\
\hline 147 & $2.13 \pm 0.07$ & $0.24 \pm 0.04$ & - & - \\
\hline 148 & $2.43 \pm 0.07$ & $0.44 \pm 0.08$ & - & - \\
\hline 149 & $1.24 \pm 0.05$ & $0.38 \pm 0.04$ & - & - \\
\hline 150 & $2.27 \pm 0.04$ & $0.79 \pm 0.07$ & $0.52 \pm 0.06$ & $-0.22 \pm 0.05$ \\
\hline 151 & $1.90 \pm 0.07$ & $1.84 \pm 0.35$ & - & - \\
\hline 152 & $2.47 \pm 0.06$ & $1.58 \pm 0.24$ & - & - \\
\hline 153 & $2.11 \pm 0.05$ & $0.26 \pm 0.03$ & $0.59 \pm 0.08$ & $-0.28 \pm 0.07$ \\
\hline 154 & $3.35 \pm 0.08$ & $0.20 \pm 0.03$ & - & - \\
\hline 155 & $2.29 \pm 0.05$ & $7.75 \pm 1.07$ & $0.16 \pm 0.06$ & $-0.60 \pm 0.06$ \\
\hline 156 & $2.23 \pm 0.05$ & $2.57 \pm 0.31$ & $-0.13 \pm 0.08$ & $-0.88 \pm 0.07$ \\
\hline 157 & $1.93 \pm 0.05$ & $1.40 \pm 0.16$ & - & - \\
\hline 158 & $2.71 \pm 0.04$ & $0.64 \pm 0.06$ & $-0.02 \pm 0.08$ & $-0.53 \pm 0.07$ \\
\hline 159 & $2.09 \pm 0.06$ & $1.47 \pm 0.23$ & - & - \\
\hline 160 & $3.47 \pm 0.10$ & $0.15 \pm 0.03$ & - & - \\
\hline 161 & $2.07 \pm 0.06$ & $0.30 \pm 0.04$ & - & - \\
\hline 162 & $2.41 \pm 0.05$ & $3.93 \pm 0.52$ & $-0.05 \pm 0.07$ & $-0.73 \pm 0.07$ \\
\hline 163 & $2.21 \pm 0.06$ & $0.23 \pm 0.03$ & - & - \\
\hline 164 & $2.41 \pm 0.05$ & $0.43 \pm 0.05$ & - & - \\
\hline 165 & $2.32 \pm 0.05$ & $0.26 \pm 0.03$ & - & - \\
\hline 166 & $0.58 \pm 0.07$ & $1.15 \pm 0.20$ & - & - \\
\hline 167 & $2.02 \pm 0.04$ & $1.24 \pm 0.10$ & $0.14 \pm 0.06$ & $-0.25 \pm 0.04$ \\
\hline 168 & $2.50 \pm 0.06$ & $0.97 \pm 0.16$ & $0.11 \pm 0.11$ & $-0.80 \pm 0.11$ \\
\hline 169 & $2.61 \pm 0.06$ & $1.23 \pm 0.17$ & $0.06 \pm 0.09$ & $-0.81 \pm 0.09$ \\
\hline 170 & $1.94 \pm 0.06$ & $0.74 \pm 0.11$ & - & - \\
\hline 171 & $2.25 \pm 0.05$ & $0.29 \pm 0.03$ & $0.51 \pm 0.09$ & $-0.92 \pm 0.08$ \\
\hline 172 & $2.41 \pm 0.05$ & $0.94 \pm 0.10$ & - & - \\
\hline 173 & $2.02 \pm 0.06$ & $0.27 \pm 0.03$ & - & - \\
\hline 174 & $2.64 \pm 0.06$ & $0.68 \pm 0.09$ & - & - \\
\hline 175 & $2.19 \pm 0.03$ & $1.25 \pm 0.08$ & $0.39 \pm 0.04$ & $-0.71 \pm 0.04$ \\
\hline 176 & $2.00 \pm 0.05$ & $1.73 \pm 0.19$ & - & - \\
\hline 177 & $4.57 \pm 0.10$ & $0.38 \pm 0.03$ & - & - \\
\hline 178 & $2.78 \pm 0.07$ & $0.18 \pm 0.03$ & - & - \\
\hline 179 & $2.23 \pm 0.05$ & $0.50 \pm 0.06$ & - & - \\
\hline 180 & $2.42 \pm 0.04$ & $7.35 \pm 0.71$ & $0.59 \pm 0.04$ & $-0.12 \pm 0.04$ \\
\hline 181 & $2.42 \pm 0.05$ & $0.32 \pm 0.03$ & $0.41 \pm 0.07$ & - \\
\hline 182 & $2.33 \pm 0.05$ & $2.24 \pm 0.25$ & $0.28 \pm 0.06$ & $-0.54 \pm 0.06$ \\
\hline 183 & $2.54 \pm 0.02$ & $1.08 \pm 0.05$ & - & - \\
\hline 184 & $2.05 \pm 0.05$ & $0.65 \pm 0.08$ & $0.38 \pm 0.08$ & $-0.45 \pm 0.08$ \\
\hline 185 & $2.92 \pm 0.06$ & $0.87 \pm 0.12$ & - & - \\
\hline
\end{tabular}


B. Cedrés et al.: Two-dimensional metallicity distribution in NGC 628 and NGC 6946

Table 6. continued.

\begin{tabular}{lcccc}
\hline \hline Number & $\log (\mathrm{EWH} \alpha)(\AA)$ & $F_{\mathrm{H} \beta}\left(10^{14} \mathrm{erg} / \mathrm{cm}^{2} / \mathrm{s}\right)$ & $\log ([\mathrm{OII}] / \mathrm{H} \beta)$ & $\log ([\mathrm{OIII}] / \mathrm{H} \beta)$ \\
\hline 186 & $1.19 \pm 0.02$ & $0.87 \pm 0.05$ & $0.33 \pm 0.04$ & - \\
187 & $2.04 \pm 0.04$ & $9.65 \pm 0.99$ & $-0.29 \pm 0.05$ & $-0.42 \pm 0.05$ \\
188 & $2.28 \pm 0.04$ & $2.68 \pm 0.29$ & $0.21 \pm 0.06$ & $-0.54 \pm 0.05$ \\
189 & $2.95 \pm 0.05$ & $0.28 \pm 0.02$ & - & - \\
190 & $2.10 \pm 0.06$ & $0.81 \pm 0.12$ & $0.16 \pm 0.10$ & $-0.55 \pm 0.09$ \\
191 & $2.20 \pm 0.04$ & $0.45 \pm 0.03$ & $0.13 \pm 0.05$ & $-0.74 \pm 0.06$ \\
192 & $2.32 \pm 0.05$ & $1.00 \pm 0.12$ & $0.33 \pm 0.07$ & $-0.88 \pm 0.09$ \\
193 & $2.51 \pm 0.05$ & $4.05 \pm 0.47$ & $0.21 \pm 0.06$ & $-0.58 \pm 0.06$ \\
194 & $2.13 \pm 0.05$ & $0.25 \pm 0.03$ & $0.25 \pm 0.08$ & $-0.41 \pm 0.08$ \\
195 & $2.29 \pm 0.04$ & $0.35 \pm 0.03$ & $0.32 \pm 0.07$ & $-0.25 \pm 0.07$ \\
196 & $2.33 \pm 0.04$ & $3.42 \pm 0.29$ & $0.41 \pm 0.04$ & $-0.18 \pm 0.04$ \\
197 & $2.30 \pm 0.06$ & $0.38 \pm 0.05$ & $0.48 \pm 0.10$ & $-0.29 \pm 0.09$ \\
198 & $2.05 \pm 0.04$ & $1.06 \pm 0.09$ & $0.65 \pm 0.05$ & $-0.03 \pm 0.04$ \\
199 & $2.76 \pm 0.04$ & $0.38 \pm 0.03$ & - & - \\
200 & $2.17 \pm 0.03$ & $7.05 \pm 0.57$ & $0.19 \pm 0.04$ & $-0.37 \pm 0.04$ \\
201 & $2.27 \pm 0.04$ & $0.79 \pm 0.06$ & $0.01 \pm 0.07$ & $-0.93 \pm 0.06$ \\
202 & $2.34 \pm 0.05$ & $12.54 \pm 1.64$ & $0.44 \pm 0.06$ & $-0.35 \pm 0.06$ \\
203 & $2.30 \pm 0.05$ & $2.03 \pm 0.24$ & $0.39 \pm 0.06$ & $-0.57 \pm 0.06$ \\
204 & $2.35 \pm 0.06$ & $1.01 \pm 0.14$ & $0.51 \pm 0.07$ & $0.15 \pm 0.06$ \\
205 & $2.12 \pm 0.04$ & $23.17 \pm 2.35$ & $0.46 \pm 0.04$ & $-0.09 \pm 0.04$ \\
206 & $3.06 \pm 0.03$ & $1.57 \pm 0.11$ & $0.53 \pm 0.04$ & $-0.25 \pm 0.04$ \\
207 & $1.88 \pm 0.03$ & $2.35 \pm 0.18$ & $0.42 \pm 0.04$ & $-0.15 \pm 0.04$ \\
208 & $2.57 \pm 0.05$ & $0.40 \pm 0.04$ & $0.29 \pm 0.07$ & $-0.30 \pm 0.07$ \\
209 & $1.98 \pm 0.02$ & $0.95 \pm 0.05$ & - & - \\
\hline
\end{tabular}


Table 7. Equivalent width, $\mathrm{H} \beta$ flux and oxygen flux ratios, both extinction-corrected, for all the H II regions of NGC 6946.

\begin{tabular}{|c|c|c|c|c|}
\hline Number & $\log (\mathrm{EWH} \alpha)(\AA)$ & $F_{\mathrm{H} \beta}\left(10^{14} \mathrm{erg} / \mathrm{cm}^{2} / \mathrm{s}\right)$ & $\log ([\mathrm{OII}] / \mathrm{H} \beta)$ & $\log ([\mathrm{OIII}] / \mathrm{H} \beta)$ \\
\hline 1 & $2.97 \pm 0.13$ & $0.20 \pm 0.06$ & - & - \\
\hline 2 & $2.86 \pm 0.10$ & $0.21 \pm 0.04$ & $0.53 \pm 0.09$ & $-0.43 \pm 0.09$ \\
\hline 3 & $2.69 \pm 0.05$ & $24.05 \pm 2.80$ & $0.45 \pm 0.05$ & $-0.09 \pm 0.05$ \\
\hline 4 & $2.59 \pm 0.08$ & $0.23 \pm 0.03$ & - & - \\
\hline 5 & $3.25 \pm 0.08$ & $0.25 \pm 0.03$ & $0.53 \pm 0.06$ & $-0.48 \pm 0.06$ \\
\hline 6 & $3.11 \pm 0.05$ & $0.90 \pm 0.08$ & $0.49 \pm 0.04$ & $-0.01 \pm 0.04$ \\
\hline 7 & $2.88 \pm 0.06$ & $1.17 \pm 0.13$ & $0.38 \pm 0.05$ & $-0.56 \pm 0.05$ \\
\hline 8 & $2.54 \pm 0.08$ & $1.04 \pm 0.20$ & $0.34 \pm 0.08$ & $-0.52 \pm 0.08$ \\
\hline 9 & $2.92 \pm 0.07$ & $0.44 \pm 0.05$ & $0.50 \pm 0.05$ & $-0.61 \pm 0.05$ \\
\hline 10 & $2.89 \pm 0.05$ & $2.11 \pm 0.20$ & - & - \\
\hline 11 & $3.04 \pm 0.09$ & $0.62 \pm 0.12$ & $0.48 \pm 0.09$ & $0.05 \pm 0.09$ \\
\hline 12 & $2.85 \pm 0.11$ & $0.22 \pm 0.05$ & $0.51 \pm 0.11$ & $0.41 \pm 0.11$ \\
\hline 13 & $3.55 \pm 0.10$ & $0.16 \pm 0.02$ & $0.43 \pm 0.06$ & - \\
\hline 14 & $2.83 \pm 0.05$ & $2.94 \pm 0.30$ & $0.28 \pm 0.04$ & $-0.37 \pm 0.05$ \\
\hline 15 & $3.48 \pm 0.09$ & $0.22 \pm 0.03$ & $0.36 \pm 0.07$ & $-0.21 \pm 0.07$ \\
\hline 16 & $3.12 \pm 0.10$ & $0.18 \pm 0.03$ & $0.68 \pm 0.13$ & $0.04 \pm 0.11$ \\
\hline 17 & $2.68 \pm 0.11$ & $0.11 \pm 0.02$ & - & - \\
\hline 18 & $2.55 \pm 0.07$ & $10.05 \pm 1.74$ & $0.29 \pm 0.07$ & $-0.06 \pm 0.07$ \\
\hline 19 & $2.64 \pm 0.12$ & $0.13 \pm 0.03$ & - & - \\
\hline 20 & $2.37 \pm 0.09$ & $0.20 \pm 0.04$ & - & - \\
\hline 21 & $2.71 \pm 0.06$ & $2.16 \pm 0.29$ & $0.38 \pm 0.06$ & $-0.29 \pm 0.06$ \\
\hline 22 & $2.97 \pm 0.07$ & $1.89 \pm 0.26$ & $0.49 \pm 0.06$ & $-0.24 \pm 0.06$ \\
\hline 23 & $1.83 \pm 0.08$ & $0.56 \pm 0.09$ & - & - \\
\hline 24 & $2.35 \pm 0.07$ & $0.81 \pm 0.13$ & $0.57 \pm 0.07$ & $-0.19 \pm 0.07$ \\
\hline 25 & $2.46 \pm 0.09$ & $0.63 \pm 0.14$ & - & - \\
\hline 26 & $2.98 \pm 0.05$ & $1.55 \pm 0.16$ & $0.39 \pm 0.04$ & $-0.44 \pm 0.04$ \\
\hline 27 & $2.84 \pm 0.07$ & $0.81 \pm 0.12$ & $0.23 \pm 0.06$ & $-0.40 \pm 0.08$ \\
\hline 28 & $2.47 \pm 0.06$ & $0.92 \pm 0.10$ & $0.18 \pm 0.08$ & $-0.38 \pm 0.05$ \\
\hline 29 & $2.69 \pm 0.12$ & $0.22 \pm 0.07$ & - & - \\
\hline 30 & $2.62 \pm 0.10$ & $0.19 \pm 0.04$ & - & - \\
\hline 31 & $2.67 \pm 0.06$ & $1.64 \pm 0.22$ & - & - \\
\hline 32 & $2.27 \pm 0.11$ & $0.41 \pm 0.11$ & - & - \\
\hline 33 & $2.77 \pm 0.10$ & $0.29 \pm 0.07$ & $0.19 \pm 0.10$ & $-0.58 \pm 0.16$ \\
\hline 34 & $2.62 \pm 0.04$ & $34.62 \pm 3.33$ & $0.11 \pm 0.04$ & $-0.54 \pm 0.04$ \\
\hline 35 & $3.74 \pm 0.12$ & $0.17 \pm 0.03$ & - & - \\
\hline 36 & $3.06 \pm 0.06$ & $0.70 \pm 0.07$ & $0.43 \pm 0.04$ & $-0.67 \pm 0.04$ \\
\hline 37 & $2.58 \pm 0.09$ & $5.32 \pm 1.20$ & - & - \\
\hline 38 & $2.76 \pm 0.06$ & $5.32 \pm 0.73$ & $0.22 \pm 0.06$ & $-0.46 \pm 0.06$ \\
\hline 39 & $2.71 \pm 0.07$ & $0.79 \pm 0.11$ & - & - \\
\hline 40 & $2.55 \pm 0.06$ & $1.48 \pm 0.20$ & - & - \\
\hline 41 & $2.64 \pm 0.07$ & $1.15 \pm 0.19$ & - & - \\
\hline 42 & $2.27 \pm 0.09$ & $3.55 \pm 0.80$ & - & - \\
\hline 43 & $2.60 \pm 0.06$ & $3.50 \pm 0.48$ & - & - \\
\hline 44 & $2.75 \pm 0.06$ & $0.36 \pm 0.03$ & $0.16 \pm 0.04$ & $-0.73 \pm 0.04$ \\
\hline 45 & $2.51 \pm 0.07$ & $1.31 \pm 0.19$ & - & - \\
\hline 46 & $2.41 \pm 0.11$ & $0.13 \pm 0.03$ & - & - \\
\hline 47 & $3.15 \pm 0.14$ & $0.18 \pm 0.06$ & - & - \\
\hline 48 & $2.75 \pm 0.04$ & $5.56 \pm 0.51$ & $0.08 \pm 0.04$ & $-0.66 \pm 0.04$ \\
\hline 49 & $2.63 \pm 0.10$ & $0.13 \pm 0.03$ & - & - \\
\hline 50 & $2.56 \pm 0.08$ & $0.44 \pm 0.08$ & - & - \\
\hline 51 & $2.66 \pm 0.09$ & $0.36 \pm 0.07$ & - & - \\
\hline 52 & $2.31 \pm 0.09$ & $2.88 \pm 0.67$ & - & - \\
\hline 53 & $2.81 \pm 0.05$ & $2.02 \pm 0.20$ & $0.27 \pm 0.04$ & $-0.38 \pm 0.04$ \\
\hline 54 & $2.17 \pm 0.09$ & $1.66 \pm 0.40$ & - & - \\
\hline 55 & $2.71 \pm 0.04$ & $7.05 \pm 0.53$ & $0.09 \pm 0.04$ & $-0.73 \pm 0.03$ \\
\hline 56 & $2.62 \pm 0.10$ & $0.34 \pm 0.08$ & - & - \\
\hline 57 & $3.52 \pm 0.07$ & $0.35 \pm 0.04$ & $0.27 \pm 0.05$ & $-0.09 \pm 0.05$ \\
\hline 58 & $3.39 \pm 0.08$ & $0.25 \pm 0.03$ & $0.22 \pm 0.06$ & $-0.72 \pm 0.06$ \\
\hline 59 & $2.28 \pm 0.11$ & $0.51 \pm 0.14$ & - & - \\
\hline 60 & $2.75 \pm 0.07$ & $2.63 \pm 0.43$ & - & - \\
\hline 61 & $2.49 \pm 0.05$ & $2.75 \pm 0.29$ & - & - \\
\hline 62 & $3.17 \pm 0.13$ & $0.10 \pm 0.03$ & - & - \\
\hline 63 & $2.90 \pm 0.03$ & $13.25 \pm 0.82$ & $-0.00 \pm 0.03$ & $-0.61 \pm 0.03$ \\
\hline 64 & $2.97 \pm 0.05$ & $1.58 \pm 0.15$ & $0.19 \pm 0.06$ & $-0.62 \pm 0.05$ \\
\hline 65 & $2.50 \pm 0.10$ & $0.43 \pm 0.10$ & $0.23 \pm 0.16$ & $-0.37 \pm 0.13$ \\
\hline 66 & $2.49 \pm 0.08$ & $3.02 \pm 0.62$ & $-0.08 \pm 0.11$ & $-0.46 \pm 0.09$ \\
\hline
\end{tabular}


Table 7. continued.

\begin{tabular}{|c|c|c|c|c|}
\hline Number & $\log (\mathrm{EWH} \alpha)(\AA)$ & $F_{\mathrm{H} \beta}\left(10^{14} \mathrm{erg} / \mathrm{cm}^{2} / \mathrm{s}\right)$ & $\log ([\mathrm{OII}] / \mathrm{H} \beta)$ & $\log ([\mathrm{OIII}] / \mathrm{H} \beta)$ \\
\hline 67 & $2.97 \pm 0.07$ & $0.29 \pm 0.03$ & - & - \\
\hline 68 & $2.99 \pm 0.11$ & $0.34 \pm 0.08$ & - & - \\
\hline 69 & $2.24 \pm 0.10$ & $0.13 \pm 0.03$ & - & - \\
\hline 70 & $2.72 \pm 0.07$ & $0.45 \pm 0.07$ & - & - \\
\hline 71 & $2.48 \pm 0.09$ & $0.42 \pm 0.08$ & - & - \\
\hline 72 & $2.69 \pm 0.05$ & $0.70 \pm 0.06$ & $0.59 \pm 0.05$ & $-0.30 \pm 0.05$ \\
\hline 73 & $2.54 \pm 0.11$ & $0.22 \pm 0.05$ & - & - \\
\hline 74 & $2.68 \pm 0.12$ & $0.28 \pm 0.08$ & - & - \\
\hline 75 & $2.32 \pm 0.09$ & $1.10 \pm 0.23$ & - & - \\
\hline 76 & $2.50 \pm 0.14$ & $0.12 \pm 0.04$ & - & - \\
\hline 77 & $2.76 \pm 0.09$ & $0.64 \pm 0.14$ & - & - \\
\hline 78 & $2.88 \pm 0.07$ & $1.85 \pm 0.30$ & $0.04 \pm 0.07$ & $-0.57 \pm 0.07$ \\
\hline 79 & $2.25 \pm 0.11$ & $0.16 \pm 0.04$ & - & - \\
\hline 80 & $2.70 \pm 0.05$ & $2.25 \pm 0.23$ & $0.02 \pm 0.04$ & $-0.72 \pm 0.05$ \\
\hline 81 & $2.68 \pm 0.07$ & $0.32 \pm 0.04$ & - & - \\
\hline 82 & $2.52 \pm 0.14$ & $0.13 \pm 0.05$ & - & - \\
\hline 83 & $2.43 \pm 0.09$ & $0.82 \pm 0.17$ & - & - \\
\hline 84 & $2.78 \pm 0.09$ & $1.28 \pm 0.26$ & - & - \\
\hline 85 & $2.37 \pm 0.05$ & $1.59 \pm 0.14$ & $0.02 \pm 0.06$ & $-0.23 \pm 0.04$ \\
\hline 86 & $2.52 \pm 0.10$ & $0.23 \pm 0.05$ & - & - \\
\hline 87 & $2.84 \pm 0.13$ & $0.13 \pm 0.04$ & - & - \\
\hline 88 & $2.69 \pm 0.12$ & $0.15 \pm 0.04$ & - & - \\
\hline 89 & $2.36 \pm 0.09$ & $0.24 \pm 0.05$ & - & - \\
\hline 90 & $2.23 \pm 0.07$ & $0.56 \pm 0.08$ & - & - \\
\hline 91 & $2.54 \pm 0.08$ & $0.62 \pm 0.10$ & - & - \\
\hline 92 & $2.01 \pm 0.10$ & $0.63 \pm 0.16$ & - & - \\
\hline 93 & $2.52 \pm 0.10$ & $0.29 \pm 0.06$ & - & - \\
\hline 94 & $2.68 \pm 0.11$ & $0.64 \pm 0.17$ & - & - \\
\hline 95 & $2.63 \pm 0.05$ & $2.11 \pm 0.22$ & - & - \\
\hline 96 & $2.32 \pm 0.06$ & $2.02 \pm 0.25$ & - & - \\
\hline 97 & $2.50 \pm 0.12$ & $0.16 \pm 0.04$ & - & - \\
\hline 98 & $2.73 \pm 0.06$ & $6.43 \pm 0.88$ & - & - \\
\hline 99 & $2.34 \pm 0.10$ & $0.68 \pm 0.17$ & - & - \\
\hline 100 & $2.47 \pm 0.08$ & $0.43 \pm 0.07$ & - & - \\
\hline 101 & $3.04 \pm 0.09$ & $0.94 \pm 0.18$ & - & - \\
\hline 102 & $3.52 \pm 0.18$ & $0.06 \pm 0.02$ & - & - \\
\hline 103 & $2.92 \pm 0.06$ & $0.85 \pm 0.09$ & - & - \\
\hline 104 & $2.84 \pm 0.08$ & $0.27 \pm 0.04$ & - & - \\
\hline 105 & $2.37 \pm 0.08$ & $2.77 \pm 0.55$ & - & - \\
\hline 106 & $2.39 \pm 0.16$ & $0.08 \pm 0.03$ & - & - \\
\hline 107 & $2.48 \pm 0.09$ & $0.91 \pm 0.19$ & - & - \\
\hline 108 & $2.62 \pm 0.07$ & $0.99 \pm 0.14$ & - & - \\
\hline 109 & $2.74 \pm 0.06$ & $1.17 \pm 0.13$ & - & - \\
\hline 110 & $2.42 \pm 0.07$ & $0.43 \pm 0.06$ & - & - \\
\hline 111 & $2.66 \pm 0.07$ & $0.30 \pm 0.03$ & $0.61 \pm 0.09$ & - \\
\hline 112 & $2.48 \pm 0.05$ & $0.67 \pm 0.06$ & - & - \\
\hline 113 & $2.38 \pm 0.05$ & $4.82 \pm 0.51$ & $0.03 \pm 0.05$ & $-0.87 \pm 0.05$ \\
\hline 114 & $2.07 \pm 0.11$ & $0.21 \pm 0.06$ & - & - \\
\hline 115 & $3.54 \pm 0.05$ & $2.30 \pm 0.21$ & - & - \\
\hline 116 & $2.64 \pm 0.10$ & $0.34 \pm 0.08$ & - & - \\
\hline 117 & $2.47 \pm 0.05$ & $1.25 \pm 0.11$ & $0.22 \pm 0.04$ & $-0.58 \pm 0.05$ \\
\hline 118 & $1.54 \pm 0.07$ & $19.32 \pm 3.13$ & - & - \\
\hline 119 & $2.45 \pm 0.08$ & $0.35 \pm 0.05$ & - & - \\
\hline 120 & $2.73 \pm 0.08$ & $0.38 \pm 0.06$ & - & - \\
\hline 121 & $2.59 \pm 0.04$ & $0.77 \pm 0.05$ & $-0.06 \pm 0.10$ & $-0.63 \pm 0.03$ \\
\hline 122 & $2.52 \pm 0.11$ & $0.12 \pm 0.03$ & - & - \\
\hline 123 & $2.38 \pm 0.07$ & $2.59 \pm 0.45$ & - & - \\
\hline 124 & $2.33 \pm 0.09$ & $0.67 \pm 0.14$ & - & - \\
\hline 125 & $2.33 \pm 0.06$ & $0.67 \pm 0.08$ & - & - \\
\hline 126 & $3.11 \pm 0.05$ & $0.44 \pm 0.03$ & - & - \\
\hline 127 & $2.72 \pm 0.09$ & $1.34 \pm 0.32$ & - & - \\
\hline 128 & $2.08 \pm 0.08$ & $0.33 \pm 0.06$ & - & - \\
\hline 129 & $2.78 \pm 0.04$ & $0.79 \pm 0.05$ & $0.09 \pm 0.07$ & $-0.65 \pm 0.03$ \\
\hline 130 & $2.35 \pm 0.04$ & $4.67 \pm 0.35$ & $0.12 \pm 0.03$ & $-0.53 \pm 0.03$ \\
\hline 131 & $2.26 \pm 0.10$ & $0.29 \pm 0.07$ & - & - \\
\hline 132 & $1.78 \pm 0.07$ & $1.20 \pm 0.19$ & - & - \\
\hline
\end{tabular}


Table 7. continued.

\begin{tabular}{|c|c|c|c|c|}
\hline Number & $\log (\mathrm{EWH} \alpha)(\AA)$ & $F_{\mathrm{H} \beta}\left(10^{14} \mathrm{erg} / \mathrm{cm}^{2} / \mathrm{s}\right)$ & $\log ([\mathrm{OII}] / \mathrm{H} \beta)$ & $\log ([\mathrm{OIII}] / \mathrm{H} \beta)$ \\
\hline 133 & $2.36 \pm 0.08$ & $3.49 \pm 0.73$ & - & - \\
\hline 134 & $2.32 \pm 0.06$ & $0.53 \pm 0.05$ & $0.18 \pm 0.04$ & $-0.72 \pm 0.05$ \\
\hline 135 & $2.49 \pm 0.03$ & $59.30 \pm 3.86$ & $0.33 \pm 0.03$ & $-0.59 \pm 0.03$ \\
\hline 136 & $2.43 \pm 0.05$ & $0.62 \pm 0.05$ & $0.41 \pm 0.07$ & $-0.47 \pm 0.04$ \\
\hline 137 & $2.44 \pm 0.13$ & $0.16 \pm 0.05$ & - & - \\
\hline 138 & $2.78 \pm 0.06$ & $0.27 \pm 0.03$ & $0.11 \pm 0.04$ & $-0.52 \pm 0.04$ \\
\hline 139 & $2.44 \pm 0.07$ & $1.05 \pm 0.16$ & - & - \\
\hline 140 & $2.58 \pm 0.09$ & $0.37 \pm 0.08$ & - & - \\
\hline 141 & $3.05 \pm 0.09$ & $0.21 \pm 0.03$ & - & - \\
\hline 142 & $2.30 \pm 0.05$ & $1.17 \pm 0.12$ & $-0.05 \pm 0.04$ & $-0.90 \pm 0.08$ \\
\hline 143 & $2.46 \pm 0.04$ & $1.04 \pm 0.07$ & - & - \\
\hline 144 & $2.54 \pm 0.02$ & $18.47 \pm 0.65$ & - & - \\
\hline 145 & $2.34 \pm 0.07$ & $2.93 \pm 0.44$ & - & - \\
\hline 146 & $2.65 \pm 0.05$ & $3.36 \pm 0.34$ & $-0.08 \pm 0.04$ & $-0.78 \pm 0.04$ \\
\hline 147 & $2.40 \pm 0.04$ & $2.29 \pm 0.16$ & - & - \\
\hline 148 & $3.14 \pm 0.06$ & $0.37 \pm 0.03$ & $0.23 \pm 0.11$ & $-0.36 \pm 0.04$ \\
\hline 149 & $2.31 \pm 0.06$ & $0.64 \pm 0.07$ & - & - \\
\hline 150 & $2.40 \pm 0.08$ & $1.33 \pm 0.23$ & - & - \\
\hline 151 & $2.76 \pm 0.06$ & $0.47 \pm 0.05$ & $0.20 \pm 0.11$ & $-0.47 \pm 0.08$ \\
\hline 152 & $2.83 \pm 0.06$ & $0.70 \pm 0.08$ & $0.39 \pm 0.05$ & $0.05 \pm 0.05$ \\
\hline 153 & $2.07 \pm 0.04$ & $2.06 \pm 0.13$ & $0.68 \pm 0.03$ & $-0.24 \pm 0.03$ \\
\hline 154 & $2.62 \pm 0.03$ & $4.11 \pm 0.25$ & $0.22 \pm 0.03$ & $-0.48 \pm 0.03$ \\
\hline 155 & $2.35 \pm 0.09$ & $0.68 \pm 0.15$ & - & - \\
\hline 156 & $2.70 \pm 0.07$ & $0.94 \pm 0.12$ & - & - \\
\hline 157 & $3.31 \pm 0.07$ & $0.31 \pm 0.03$ & $0.40 \pm 0.05$ & $-0.38 \pm 0.05$ \\
\hline 158 & $2.58 \pm 0.06$ & $0.84 \pm 0.09$ & - & - \\
\hline 159 & $2.71 \pm 0.03$ & $6.33 \pm 0.39$ & $0.17 \pm 0.03$ & $-0.59 \pm 0.03$ \\
\hline 160 & $2.65 \pm 0.09$ & $0.46 \pm 0.09$ & - & - \\
\hline 161 & $2.36 \pm 0.09$ & $0.17 \pm 0.03$ & - & - \\
\hline 162 & $2.36 \pm 0.04$ & $0.61 \pm 0.04$ & - & - \\
\hline 163 & $2.40 \pm 0.04$ & $3.01 \pm 0.23$ & - & - \\
\hline 164 & $2.58 \pm 0.03$ & $1.32 \pm 0.07$ & - & - \\
\hline 165 & $2.33 \pm 0.09$ & $0.20 \pm 0.04$ & - & - \\
\hline 166 & $2.49 \pm 0.05$ & $1.08 \pm 0.12$ & - & - \\
\hline 167 & $2.67 \pm 0.04$ & $2.16 \pm 0.18$ & - & - \\
\hline 168 & $2.80 \pm 0.05$ & $0.98 \pm 0.08$ & $0.19 \pm 0.04$ & $-0.69 \pm 0.04$ \\
\hline 169 & $2.39 \pm 0.07$ & $2.60 \pm 0.38$ & - & - \\
\hline 170 & $2.37 \pm 0.06$ & $0.29 \pm 0.03$ & $0.16 \pm 0.05$ & - \\
\hline 171 & $2.57 \pm 0.04$ & $7.57 \pm 0.69$ & - & - \\
\hline 172 & $2.44 \pm 0.08$ & $0.71 \pm 0.12$ & - & - \\
\hline 173 & $2.75 \pm 0.07$ & $0.27 \pm 0.04$ & $0.38 \pm 0.06$ & $-0.49 \pm 0.06$ \\
\hline 174 & $1.86 \pm 0.11$ & $0.44 \pm 0.12$ & - & - \\
\hline 175 & $2.60 \pm 0.06$ & $1.24 \pm 0.16$ & - & - \\
\hline 176 & $2.93 \pm 0.05$ & $0.86 \pm 0.08$ & $0.17 \pm 0.04$ & $-0.38 \pm 0.04$ \\
\hline 177 & $2.72 \pm 0.05$ & $2.91 \pm 0.31$ & $0.06 \pm 0.05$ & $-0.36 \pm 0.05$ \\
\hline 178 & $2.53 \pm 0.07$ & $0.57 \pm 0.08$ & - & - \\
\hline 179 & $2.58 \pm 0.06$ & $1.13 \pm 0.15$ & - & - \\
\hline 180 & $2.39 \pm 0.05$ & $2.00 \pm 0.18$ & $0.06 \pm 0.04$ & $-0.62 \pm 0.04$ \\
\hline 181 & $2.50 \pm 0.06$ & $0.65 \pm 0.07$ & - & - \\
\hline 182 & $2.60 \pm 0.06$ & $0.59 \pm 0.07$ & - & - \\
\hline 183 & $2.18 \pm 0.09$ & $0.36 \pm 0.07$ & - & - \\
\hline 184 & $2.66 \pm 0.06$ & $0.66 \pm 0.07$ & - & - \\
\hline 185 & $2.44 \pm 0.06$ & $2.87 \pm 0.38$ & - & - \\
\hline 186 & $2.46 \pm 0.07$ & $2.14 \pm 0.32$ & - & - \\
\hline 187 & $2.66 \pm 0.08$ & $0.42 \pm 0.06$ & $0.13 \pm 0.07$ & $-0.42 \pm 0.07$ \\
\hline 188 & $2.28 \pm 0.06$ & $0.22 \pm 0.02$ & $0.17 \pm 0.05$ & $0.11 \pm 0.05$ \\
\hline 189 & $2.92 \pm 0.06$ & $2.96 \pm 0.42$ & - & - \\
\hline 190 & $3.10 \pm 0.06$ & $0.71 \pm 0.08$ & $0.13 \pm 0.05$ & $-0.54 \pm 0.05$ \\
\hline 191 & $2.51 \pm 0.07$ & $4.36 \pm 0.67$ & - & - \\
\hline 192 & $2.70 \pm 0.04$ & $1.03 \pm 0.08$ & - & - \\
\hline 193 & $2.01 \pm 0.09$ & $1.72 \pm 0.42$ & - & - \\
\hline 194 & $2.67 \pm 0.08$ & $0.73 \pm 0.13$ & - & - \\
\hline 195 & $2.34 \pm 0.05$ & $0.38 \pm 0.03$ & $0.61 \pm 0.04$ & $-0.55 \pm 0.04$ \\
\hline 196 & $2.31 \pm 0.03$ & $0.98 \pm 0.06$ & - & - \\
\hline 197 & $2.65 \pm 0.06$ & $4.42 \pm 0.57$ & - & - \\
\hline
\end{tabular}


B. Cedrés et al.: Two-dimensional metallicity distribution in NGC 628 and NGC 6946

Table 7. continued.

\begin{tabular}{lcccc}
\hline \hline Number & $\log (\mathrm{EWH} \alpha)(\AA)$ & $F_{\mathrm{H} \beta}\left(10^{14} \mathrm{erg} / \mathrm{cm}^{2} / \mathrm{s}\right)$ & $\log ([\mathrm{OII}] / \mathrm{H} \beta)$ & $\log ([\mathrm{OIII}] / \mathrm{H} \beta)$ \\
\hline 198 & $2.46 \pm 0.05$ & $1.77 \pm 0.19$ & - & - \\
199 & $2.48 \pm 0.06$ & $0.29 \pm 0.03$ & $0.18 \pm 0.04$ & $-0.35 \pm 0.04$ \\
200 & $2.61 \pm 0.08$ & $0.23 \pm 0.04$ & $0.32 \pm 0.07$ & $-0.10 \pm 0.09$ \\
201 & $2.47 \pm 0.05$ & $6.06 \pm 0.65$ & $-0.08 \pm 0.06$ & $-0.51 \pm 0.05$ \\
202 & $2.76 \pm 0.09$ & $0.25 \pm 0.04$ & - & - \\
203 & $2.66 \pm 0.09$ & $0.17 \pm 0.03$ & - & - \\
204 & $2.64 \pm 0.04$ & $0.61 \pm 0.04$ & - & - \\
205 & $2.59 \pm 0.04$ & $5.44 \pm 0.47$ & $0.13 \pm 0.04$ & $-0.45 \pm 0.04$ \\
206 & $2.61 \pm 0.06$ & $0.93 \pm 0.12$ & $0.20 \pm 0.05$ & $-0.57 \pm 0.05$ \\
207 & $2.25 \pm 0.04$ & $5.47 \pm 0.39$ & $0.65 \pm 0.03$ & $-0.35 \pm 0.03$ \\
208 & $2.58 \pm 0.07$ & $0.31 \pm 0.04$ & - & - \\
209 & $2.26 \pm 0.07$ & $0.81 \pm 0.13$ & - & - \\
210 & $2.73 \pm 0.03$ & $3.88 \pm 0.21$ & $0.37 \pm 0.02$ & $-0.46 \pm 0.02$ \\
211 & $2.77 \pm 0.03$ & $2.98 \pm 0.18$ & $0.36 \pm 0.03$ & $-0.43 \pm 0.03$ \\
212 & $2.60 \pm 0.04$ & $0.40 \pm 0.03$ & - & - \\
213 & $2.50 \pm 0.05$ & $1.53 \pm 0.14$ & $0.26 \pm 0.04$ & $-0.56 \pm 0.04$ \\
214 & $2.49 \pm 0.04$ & $14.40 \pm 1.04$ & $0.33 \pm 0.03$ & $-0.27 \pm 0.03$ \\
215 & $2.33 \pm 0.11$ & $0.25 \pm 0.07$ & - & - \\
216 & $2.47 \pm 0.06$ & $0.55 \pm 0.06$ & $0.40 \pm 0.05$ & $-0.44 \pm 0.05$ \\
217 & $2.87 \pm 0.06$ & $0.29 \pm 0.03$ & $0.62 \pm 0.04$ & - \\
218 & $2.58 \pm 0.06$ & $0.74 \pm 0.09$ & $0.21 \pm 0.05$ & $-0.55 \pm 0.07$ \\
219 & $2.74 \pm 0.06$ & $0.26 \pm 0.02$ & $0.85 \pm 0.04$ & - \\
220 & $2.76 \pm 0.04$ & $0.62 \pm 0.04$ & - & - \\
221 & $2.41 \pm 0.04$ & $0.75 \pm 0.06$ & $0.37 \pm 0.03$ & $-0.59 \pm 0.06$ \\
222 & $2.44 \pm 0.03$ & $0.97 \pm 0.05$ & - & - \\
223 & $2.59 \pm 0.03$ & $1.05 \pm 0.05$ & $0.41 \pm 0.02$ & $-0.28 \pm 0.02$ \\
224 & $2.14 \pm 0.05$ & $0.78 \pm 0.08$ & $0.42 \pm 0.04$ & $-0.37 \pm 0.04$ \\
225 & $2.30 \pm 0.03$ & $6.69 \pm 0.36$ & $0.54 \pm 0.02$ & $-0.30 \pm 0.02$ \\
226 & $2.29 \pm 0.03$ & $2.35 \pm 0.11$ & - & - \\
\hline & & & &
\end{tabular}


Table 8. Values for the parameters $P$ and $R_{23}$, and the metallicity derived Table 8. continued. through PT05 method for all the H II regions of NGC 628.

\begin{tabular}{|c|c|c|c|}
\hline Number & $P$ & $\log R_{23}$ & $12+\log (\mathrm{O} / \mathrm{H})$ \\
\hline 1 & 0.36 & 0.70 & $8.27 \pm 0.02$ \\
\hline 2 & 0.29 & 0.62 & $8.28 \pm 0.04$ \\
\hline 3 & 0.30 & 0.64 & $8.28 \pm 0.16$ \\
\hline 4 & - & - & - \\
\hline 5 & - & - & - \\
\hline 6 & - & - & - \\
\hline 7 & 0.41 & 0.60 & $7.98 \pm 0.22$ \\
\hline 8 & - & - & - \\
\hline 9 & 0.44 & 0.55 & $7.98 \pm 0.27$ \\
\hline 10 & - & - & - \\
\hline 11 & 0.31 & 0.25 & $8.58 \pm 0.13$ \\
\hline 12 & 0.28 & 0.67 & $7.87 \pm 0.19$ \\
\hline 13 & - & - & - \\
\hline 14 & 0.19 & 0.42 & $8.34 \pm 0.09$ \\
\hline 15 & - & - & - \\
\hline 16 & - & - & - \\
\hline 17 & 0.14 & 0.45 & $8.25 \pm 0.15$ \\
\hline 18 & 0.30 & 0.50 & $8.41 \pm 0.05$ \\
\hline 19 & - & - & - \\
\hline 20 & 0.14 & 0.46 & $8.25 \pm 0.20$ \\
\hline 21 & 0.34 & 0.38 & $8.54 \pm 0.05$ \\
\hline 22 & - & - & - \\
\hline 23 & 0.39 & 0.62 & $8.38 \pm 0.17$ \\
\hline 24 & 0.18 & 0.35 & $8.38 \pm 0.01$ \\
\hline 25 & 0.12 & 0.31 & $8.33 \pm 0.19$ \\
\hline 26 & 0.49 & 0.74 & $8.07 \pm 0.28$ \\
\hline 27 & 0.18 & 0.43 & $8.32 \pm 0.04$ \\
\hline 28 & 0.33 & 0.61 & $8.33 \pm 0.06$ \\
\hline 29 & 0.22 & 0.46 & $8.36 \pm 0.11$ \\
\hline 30 & - & - & - \\
\hline 31 & - & - & - \\
\hline 32 & - & - & - \\
\hline 33 & 0.25 & 0.37 & $8.45 \pm 0.03$ \\
\hline 34 & - & - & - \\
\hline 35 & - & - & - \\
\hline 36 & - & - & - \\
\hline 37 & - & - & - \\
\hline 38 & - & - & - \\
\hline 39 & 0.13 & 0.36 & $8.31 \pm 0.16$ \\
\hline 40 & - & - & - \\
\hline 41 & 0.28 & -0.05 & $8.66 \pm 0.26$ \\
\hline 42 & - & - & - \\
\hline 43 & 0.19 & 0.67 & $8.09 \pm 0.13$ \\
\hline 44 & 0.15 & 0.20 & $8.43 \pm 0.21$ \\
\hline 45 & - & - & - \\
\hline 46 & - & - & - \\
\hline 47 & 0.19 & 0.42 & $8.35 \pm 0.10$ \\
\hline 48 & 0.25 & 0.51 & $8.34 \pm 0.09$ \\
\hline 49 & - & - & - \\
\hline 50 & - & - & - \\
\hline 51 & 0.21 & 0.39 & $8.39 \pm 0.03$ \\
\hline 52 & 0.24 & 0.26 & $8.50 \pm 0.28$ \\
\hline 53 & - & - & - \\
\hline 54 & 0.28 & 0.71 & $8.15 \pm 0.02$ \\
\hline 55 & - & - & - \\
\hline 56 & - & - & - \\
\hline 57 & 0.21 & -0.12 & $8.62 \pm 0.16$ \\
\hline 58 & 0.16 & 0.19 & $8.45 \pm 0.03$ \\
\hline 59 & 0.07 & 0.36 & $8.22 \pm 0.09$ \\
\hline 60 & - & - & - \\
\hline 61 & - & - & - \\
\hline 62 & - & - & - \\
\hline 63 & 0.11 & -0.07 & $8.47 \pm 0.18$ \\
\hline 64 & - & - & - \\
\hline 65 & 0.24 & 0.48 & $8.36 \pm 0.04$ \\
\hline
\end{tabular}

\begin{tabular}{|c|c|c|c|}
\hline Number & $P$ & $\log R_{23}$ & $12+\log (\mathrm{O} / \mathrm{H})$ \\
\hline 66 & 0.16 & 0.20 & $8.44 \pm 0.02$ \\
\hline 67 & - & - & - \\
\hline 68 & 0.16 & 0.09 & $8.50 \pm 0.15$ \\
\hline 69 & - & - & - \\
\hline 70 & 0.23 & -0.05 & $8.62 \pm 0.26$ \\
\hline 71 & 0.16 & 0.39 & $8.33 \pm 0.18$ \\
\hline 72 & - & - & - \\
\hline 73 & - & - & - \\
\hline 74 & - & - & - \\
\hline 75 & - & - & - \\
\hline 76 & - & - & - \\
\hline 77 & - & - & - \\
\hline 78 & 0.14 & 0.03 & $8.50 \pm 0.14$ \\
\hline 79 & - & - & - \\
\hline 80 & - & - & - \\
\hline 81 & _- & - & _- \\
\hline 82 & 0.19 & 0.07 & $8.53 \pm 0.22$ \\
\hline 83 & - & - & - \\
\hline 84 & 0.25 & 0.27 & $8.51 \pm 0.01$ \\
\hline 85 & 0.07 & 0.54 & $8.04 \pm 0.08$ \\
\hline 86 & - & - & - \\
\hline 87 & - & - & - \\
\hline 88 & 0.15 & 0.47 & $8.25 \pm 0.23$ \\
\hline 89 & - & - & - \\
\hline 90 & - & - & - \\
\hline 91 & - & - & - \\
\hline 92 & - & - & - \\
\hline 93 & 0.28 & 0.08 & $8.62 \pm 0.20$ \\
\hline 94 & 0.20 & 0.57 & $8.21 \pm 0.08$ \\
\hline 95 & - & - & - \\
\hline 96 & - & - & - \\
\hline 97 & - & - & - \\
\hline 98 & - & - & - \\
\hline 99 & 0.16 & 0.53 & $8.21 \pm 0.12$ \\
\hline 100 & - & - & - \\
\hline 101 & - & - & - \\
\hline 102 & 0.28 & 0.54 & $8.35 \pm 0.13$ \\
\hline 103 & - & - & - \\
\hline 104 & 0.05 & -0.21 & $8.43 \pm 0.08$ \\
\hline 105 & - & - & - \\
\hline 106 & 0.15 & 0.25 & $8.40 \pm 0.11$ \\
\hline 107 & 0.23 & 0.65 & $8.17 \pm 0.03$ \\
\hline 108 & - & - & - \\
\hline 109 & - & - & - \\
\hline 110 & - & - & - \\
\hline 111 & - & - & - \\
\hline 112 & - & - & - \\
\hline 113 & - & - & - \\
\hline 114 & - & - & - \\
\hline 115 & - & - & - \\
\hline 116 & - & - & - \\
\hline 117 & - & - & - \\
\hline 118 & - & - & - \\
\hline 119 & - & - & - \\
\hline 120 & - & - & - \\
\hline 121 & 0.04 & 0.36 & $8.16 \pm 0.05$ \\
\hline 122 & 0.17 & 0.20 & $8.46 \pm 0.06$ \\
\hline 123 & 0.15 & 0.12 & $8.47 \pm 0.23$ \\
\hline 124 & - & - & - \\
\hline 125 & 0.32 & -0.11 & $8.71 \pm 0.30$ \\
\hline 126 & - & - & - \\
\hline 127 & - & - & - \\
\hline 128 & - & - & - \\
\hline 129 & - & - & - \\
\hline 130 & - & - & - \\
\hline 131 & 0.08 & 0.14 & $8.36 \pm 0.12$ \\
\hline
\end{tabular}


B. Cedrés et al.: Two-dimensional metallicity distribution in NGC 628 and NGC 6946

Table 8. continued.

\begin{tabular}{lccc}
\hline \hline Number & $P$ & $\log R_{23}$ & $12+\log (\mathrm{O} / \mathrm{H})$ \\
\hline 132 & - & - & - \\
133 & - & - & - \\
134 & - & - & - \\
135 & 0.24 & 0.60 & $8.25 \pm 0.16$ \\
136 & - & - & - \\
137 & - & - & - \\
138 & - & - & - \\
139 & 0.11 & -0.20 & $8.51 \pm 0.14$ \\
140 & - & - & - \\
141 & - & - & - \\
142 & 0.14 & -0.08 & $8.52 \pm 0.19$ \\
143 & - & - & - \\
144 & 0.16 & 0.26 & $8.41 \pm 0.21$ \\
145 & 0.08 & 0.36 & $8.24 \pm 0.09$ \\
146 & - & - & - \\
147 & - & - & - \\
148 & - & - & - \\
149 & - & - & - \\
150 & 0.15 & 0.59 & $8.13 \pm 0.10$ \\
151 & - & - & - \\
152 & - & - & - \\
153 & 0.12 & 0.65 & $8.00 \pm 0.16$ \\
154 & - & - & - \\
155 & 0.15 & 0.23 & $8.41 \pm 0.02$ \\
156 & 0.15 & -0.06 & $8.53 \pm 0.13$ \\
157 & - & - & - \\
158 & 0.23 & 0.10 & $8.57 \pm 0.30$ \\
159 & - & - & - \\
160 & - & - & - \\
161 & - & - & - \\
162 & 0.17 & 0.03 & $8.53 \pm 0.08$ \\
163 & - & - & - \\
164 & - & - & - \\
165 & - & - & - \\
166 & - & - & - \\
167 & 0.29 & 0.29 & $8.54 \pm 0.18$ \\
168 & 0.11 & 0.16 & $8.39 \pm 0.18$ \\
169 & 0.12 & 0.11 & $8.43 \pm 0.17$ \\
170 & - & - & - \\
\hline & & &
\end{tabular}

Table 8. continued.

\begin{tabular}{lccc}
\hline \hline Number & $P$ & $\log R_{23}$ & $12+\log (\mathrm{O} / \mathrm{H})$ \\
\hline 171 & 0.04 & 0.52 & $8.01 \pm 0.07$ \\
172 & - & - & - \\
173 & - & - & - \\
174 & - & - & - \\
175 & 0.07 & 0.42 & $8.16 \pm 0.05$ \\
176 & - & - & - \\
177 & - & - & - \\
178 & - & - & - \\
179 & - & - & - \\
180 & 0.16 & 0.67 & $8.05 \pm 0.01$ \\
181 & - & - & - \\
182 & 0.13 & 0.34 & $8.32 \pm 0.06$ \\
183 & - & - & - \\
184 & 0.13 & 0.44 & $8.24 \pm 0.15$ \\
185 & - & - & - \\
186 & - & - & - \\
187 & 0.43 & -0.05 & $8.77 \pm 0.06$ \\
188 & 0.15 & 0.28 & $8.39 \pm 0.06$ \\
189 & - & - & - \\
190 & 0.16 & 0.24 & $8.43 \pm 0.22$ \\
191 & 0.12 & 0.18 & $8.40 \pm 0.13$ \\
192 & 0.06 & 0.36 & $8.19 \pm 0.07$ \\
193 & 0.14 & 0.27 & $8.37 \pm 0.04$ \\
194 & 0.18 & 0.34 & $8.39 \pm 0.26$ \\
195 & 0.21 & 0.43 & $8.36 \pm 0.25$ \\
196 & 0.21 & 0.51 & $8.29 \pm 0.03$ \\
197 & 0.15 & 0.55 & $8.16 \pm 0.22$ \\
198 & 0.17 & 0.73 & $7.97 \pm 0.06$ \\
199 & - & - & - \\
200 & 0.21 & 0.30 & $8.46 \pm 0.03$ \\
201 & 0.10 & 0.06 & $8.43 \pm 0.13$ \\
202 & 0.14 & 0.51 & $8.19 \pm 0.01$ \\
203 & 0.10 & 0.44 & $8.20 \pm 0.05$ \\
204 & 0.30 & 0.67 & $8.24 \pm 0.10$ \\
205 & 0.22 & 0.57 & $8.25 \pm 0.01$ \\
206 & 0.14 & 0.59 & $8.11 \pm 0.05$ \\
207 & 0.21 & 0.52 & $8.28 \pm 0.05$ \\
208 & 0.20 & 0.39 & $8.38 \pm 0.25$ \\
209 & - & - & - \\
\hline & & &
\end{tabular}


Table 9. Values for the parameters $P$ and $R_{23}$, and the metallicity derived through PT05 method for all the H II regions of NGC 6946.

\begin{tabular}{|c|c|c|c|}
\hline Number & $P$ & $\log R_{23}$ & $12+\log (\mathrm{O} / \mathrm{H})$ \\
\hline 1 & - & - & - \\
\hline 2 & 0.10 & 0.58 & $8.05 \pm 0.13$ \\
\hline 3 & 0.22 & 0.56 & $8.27 \pm 0.06$ \\
\hline 4 & - & - & - \\
\hline 5 & 0.09 & 0.57 & $8.05 \pm 0.08$ \\
\hline 6 & 0.24 & 0.61 & $8.22 \pm 0.05$ \\
\hline 7 & 0.10 & 0.42 & $8.22 \pm 0.05$ \\
\hline 8 & 0.12 & 0.39 & $8.27 \pm 0.08$ \\
\hline 9 & 0.07 & 0.53 & $8.06 \pm 0.06$ \\
\hline 10 & - & - & - \\
\hline 11 & 0.27 & 0.61 & $8.26 \pm 0.20$ \\
\hline 12 & 0.44 & 0.76 & $8.27 \pm 0.16$ \\
\hline 13 & - & - & - \\
\hline 14 & 0.18 & 0.37 & $8.37 \pm 0.07$ \\
\hline 15 & 0.21 & 0.46 & $8.34 \pm 0.09$ \\
\hline 16 & 0.19 & 0.77 & - \\
\hline 17 & - & - & - \\
\hline 18 & 0.31 & 0.45 & $8.46 \pm 0.06$ \\
\hline 19 & - & - & - \\
\hline 20 & - & - & - \\
\hline 21 & 0.18 & 0.47 & $8.28 \pm 0.06$ \\
\hline 22 & 0.16 & 0.56 & $8.17 \pm 0.08$ \\
\hline 23 & - & - & - \\
\hline 24 & 0.15 & 0.64 & $8.05 \pm 0.10$ \\
\hline 25 & - & - & - \\
\hline 26 & 0.13 & 0.45 & $8.23 \pm 0.05$ \\
\hline 27 & 0.19 & 0.32 & $8.41 \pm 0.17$ \\
\hline 28 & 0.22 & 0.29 & $8.47 \pm 0.26$ \\
\hline 29 & - & - & - \\
\hline 30 & - & - & - \\
\hline 31 & - & - & - \\
\hline 32 & - & - & - \\
\hline 33 & 0.14 & 0.26 & - \\
\hline 34 & 0.18 & 0.20 & $8.47 \pm 0.03$ \\
\hline 35 & - & - & - \\
\hline 36 & 0.07 & 0.46 & $8.13 \pm 0.05$ \\
\hline 37 & - & - & - \\
\hline 38 & 0.17 & 0.30 & $8.40 \pm 0.04$ \\
\hline 39 & - & - & - \\
\hline 40 & - & - & - \\
\hline 41 & - & - & - \\
\hline 42 & - & - & - \\
\hline 43 & - & - & - \\
\hline 44 & 0.11 & 0.21 & $8.37 \pm 0.03$ \\
\hline 45 & - & - & - \\
\hline 46 & - & - & - \\
\hline 47 & - & - & - \\
\hline 48 & 0.15 & 0.15 & $8.45 \pm 0.03$ \\
\hline 49 & - & - & - \\
\hline 50 & - & - & - \\
\hline 51 & - & - & - \\
\hline 52 & - & - & - \\
\hline 53 & 0.18 & 0.36 & $8.38 \pm 0.04$ \\
\hline 54 & - & - & - \\
\hline 55 & 0.13 & 0.15 & $8.43 \pm 0.04$ \\
\hline 56 & - & - & - \\
\hline 57 & 0.30 & 0.43 & $8.47 \pm 0.05$ \\
\hline 58 & 0.10 & 0.26 & $8.33 \pm 0.06$ \\
\hline 59 & - & - & - \\
\hline 60 & - & - & - \\
\hline 61 & - & - & - \\
\hline 62 & - & - & - \\
\hline 63 & 0.20 & 0.09 & $8.54 \pm 0.03$ \\
\hline 64 & 0.13 & 0.25 & $8.38 \pm 0.19$ \\
\hline
\end{tabular}

Table 9. Values for the parameters $P$ and $R_{23}$, and the metallicity derived through PT05 method for all the H II regions of NGC 6946.

\begin{tabular}{|c|c|c|c|}
\hline Number & $P$ & $\log R_{23}$ & $12+\log (\mathrm{O} / \mathrm{H})$ \\
\hline 65 & 0.20 & 0.32 & \\
\hline 66 & 0.29 & 0.07 & $8.64 \pm 0.18$ \\
\hline 67 & - & - & - \\
\hline 68 & - & - & - \\
\hline 69 & - & - & - \\
\hline 70 & - & - & - \\
\hline 71 & - & _- & - \\
\hline 72 & 0.11 & 0.64 & $8.00 \pm 0.20$ \\
\hline 73 & - & - & - \\
\hline 74 & - & - & - \\
\hline 75 & - & - & - \\
\hline 76 & - & - & - \\
\hline 77 & - & - & - \\
\hline 78 & 0.20 & 0.14 & $8.52 \pm 0.04$ \\
\hline 79 & - & - & - \\
\hline 80 & 0.16 & 0.09 & $8.49 \pm 0.03$ \\
\hline 81 & - & - & - \\
\hline 82 & - & - & - \\
\hline 83 & - & - & - \\
\hline 84 & - & - & - \\
\hline 85 & 0.36 & 0.21 & $8.64 \pm 0.26$ \\
\hline 86 & - & - & - \\
\hline 87 & - & - & - \\
\hline 88 & - & - & - \\
\hline 89 & - & - & - \\
\hline 90 & - & - & - \\
\hline 91 & - & - & - \\
\hline 92 & _- & _- & _- \\
\hline 93 & - & - & - \\
\hline 94 & - & - & - \\
\hline 95 & - & - & - \\
\hline 96 & _- & _- & - \\
\hline 97 & - & - & - \\
\hline 98 & - & - & - \\
\hline 99 & - & _- & _- \\
\hline 100 & - & - & - \\
\hline 101 & - & - & - \\
\hline 102 & - & - & - \\
\hline 103 & - & - & - \\
\hline 104 & - & - & - \\
\hline 105 & - & - & - \\
\hline 106 & - & - & - \\
\hline 107 & - & - & - \\
\hline 108 & - & - & - \\
\hline 109 & - & - & - \\
\hline 110 & - & - & - \\
\hline 111 & - & - & - \\
\hline 112 & - & - & - \\
\hline 113 & 0.11 & 0.09 & $8.43 \pm 0.02$ \\
\hline 114 & - & - & - \\
\hline 115 & - & - & - \\
\hline 116 & - & - & - \\
\hline 117 & 0.14 & 0.28 & $8.37 \pm 0.11$ \\
\hline 118 & - & - & - \\
\hline 119 & - & - & - \\
\hline 120 & - & - & - \\
\hline 121 & 0.21 & 0.04 & - \\
\hline 122 & - & - & - \\
\hline 123 & - & - & - \\
\hline 124 & - & _- & - \\
\hline 125 & - & - & - \\
\hline 126 & - & - & - \\
\hline 127 & - & - & - \\
\hline 128 & _- & - & - \\
\hline
\end{tabular}


B. Cedrés et al.: Two-dimensional metallicity distribution in NGC 628 and NGC 6946

Table 9. continued.

\begin{tabular}{|c|c|c|c|}
\hline Number & $P$ & $\log R_{23}$ & $12+\log (\mathrm{O} / \mathrm{H})$ \\
\hline 129 & 0.15 & 0.16 & $8.45 \pm 0.27$ \\
\hline 130 & 0.18 & 0.21 & $8.47 \pm 0.02$ \\
\hline 131 & - & - & - \\
\hline 132 & - & - & - \\
\hline 133 & - & - & - \\
\hline 134 & 0.11 & 0.23 & $8.36 \pm 0.05$ \\
\hline 135 & 0.11 & 0.38 & $8.26 \pm 0.03$ \\
\hline 136 & 0.12 & 0.46 & $8.20 \pm 0.18$ \\
\hline 137 & - & - & - \\
\hline 138 & 0.19 & 0.20 & $8.48 \pm 0.03$ \\
\hline 139 & - & - & - \\
\hline 140 & - & - & - \\
\hline 141 & - & - & - \\
\hline 142 & 0.12 & 0.01 & $8.47 \pm 0.19$ \\
\hline 143 & - & - & - \\
\hline 144 & - & - & - \\
\hline 145 & - & - & - \\
\hline 146 & 0.17 & -0.00 & $8.53 \pm 0.02$ \\
\hline 147 & - & - & - \\
\hline 148 & 0.21 & 0.33 & - \\
\hline 149 & - & - & - \\
\hline 150 & - & - & - \\
\hline 151 & 0.17 & 0.28 & - \\
\hline 152 & 0.31 & 0.55 & $8.37 \pm 0.07$ \\
\hline 153 & 0.11 & 0.73 & $7.87 \pm 0.07$ \\
\hline 154 & 0.17 & 0.30 & $8.40 \pm 0.04$ \\
\hline 155 & - & - & - \\
\hline 156 & - & - & - \\
\hline 157 & 0.14 & 0.47 & $8.23 \pm 0.07$ \\
\hline 158 & - & - & - \\
\hline 159 & 0.15 & 0.24 & $8.41 \pm 0.04$ \\
\hline 160 & - & - & - \\
\hline 161 & - & - & - \\
\hline 162 & - & - & - \\
\hline 163 & - & - & - \\
\hline 164 & - & - & - \\
\hline 165 & - & - & - \\
\hline 166 & - & - & - \\
\hline 167 & - & - & - \\
\hline 168 & 0.12 & 0.25 & $8.36 \pm 0.03$ \\
\hline 169 & - & - & - \\
\hline 170 & - & - & - \\
\hline 171 & - & - & - \\
\hline 172 & - & - & - \\
\hline 173 & 0.12 & 0.43 & $8.23 \pm 0.10$ \\
\hline 174 & - & - & - \\
\hline 175 & - & - & - \\
\hline 176 & 0.22 & 0.28 & $8.47 \pm 0.03$ \\
\hline 177 & 0.27 & 0.20 & $8.57 \pm 0.07$ \\
\hline
\end{tabular}

Table 9. continued.

\begin{tabular}{|c|c|c|c|}
\hline Number & $P$ & $\log R_{23}$ & $12+\log (\mathrm{O} / \mathrm{H})$ \\
\hline 178 & - & - & - \\
\hline 179 & _- & - & - \\
\hline 180 & 0.17 & 0.14 & $8.49 \pm 0.04$ \\
\hline 181 & - & - & - \\
\hline 182 & - & - & - \\
\hline 183 & - & - & - \\
\hline 184 & - & - & - \\
\hline 185 & _- & _- & _- \\
\hline 186 & _- & - & _- \\
\hline 187 & 0.22 & 0.24 & $8.49 \pm 0.05$ \\
\hline 188 & 0.46 & 0.45 & $8.59 \pm 0.04$ \\
\hline 189 & - & - & - \\
\hline 190 & 0.17 & 0.21 & $8.46 \pm 0.03$ \\
\hline 191 & - & - & - \\
\hline 192 & - & - & - \\
\hline 193 & - & - & _- \\
\hline 194 & - & - & - \\
\hline 195 & 0.06 & 0.64 & $7.92 \pm 0.06$ \\
\hline 196 & - & - & - \\
\hline 197 & - & - & - \\
\hline 198 & - & - & - \\
\hline 199 & 0.23 & 0.29 & $8.47 \pm 0.05$ \\
\hline 200 & 0.28 & 0.46 & $8.42 \pm 0.41$ \\
\hline 201 & 0.27 & 0.06 & $8.62 \pm 0.09$ \\
\hline 202 & - & - & - \\
\hline 203 & - & - & - \\
\hline 204 & - & - & - \\
\hline 205 & 0.21 & 0.23 & $8.49 \pm 0.09$ \\
\hline 206 & 0.14 & 0.27 & $8.39 \pm 0.04$ \\
\hline 207 & 0.09 & 0.69 & $7.89 \pm 0.05$ \\
\hline 208 & - & - & - \\
\hline 209 & - & - & - \\
\hline 210 & 0.13 & 0.43 & $8.25 \pm 0.03$ \\
\hline 211 & 0.14 & 0.43 & $8.27 \pm 0.03$ \\
\hline 212 & - & - & - \\
\hline 213 & 0.13 & 0.32 & $8.33 \pm 0.05$ \\
\hline 214 & 0.20 & 0.43 & $8.35 \pm 0.03$ \\
\hline 215 & - & - & - \\
\hline 216 & 0.13 & 0.46 & $8.22 \pm 0.05$ \\
\hline 217 & - & - & - \\
\hline 218 & 0.15 & 0.28 & $8.38 \pm 0.19$ \\
\hline 219 & - & - & - \\
\hline 220 & - & - & - \\
\hline 221 & 0.10 & 0.41 & $8.22 \pm 0.13$ \\
\hline 222 & - & - & - \\
\hline 223 & 0.17 & 0.49 & $8.26 \pm 0.03$ \\
\hline 224 & 0.14 & 0.49 & $8.21 \pm 0.06$ \\
\hline 225 & 0.13 & 0.60 & $8.08 \pm 0.03$ \\
\hline 226 & - & - & - \\
\hline
\end{tabular}

